\title{
On the monotone stability approach to BSDEs with jumps: Extensions, concrete criteria and examples *
}

\author{
Dirk Becherer ${ }^{\dagger}$ \\ Martin Büttner \\ Klebert Kentia $\ddagger$
}

November 21, 2019

\begin{abstract}
We show a concise extension of the monotone stability approach to backward stochastic differential equations (BSDEs) that are jointly driven by a Brownian motion and a random measure of jumps, which could be of infinite activity with a non-deterministic and timeinhomogeneous compensator. The BSDE generator function can be non-convex and needs not satisfy global Lipschitz conditions in the jump integrand. We contribute concrete sufficient criteria, that are easy to verify, for results on existence and uniqueness of bounded solutions to BSDEs with jumps, and on comparison and a-priori $L^{\infty}$-bounds. Several examples and counter examples are discussed to shed light on the scope and applicability of different assumptions, and we provide an overview of major applications in finance and optimal control.
\end{abstract}

Keywords: Backward stochastic differential equations, random measures, monotone stability, Lévy processes, step processes, utility maximization, entropic risk measure, good deal valuation bounds MSC2010: 60G57, 60H20, 93E20, 60G51, 91G80

\section{Introduction}

We study bounded solutions $(Y, Z, U)$ to backward stochastic differential equations with jumps

$$
Y_{t}=\xi+\int_{t}^{T} f_{s}\left(Y_{s-}, Z_{s}, U_{s}\right) \mathrm{d} s-\int_{t}^{T} Z_{s} \mathrm{~d} B_{s}-\int_{t}^{T} \int_{E} U_{s}(e) \widetilde{\mu}(\mathrm{d} s, \mathrm{~d} e),
$$

which are jointly driven by a Brownian motion $B$ and a compensated random measure $\widetilde{\mu}=\mu-\nu^{\mathbb{P}}$ of some integer-valued random measure $\mu$ on a probability space $(\Omega, \mathcal{F}, \mathbb{P})$. This is an extension of the classical BSDE theory on Wiener space towards BSDEs which involve jumps (JBSDEs), that are driven by the compensated random measure $\widetilde{\mu}$, and do evolve on non-Brownian filtrations. Such JBSDEs do involve an additional stochastic integral with respect to the compensated jump measure $\widetilde{\mu}$ whose integrand $U$, differently from $Z$, typically takes values in an infinite dimensional function space instead of an Euclidean space.

Comparison theorems for BSDEs with jumps require more delicate technical conditions than in the Brownian case, see [BBP97, Roy06, CE10]. The starting point for our article will be a slight generalization of the seminal $\left(\mathbf{A}_{\gamma}\right)$-condition for comparison due to [Roy06]. Our first contribution are extensions of comparison, existence and uniqueness results for bounded solutions of JBSDEs to the case of infinite jump activity for a family (2.6) of generators, that do not need to be Lipschitz in the $U$-argument. This shows how the monotone stability approach to BSDEs with jumps, pioneered by [Mor09] for one particular generator, permits for a concise proof in a setting, that may be of particular appeal in a pure jump case without a Brownian motion, see Corollary 4.12. While the strong approximation step for this ansatz is usually laborious, we present a compact

\footnotetext{
*For the final publication, please refer to Frontiers in Stochastic Analysis - BSDEs, SPDEs and their Applications, Springer, 2019, DOI 10.1007/978-3-030-22285-7_1

†Institut für Mathematik, Humboldt-Universität zu Berlin, Unter den Linden 6, D-10099 Berlin, Germany.

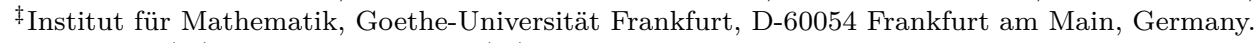
Emails: kentia (at) aims.ac.za, becherer (at) math.hu-berlin.de.
} 
proof with a $\mathcal{S}^{1}$-closedness argument and more generality of the generator in the $U$-argument for infinite activity of jumps. To be useful towards applications, our second contribution are sufficient concrete criteria for comparison and wellposedness that are comparably easy to verify in actual examples, because they are formulated in terms of concrete properties for generator functions $f$ from a given family (2.6) w.r.t. to basically Euclidean arguments, instead of assuming inequalities to hold for rather abstract random processes or fields. This is the main thrust for the sufficient conditions of the comparison results in Section 3 (see Theorem 3.9 and Proposition 3.11, compared to Proposition 3.1 or the result by [Roy06] and respective enhancements [QS13, KP16, Yao17]) and of the wellposedness Theorem 4.13 (in comparison to Theorem 4.11, whose conditions are more general but more abstract). A third contribution are the many examples and applications which illustrate the scope and applicability of our results and of the, often technical, assumptions that are needed for JBSDE results in the literature. Indeed, the range of the imposed combinations of several technical assumptions is often not immediately clear. We believe that more discussion of examples and counter examples may help to shed light on the scope and the differences of some assumptions prevailing in the literature, and might also caution against possible pitfalls.

The approach in this paper can be described in more detail as follows: The comparison results will provide basic a-priori estimates on the $L^{\infty}$-norm for the $Y$-component of the JBSDE solution. This step enables a quick intermediate result on existence and uniqueness for JBSDEs with finite jump activity. To advance from here to infinite activity, we approximate the generator $f$ by a monotone sequence of generators for which solutions do exist, extending the monotone stability approach from [Kob00] and (for a particular JBSDE) [Mor09]. For the present paper, the compensator $\nu(\omega, \mathrm{d} t, \mathrm{~d} e)$ of $\mu(\omega, \mathrm{d} t, \mathrm{~d} e)$ can be stochastic and does not need to be a product measure like $\lambda(\mathrm{d} e) \otimes \mathrm{d} t$, as it would be natural e.g. in a Lévy-process setting, but it is allowed to be inhomogeneous in that it can vary predictably with $(\omega, t)$. In this sense, $\nu$ is only assumed to be absolutely continuous to some reference product measure $\lambda \otimes \mathrm{d} t$ with $\lambda$ being $\sigma$-finite, see equation (2.1). Such appears useful, but requires some care in the specification of generator properties in Section 2. For the filtration we assume that $\widetilde{\mu}$ jointly with $B$ (or alone) satisfies the property of weak predictable representation for martingales, see (2.2). As explained in Example 2.1, such setup permits for a range of stochastic dependencies between $B$ and $\widetilde{\mu}$, which appear useful for modeling of applications, and encompasses many interesting driving noises for jumps in BSDEs; This includes Lévy processes, Poisson random measures, marked point processes, (semi-)Markov chains or much more general step processes, connecting to a wide range of literature, e.g. [CE10, CE15, CFJ16, GL16, GS16a, GS16b, BC17].

The literature on BSDE started with the classical study [PP90] of square integrable solutions to BSDEs driven solely by Brownian motion $B$ under global Lipschitz assumptions. One important extension concerns generators $f$ which are non-Lipschitz but have quadratic growth in $Z$, for which [Kob00] derived bounded solutions by pioneering a monotone stability approach, and [Tev08] by a fixed point approach. Square integrable solutions under global Lipschitz conditions for BSDEs with jumps from a Poisson random measures are first studied by [TL94, BBP97]. There is a lot of development in JBSDE theory recently. See for instance [Ban15, PPS16, KP16, KP17, EFO17] for results under global Lipschitz conditions on the generator with respect to on $(Z, U)$. In the context of non-Lipschitz generators that are quadratic (also in $Z$, with exponential growth in $U$ ), JBSDEs have been studied to our knowledge at first by [Mor09] using a monotone stability approach for a specific generator that is related to exponential utility, by [EMN16] using a quadratic-exponential semimartingale approach from [BEK13], and by [LS14] or [KTPZ15] with again different approaches, relying on duality methods or, respectively, the fixed-point idea of [Tev08] for quadratic BSDEs. For extensive surveys of the active literature with more references, let us refer to [KP16, Yao17], who contribute results on $L^{p}$-solutions for generators, being monotone in the $Y$-component, that are very general in many aspects. Their assumptions on the filtrations or generator's dependence on $(Y, Z)$ are for instance more general than ours. But the present paper still contributes on other aspects, noted above. For instance, [Yao17] assumes finite activity of jumps and a Lipschitz continuity in $U$. More relations to some other related literature are being explained in many examples throughout the paper, see e.g. in Section 5. Moreover, it is fair to say that results in the JBSDE literature often involve combinations of many technical assumptions; To understand the scope, applicability and differences of those assumptions, it appears helpful to discuss concrete examples and applications.

The paper is organized as follows. Section 2 introduces the setting and mathematical background. In Sections 3-4, we prove comparison results and show existence as well as uniqueness for bounded 
solutions to JBSDEs, both for finite and infinite activity of jumps. Last but not least, Section 5 surveys key applications of JBSDEs in finance. We discuss several examples to shed light on the scope of the results and of the underlying technical assumptions, and connections to the literature.

\section{Preliminaries}

This section presents the technical framework, sets notations and discusses key conditions. First we recall essential facts on stochastic integration w.r.t. random measures and on bounded solutions for Backward SDEs which are driven jointly by Brownian motions and a compensated random measure. For notions from stochastic analysis not explained here we refer to [JS03, HWY92].

Inequalities between measurable functions are understood almost everywhere w.r.t. an appropriate reference measure, typically $\mathbb{P}$ or $\mathbb{P} \otimes \mathrm{d} t$. Let $T<\infty$ be a finite time horizon and $\left(\Omega, \mathcal{F},\left(\mathcal{F}_{t}\right)_{0 \leq t \leq T}, \mathbb{P}\right)$ a filtered probability space with a filtration $\mathbb{F}=\left(\mathcal{F}_{t}\right)_{0 \leq t \leq T}$ satisfying the usual conditions of right continuity and completeness, assuming $\mathcal{F}_{T}=\mathcal{F}$ and $\mathcal{F}_{0}$ being trivial (under $\mathbb{P})$; Thus we can and do take all semimartingales to have right continuous paths with left limits, so-called càdlàg paths. Expectations (under $\mathbb{P}$ ) are denoted by $\mathbb{E}=\mathbb{E}_{\mathbb{P}}$. We will denote by $\mathbf{A}^{T}$ the transpose of a matrix $\mathbf{A}$ and simply write $\boldsymbol{x} \boldsymbol{y}:=\boldsymbol{x}^{T} \boldsymbol{y}$ for the scalar product for two vectors $\boldsymbol{x}, \boldsymbol{y}$ of same dimensionality. Let $H$ be a separable Hilbert space and denote by $\mathcal{B}(E)$ the Borel $\sigma$-field of $E:=H \backslash\{0\}$, e.g. $H=\mathbb{R}^{l}, l \in \mathbb{N}$ or $H=\ell^{2} \subset \mathbb{R}^{\mathbb{N}}$. Then $(E, \mathcal{B}(E))$ is a standard Borel space. In addition, let $B$ be a $d$-dimensional Brownian motion. Stochastic integrals of a vector valued predictable process $Z$ w.r.t. a semimartingale $X$, e.g. $X=B$, of the same dimensionality are scalar valued semimartingales starting at zero and denoted by $\int_{(0, t]} Z \mathrm{~d} X=\int_{0}^{t} Z \mathrm{~d} X=Z \cdot X_{t}$ for $t \in[0, T]$. The predictable $\sigma$-field on $\Omega \times[0, T]$ (w.r.t. $\left(\mathcal{F}_{t}\right)_{0 \leq t \leq T}$ ) is denoted by $\mathcal{P}$ and $\widetilde{\mathcal{P}}:=\mathcal{P} \otimes \mathcal{B}(E)$ is the respective $\sigma$-field on $\widetilde{\Omega}:=\Omega \times[0, T] \times E$.

Let $\mu$ be an integer-valued random measure with compensator $\nu=\nu^{\mathbb{P}}$ (under $\mathbb{P}$ ) which is taken to be absolutely continuous to $\lambda \otimes \mathrm{d} t$ for a $\sigma$-finite measure $\lambda$ on $(E, \mathcal{B}(E))$ satisfying $\int_{E} 1 \wedge|e|^{2} \lambda(\mathrm{d} e)<\infty$ with some $\widetilde{\mathcal{P}}$-measurable, bounded and non-negative density $\zeta$, such that

$$
\nu(\mathrm{d} t, \mathrm{~d} e)=\zeta(t, e) \lambda(\mathrm{d} e) \mathrm{d} t=\zeta_{t} \mathrm{~d} \lambda \mathrm{d} t,
$$

with $0 \leq \zeta(t, e) \leq c_{\nu} \mathbb{P} \otimes \lambda \otimes \mathrm{d} t$-a.e. for some constant $c_{\nu}>0$. Note that $L^{2}(\lambda)$ and $L^{2}\left(\zeta_{t} \mathrm{~d} \lambda\right)$ are separable Hilbert spaces since $\lambda$ (and $\lambda_{t}:=\zeta_{t} \mathrm{~d} \lambda$ ) is $\sigma$-finite and $\mathcal{B}(E)$ is finitely generated. Since the density $\zeta$ can vary with $(\omega, t)$, the compensator $\nu$ can be time-inhomogeneous and stochastic. Such permits for a richer dependence structure for $(B, \widetilde{\mu})$; For instance, the intensity and distribution of jump heights could vary according to some diffusion process. Yet, it also brings a few technical complications, e.g. function-valued integrand processes $U$ from $\mathcal{L}^{2}(\widetilde{\mu})$ (as defined below) for the JBSDE need not take values in one given $L^{2}$-space (for a.e. $(\omega, t)$ ), like e.g. $L^{2}(\lambda)$ if $\zeta \equiv 1$, and the specifications of the domain and of the measurability for the generator functions should take account of such.

For stochastic integration w.r.t. $\widetilde{\mu}$ and $B$ we define sets of $\mathbb{R}$-valued processes

$$
\begin{aligned}
\mathcal{S}^{p} & :=\mathcal{S}^{p}(\mathbb{P}):=\left\{Y \text { càdlàg }:|Y|_{p}:=\left\|\sup _{0 \leq t \leq T}\left|Y_{t}\right|\right\|_{L^{p}(\mathbb{P})}<\infty\right\} \text { for } p \in[1, \infty], \\
\mathcal{L}^{2}(\widetilde{\mu}) & :=\left\{U \widetilde{\mathcal{P}} \text {-measurable }:\|U\|_{\mathcal{L}^{2}(\widetilde{\mu})}^{2}:=\mathbb{E}\left(\int_{0}^{T} \int_{E}\left|U_{s}(e)\right|^{2} \nu(\mathrm{d} s, \mathrm{~d} e)\right)<\infty\right\},
\end{aligned}
$$

and the set of $\mathbb{R}^{d}$-valued processes

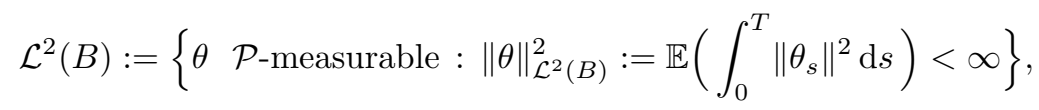

where $\widetilde{\mu}=\widetilde{\mu}^{\mathbb{P}}=\mu-\nu$ denotes the compensated measure of $\mu$ (under $\mathbb{P}$ ). Recall that for any predictable function $U, \mathbb{E}\left(|U| * \mu_{T}\right)=\mathbb{E}\left(|U| * \nu_{T}\right)$ by the definition of a compensator. If $\left(|U|^{2} * \mu\right)^{1 / 2}$ is locally integrable, then $U$ is integrable w.r.t. $\widetilde{\mu}$, and $U * \widetilde{\mu}$ is defined as the purely discontinuous local martingale with jump process $\left(\int_{E} U_{t}(e) \mu(\{t\}, \mathrm{d} e)\right)_{t}$ by [JS03, Def.II.1.27] noting that $\nu$ is absolutely continuous to $\lambda \otimes \mathrm{d} t$. For $Z \in \mathcal{L}^{2}(B)$ and $U \in \mathcal{L}^{2}(\widetilde{\mu})$ we recall 
that $Z \cdot B$ and $U * \widetilde{\mu}=\left(U * \widetilde{\mu}_{t}\right)_{0 \leq t \leq T}$ with $U * \widetilde{\mu}_{t}=\int_{0}^{t} \int_{E} U_{s}(e) \widetilde{\mu}(\mathrm{d} s, \mathrm{~d} e)$ are square integrable martingales by [JS03, Thm.II.1.33]. For $Z, Z^{\prime} \in \mathcal{L}^{2}(B)$ and $U, U^{\prime} \in \mathcal{L}^{2}(\widetilde{\mu})$ we have for the predictable quadratic covariations that $\left\langle U * \widetilde{\mu}, U^{\prime} * \widetilde{\mu}\right\rangle_{t}=\int_{0}^{t} \int_{E} U_{s}(e) U_{s}^{\prime}(e) \nu(\mathrm{d} s, \mathrm{~d} e)$ by [JS03, Thm.II.1.33], $\left\langle\int Z \mathrm{~d} B, \int Z^{\prime} \mathrm{d} B\right\rangle_{t}=\int_{0}^{t} Z_{s}^{T} Z_{s}^{\prime} \mathrm{d} s$ and $\left\langle\int Z \mathrm{~d} B, U * \widetilde{\mu}\right\rangle_{t}=0$ by [JS03, Thm.I.4.2].

We denote the space of square integrable martingales by $\mathcal{M}^{2}$ and its norm by $\|\cdot\|_{\mathcal{M}^{2}}$ with $\|M\|_{\mathcal{M}^{2}}=$ $\mathbb{E}\left(M_{T}^{2}\right)^{1 / 2}$. We recall $[\mathrm{HWY} 92$, Thm.10.9.4] that the subspace of $\mathrm{BMO}(\mathbb{P})$-martingales $\mathrm{BMO}(\mathbb{P})$ contains any square integrable martingale $M$ with uniformly bounded jumps and bounded conditional expectations for increments of the quadratic variation process:

$$
\sup _{0 \leq t \leq T}\left\|\mathbb{E}\left(\left(M_{T}-M_{t}\right)^{2} \mid \mathcal{F}_{t}\right)\right\|_{L^{\infty}(\mathbb{P})}=\sup _{0 \leq t \leq T}\left\|\mathbb{E}\left(\langle M\rangle_{T}-\langle M\rangle_{t} \mid \mathcal{F}_{t}\right)\right\|_{L^{\infty}(\mathbb{P})} \leq \text { const }<\infty .
$$

We will assume that the continuous martingale $B$ and the compensated measure $\widetilde{\mu}$ of an integervalued random measure $\mu$ (or $\widetilde{\mu}$ alone, see Example 2.1.1 and Corollary 4.12 with trivial $B=0$ ) jointly have the weak predictable representation property (weak PRP) w.r.t. the filtration $\left(\mathcal{F}_{t}\right)_{0 \leq t \leq T}$, in that every square integrable martingale $M$ has a (unique) representation, i.e.

$$
\text { for all } M \in \mathcal{M}^{2} \text { there exists } Z, U \text { such that } M=M_{0}+\int Z \mathrm{~d} B+U * \widetilde{\mu},
$$

with (unique) $Z \in \mathcal{L}^{2}(B)$ and $U \in \mathcal{L}^{2}(\widetilde{\mu})$. Let us note that in the literature [JS03, III.\$4c] or [HWY92, XIII.§2] the weak representation property is defined as a decomposition like (2.2) for any local martingale $M$ with integrands $Z, U$ being integrable in the sense of local martingales. Such clearly implies our formulation above. Indeed, for a (locally) square integrable martingale $M$ in such a decomposition both integrands must be at least locally square integrable and $\langle M\rangle=$ $\int|Z|^{2} \mathrm{~d} t+|U|^{2} * \nu$ by strong orthogonality of the stochastic integrals. Then $E\left[\langle M\rangle_{T}\right]<\infty$ implies that $Z, U$ are in the respective $\mathcal{L}^{2}$-spaces. We exemplify how (2.2) connects with a wide literature.

Example 2.1. The weak predictable representation property (2.2) holds in the cases below. Cases 1.-4. are well known from classical theory [HWY92], see [Bec06, Example 2.1] for details.

1. Let $X$ be a Lévy process with $X_{0}=0$ and predictable characteristics $(\alpha, \beta, \nu)$ (under $\left.\mathbb{P}\right)$. Then the continuous martingale part $X^{c}$ (rescaled to a Brownian motion if $\beta \neq 0$, or being trivial if $\beta=0$ ) and the compensated jump measure $\widetilde{\mu}^{X}=\mu^{X}-\nu$ of $X$ have the weak PRP w.r.t. the usual filtration $\mathbb{F}^{X}$ generated by $X$. An example for a Lévy process of infinite activity is the Gamma process. One can add that weak PRP even holds in the sense of Thm III.4.34 from [JS03] for the more general class of PII-processes with independent increments. This class encompasses the more familiar Lévy processes without requiring time-homogeneity or stochastic continuity.

2. Assume that $B$ and $\widetilde{\mu}$ satisfy (2.2) under $\mathbb{P}$. Let $\mathbb{P}^{\prime}$ be an equivalent probability measure with density process $Z$. Then the Brownian motion $B^{\prime}:=B-\int\left(Z_{-}\right)^{-1} \mathrm{~d}\langle Z, B\rangle$ and $\widetilde{\mu}^{\prime}:=\mu-\nu^{\mathbb{P}^{\prime}}$ have the weak PRP (2.2) also w.r.t. $\mathbb{P}^{\prime}$ under the same filtration. This offers plenty of scope to construct examples where $W$ and $\widetilde{\mu}$ are not independent, based on other examples.

3. Let $B$ be a Brownian motion independent of a step process $X$ (in the sense of [HWY92, Ch. 11]). Then $B$ and $\tilde{\mu}$, the compensated measure of the jump measure $\mu^{X}$ of $X$, have the weak PRP w.r.t. the usual filtration generated by $X$ and $B$. An example for a step process is a multivariate (non-explosive) point process, as appearing in [CFJ16].

4. A (semi-)Markov chain X, possibly time-inhomogeneous, is a step process. Thus weak PRP (2.2) holds for a filtration generated by a Brownian motion and an independent Markov chain, relating later results to literature [CE10, CF14, BC17] on BSDEs driven by compensated random measures of the respective pure-jump (semi-)Markov processes. Markov chains $X$ on countable state spaces can be chosen [CE10] to take values in the set of unit vectors $\left\{e_{i}: i \in \mathbb{N}\right\}$ of the sequence space $\ell^{2} \subset \mathbb{R}^{\mathbb{N}}$, with jumps $\Delta X$ taking values $e_{i}-e_{j}, i, j \in \mathbb{N}$.

5. The pure jump martingale $U * \widetilde{\mu}$ (for $U \in \mathcal{L}^{2}(\widetilde{\mu})$ ) may be written as a series of mutually orthogonal martingales. More precisely, assume that the compensator coincides with the product 
measure $\lambda \otimes \mathrm{d} t$, i.e. $\zeta=1$. Let $\left(u^{n}\right)_{n \in \mathbb{N}}$ be an orthonormal basis (ONB) of the separable Hilbert space $L^{2}(\lambda)$ with scalar product $\langle u, v\rangle:=\int_{E} u(e) v(e) \lambda(\operatorname{de})$. Let $U_{t}=\sum_{n \in \mathbb{N}}\left\langle U_{t}, u^{n}\right\rangle u^{n}$ be the basis expansion of $U_{t}$ for $U \in \mathcal{L}^{2}(\widetilde{\mu}), t \leq T$. Then it holds (in $\left.\mathcal{M}^{2}\right)$

$$
U * \widetilde{\mu}=\sum_{n \in \mathbb{N}} \int_{0}^{\cdot}\left\langle U_{t}, u^{n}\right\rangle \int_{E} u^{n}(e) \widetilde{\mu}(\mathrm{d} t, \mathrm{~d} e)=: \sum_{n \in \mathbb{N}} \int_{0}^{\cdot} \alpha_{t}^{n} \mathrm{~d} L_{t}^{n}=\sum_{n \in \mathbb{N}} \alpha^{n} \cdot L^{n},
$$

for $\alpha_{t}^{n}:=\left\langle U_{t}, u^{n}\right\rangle$ and $L^{n}:=u^{n} * \widetilde{\mu}$. Indeed, setting $F_{t}^{n}:=\sum_{k=1}^{n}\left\langle U_{t}, u^{k}\right\rangle u^{k}=\sum_{k=1}^{n} \alpha_{t}^{k} u^{k}$ one sees that $\left\|\sum_{k=1}^{\infty}\left|\alpha^{k}\right|^{2}\right\|_{L^{1}(\mathbb{P} \otimes \mathrm{d} t)} \leq\|U\|_{\mathcal{L}^{2}(\widetilde{\mu})}^{2}<\infty$. By dominated convergence one obtains as $n \rightarrow \infty$

$$
\left\|F^{n}-U\right\|_{\mathcal{L}^{2}(\tilde{\mu})}^{2}=\mathbb{E}\left(\int_{0}^{T} \int_{E}\left|F_{t}^{n}(e)-U_{t}(e)\right|^{2} \lambda(\mathrm{d} e) \mathrm{d} t\right)=\mathbb{E}\left(\int_{0}^{T} \sum_{k=n+1}^{\infty}\left|\alpha_{t}^{k}\right|^{2} \mathrm{~d} t\right) \rightarrow 0 .
$$

Isometry implies that the stochastic integrals $F^{n} * \widetilde{\mu}$ converge to $U * \widetilde{\mu}$ in $\mathcal{M}^{2}$, proving (2.3). In particular, we see how the PRP (2.2) w.r.t. a random measure can be rewritten as series of ordinary stochastic integrals w.r.t. scalar-valued strongly orthogonal martingales $L^{n}$, which are in fact Lévy processes with deterministic characteristics $\left(0,0, \int u^{n}(e) \lambda(\mathrm{d} e)\right)$. In this sense, the general condition (2.2) links well with results on PRP and BSDEs for Lévy processes in [NS00, NS01] who study a specific Teugels martingale basis consisting of compensated power jump processes for Lévy processes which satisfy exponential moment conditions. For a systematic analysis of related PRP results, comprising general Lévy processes, see [DTE15b, DTE15a].

6. Previous arguments could extend to the general case with $\zeta \neq \equiv 1$ in (2.1). To this end, suppose $U^{n}$ to be in $\mathcal{L}^{2}(\widetilde{\mu})$ such that for all $t \leq T$ the sequence $\left(U_{t}^{n}\right)_{n \in \mathbb{N}}$ is ONB of $L^{2}\left(\lambda_{t}\right)$ for $\mathrm{d} \lambda_{t}=\zeta_{t} \mathrm{~d} \lambda$ with scalar product $\langle u, v\rangle_{t}:=\int_{E} u(e) v(e) \zeta(t, e) \lambda(\mathrm{d} e)$. Analogously to case 5 . above, with $\alpha_{t}^{n}:=\left\langle U_{t}, U_{t}^{n}\right\rangle_{t}$ and $L^{n}:=U^{n} * \widetilde{\mu}$ one gets equalities of martingales (in $\mathcal{M}^{2}$ )

$$
U * \widetilde{\mu}=\sum_{n \in \mathbb{N}} \int_{0}\left\langle U_{t}, U_{t}^{n}\right\rangle_{t} \int_{E} U_{t}^{n}(e) \widetilde{\mu}(\mathrm{d} t, \mathrm{~d} e)=: \sum_{n \in \mathbb{N}} \alpha^{n} \cdot L^{n} .
$$

To proceed, we now define a solution of the Backward SDE with jumps to be a triple $(Y, Z, U)$ of processes in the space $\mathcal{S}^{p} \times \mathcal{L}^{2}(B) \times \mathcal{L}^{2}(\widetilde{\mu})$ for a suitable $p \in(1, \infty]$ that satisfies

$$
Y_{t}=\xi+\int_{t}^{T} f_{s}\left(Y_{s-}, Z_{s}, U_{s}\right) \mathrm{d} s-\int_{t}^{T} Z_{s} \mathrm{~d} B_{s}-\int_{t}^{T} \int_{E} U_{s}(e) \widetilde{\mu}(\mathrm{d} s, \mathrm{~d} e), \quad 0 \leq t \leq T,
$$

for given data $(\xi, f)$, consisting of a $\mathcal{F}_{T}$-measurable random variable $\xi$ and a generator function $f_{t}(y, z, u)=f(\omega, t, y, z, u)$. The values $p$ will be specified below in the respective results, although a particular focus will be on bounded BSDE solutions (i.e. $p=\infty$ ). Because we permit $\nu$ to be time-inhomogeneous with a bounded but possibly non-constant density $\zeta$ in (2.1), it does not hold in general that $U_{t}$ takes values a.e. in one space $L^{2}(\lambda)$ for $U \in \mathcal{L}^{2}(\widetilde{\mu})$. This requires some extra consideration about the domain of definition and measurability of $f$, as the generator function $f$ needs to be defined for $u$-arguments from a suitable domain, which cannot be some fixed $L^{2}$-space in general (and needs to be larger than $L^{2}(\lambda)$ ), as integrability of $u=U_{t}(\omega, \cdot)$ over $e \in E$ may vary with $(\omega, t)$. On suitable larger domains, one typically may have to admit for $f$ to attain non-finite values. To this end, let us denote by $L^{0}(\mathcal{B}(E), \lambda)$ the space of all $\mathcal{B}(E)$-measurable functions with the topology of convergence in measure and define

$$
\left|u-u^{\prime}\right|_{t}:=\left(\int_{E}\left|u(e)-u^{\prime}(e)\right|^{2} \zeta(t, e) \lambda(\mathrm{d} e)\right)^{\frac{1}{2}}
$$

for functions $u, u^{\prime}$ in $L^{0}(\mathcal{B}(E), \lambda)$. Terminal conditions $\xi$ for BSDE considered in this paper will be taken to be square integrable $\xi \in L^{2}\left(\mathcal{F}_{T}\right)$ and often even as bounded $\xi \in L^{\infty}\left(\mathcal{F}_{T}\right)$. Generator functions $f: \Omega \times[0, T] \times \mathbb{R} \times \mathbb{R}^{d} \times L^{0}(\mathcal{B}(E), \lambda) \rightarrow \overline{\mathbb{R}}$ are always taken to be $\mathcal{P} \otimes \mathcal{B}\left(\mathbb{R}^{d+1}\right) \otimes$ 
$\mathcal{B}\left(L^{0}(\mathcal{B}(E), \lambda)\right)$-measurable. Main Theorems 3.9 and 4.13 are derived for families of generators having the form

$$
f_{t}(y, z, u):=\widehat{f}_{t}(y, z)+\int_{A} g_{t}(y, z, u(e), e) \zeta(t, e) \lambda(\mathrm{d} e) \quad \text { (where finitely defined) }
$$

and $f_{t}(y, z, u):=\infty$ elsewhere, or more specially (for a $g$-component not depending on $y, z$ )

$$
f_{t}(y, z, u):=\widehat{f}_{t}(y, z)+\int_{A} g_{t}(u(e), e) \zeta(t, e) \lambda(\mathrm{d} e) \quad \text { (where finitely defined) }
$$

and $f_{t}(y, z, u):=\infty$ elsewhere, for a $\mathcal{B}(E)$-measurable set $A$ and component functions $\widehat{f}, g$ where $\widehat{f}: \Omega \times[0, T] \times \mathbb{R}^{1+d} \rightarrow \mathbb{R}$ is $\mathcal{P} \otimes \mathcal{B}\left(\mathbb{R}^{d+1}\right)$-measurable and $g: \Omega \times[0, T] \times \mathbb{R}^{1+d} \times \mathbb{R} \times E \rightarrow \mathbb{R}$ is $\mathcal{P} \otimes \mathcal{B}\left(\mathbb{R}^{d+2}\right) \otimes \mathcal{B}(E)$-measurable. Clearly statements for generators of the form (2.6) are also true for those of the (more particular) form (2.7). (In)finite activity relates to generators with $\lambda(A)<\infty$ (respectively $\lambda(A)=\infty$ ). A simple but useful technical Lemma clarifies how we can (and always will) choose a bounded representative for $U$ in a $\operatorname{BSDE}$ solution $(Y, Z, U)$ with bounded $Y$.

Lemma 2.2. Let $(Y, Z, U) \in \mathcal{S}^{\infty} \times \mathcal{L}^{2}(B) \times \mathcal{L}^{2}(\widetilde{\mu})$ be a solution of some JBSDE (2.4) with data $(\xi, f)$. Then there exists a representative $U^{\prime}$ of $U$, bounded pointwise by $2|Y|_{\infty}$, such that $U^{\prime}=U$ in $\mathcal{L}^{2}(\widetilde{\mu})$ and $\mathbb{P} \otimes \mathrm{d} t$-a-e., and $\left(Y, Z, U^{\prime}\right)$ solves the $B S D E(\xi, f)$.

Proof. We reproduce a brief argument sufficient to our general setting, similarly to e.g. [Mor09, Cor.1] or [Bec06, proof of Thm.3.5]. Use that $\mu(\omega, \mathrm{d} t, \mathrm{~d} e)=\sum_{s>0} \mathbb{1}_{D}(\omega, s) \delta_{\left(s, \beta_{s}(\omega)\right)}(\mathrm{d} t, \mathrm{~d} e)$ for an optional $E$-valued process $\beta$ and a thin set $D$, since $\mu$ is an integer-valued random measure [JS03, II.§1b]. Clearly the jump $\Delta Y_{t}(\omega)=\left(Y_{t}-Y_{t-}\right)(\omega)=\int_{E} U_{t}(\omega, e) \mu(\omega ;\{t\}$, de) is equal to $\mathbb{1}_{D}(\omega, t) U_{t}\left(\omega, \beta_{t}(\omega)\right)$ and bounded by $2|Y|_{\infty}$. For $U_{t}^{\prime}(\omega, e):=U_{t}(\omega, e) \mathbb{1}_{D}(\omega, t) \mathbb{1}_{\left\{\beta_{t}\right\}}(e)$, we have $U_{t}\left(\omega, \beta_{t}(\omega)\right)=U_{t}^{\prime}\left(\omega, \beta_{t}(\omega)\right)$ on $D$, and $\sum_{s \geq 0} \mathbb{1}_{D}(\omega, s)\left|U_{s}-U_{s}^{\prime}\right|^{2}\left(\omega, \beta_{s}(\omega)\right)=0$ implies $E\left[\left|U-U^{\prime}\right|^{2} *\right.$ $\left.\nu_{T}\right]=E\left[\left|U-U^{\prime}\right|^{2} * \mu_{T}\right]=0$. Since $U=U^{\prime}$ in $\mathcal{L}^{2}(\widetilde{\mu})$ and $U_{t}=U_{t}^{\prime}$ in $L^{0}(\mathcal{B}(E), \lambda)$, the BSDE is solved by $\left(Y, Z, U^{\prime}\right)$.

Under these conditions, we can and will take $U$ to be bounded by twice the norm of $Y$; Defining $|U|_{\infty}:=\operatorname{ess} \sup _{(\omega, t, e)}\left|U_{t}(e)\right|$ for $U \in \mathcal{L}^{2}(\widetilde{\mu})$ yields $|U|_{\infty} \leq 2|Y|_{\infty}$ for any bounded BSDE solution $(Y, Z, U)$. The next lemma notes that the stochastic integrals of bounded JBSDE solutions are BMOmartingales when some truncated generator function is bounded from above (below) by $+(-)\langle M\rangle$ for a BMO-martingale $M$; Moreover, their BMO-norms depend only on $|Y|_{\infty}$, the BMO-norm of $M$ and the horizon T. See [Ken15, Lem.1.3] for details of the proof, and note that BMO-properties of integrals of (bounded) BSDEs are of course a well-studied topic, cf. [MC14] and references therein.

Lemma 2.3. Let $(Y, Z, U) \in \mathcal{S}^{\infty} \times \mathcal{L}^{2}(B) \times \mathcal{L}^{2}(\widetilde{\mu})$ be a bounded solution to the BSDE $(\xi, f)$. Assume there is $M \in \mathrm{BMO}(\mathbb{P})$ such that $\int_{t}^{T} f_{s}\left(Y_{s-}, Z_{s}, U_{s}\right) \mathrm{d} s \leq\langle M\rangle_{T}-\langle M\rangle_{t}$ or $-\int_{t}^{T} f_{s}\left(Y_{s-}, Z_{s}, U_{s}\right) \mathrm{d} s$ $\leq\langle M\rangle_{T}-\langle M\rangle_{t}$. Then $\int Z \mathrm{~d} B$ and $U * \widetilde{\mu}$ are BMO-martingales and their BMO-norms (resp. $L^{2}-$ norms) are bounded by a constant depending on $|Y|_{\infty}$ and $\|M\|_{\mathrm{BMO}(\mathbb{P})}$ (resp. on $\left.|Y|_{\infty},\|M\|_{\mathcal{M}^{2}}\right)$.

\section{Comparison theorems and a-priori-estimates}

The stage for the main comparison Theorem 3.9 and the a-priori- $L^{\infty}$-estimate of Proposition 3.11 in this section is set by the next proposition. Its line of proof follows the seminal Theorem 2.5 by[Roy06], with slight generalizations that are needed in the sequel. Just some details for the change of measure argument are elaborated a bit differently, measurable dependencies of the random field $\gamma$ are specified in more detail, and less is assumed on the generators. Instead of imposing specific conditions on the generators which imply existence of solutions, we only insist that we have solutions and impose a generalized $\left(\mathbf{A}_{\boldsymbol{\gamma}}\right)$-condition as explained in Example 3.8.1.

Proposition 3.1. Let $\left(Y^{i}, Z^{i}, U^{i}\right) \in \mathcal{S}^{\infty} \times \mathcal{L}^{2}(B) \times \mathcal{L}^{2}(\widetilde{\mu})$ be solutions to the BSDE (2.4) for data $\left(\xi_{i}, f_{i}\right), i=1,2$. Assume that $f_{2}$ is Lipschitz continuous w.r.t. $y$ and $z$. Let $\gamma: \Omega \times[0, T] \times \mathbb{R}^{d+3} \times E \rightarrow$ 
$[-1, \infty)$ with $\left(\omega, t, y, z, u, u^{\prime}, e\right) \mapsto \gamma_{t}^{y, z, u, u^{\prime}}\left(\right.$ e) be a $\mathcal{P} \otimes \mathcal{B}\left(\mathbb{R}^{d+3}\right) \otimes \mathcal{B}(E)$-measurable function such that for $\bar{\gamma}:=\gamma_{-}^{Y_{-}^{2}, Z^{2}, U^{1}, U^{2}}$ it holds

$$
f_{2}\left(t, Y_{t-}^{2}, Z_{t}^{2}, U_{t}^{1}\right)-f_{2}\left(t, Y_{t-}^{2}, Z_{t}^{2}, U_{t}^{2}\right) \leq \int_{E} \bar{\gamma}_{t}(e)\left(U_{t}^{1}(e)-U_{t}^{2}(e)\right) \zeta(t, e) \lambda(\mathrm{d} e), \mathbb{P} \otimes \mathrm{d} t \text {-a.e. }
$$

and the stochastic exponential $\mathcal{E}\left(\int \beta \mathrm{d} B+\bar{\gamma} * \widetilde{\mu}\right)$ is a martingale for $\beta$ from (3.2).

Then a comparison result holds, that means that the inequalities $\xi_{1} \leq \xi_{2}$ and $f_{1}\left(t, Y_{t-}^{1}, Z_{t}^{1}, U_{t}^{1}\right) \leq$ $f_{2}\left(t, Y_{t-}^{1}, Z_{t}^{1}, U_{t}^{1}\right), \mathbb{P} \otimes \mathrm{d} t$-a.e., together imply $Y_{t}^{1} \leq Y_{t}^{2}$ for all $t \leq T$.

In results like the above, in [Roy06] and further enchancements [QS13, KP16, Yao17], the key assumption needed for comparison is the existence of an abstract random field $\gamma$ such that inequalities are satisfied between processes. In contrast, the subsequent results of this section offer sufficient criteria for comparison that can be verified more easily by checking concrete dependencies w.r.t. to basically Euclidean arguments for generator functions $f$ of the type (2.6). See also [GS17] for a simpler version in a setting with a jump measure of Lévy-type on $E=\mathbb{R}^{1} \backslash\{0\}$ and $\zeta \equiv 1$.

Proof. We define $\widehat{\xi}:=\xi_{1}-\xi_{2}, \widehat{Y}:=Y^{1}-Y^{2}, \widehat{Z}:=Z^{1}-Z^{2}$ and $\widehat{U}:=U^{1}-U^{2}$. The processes

$$
\begin{aligned}
\alpha_{s} & :=\mathbb{1}_{\left\{Y_{s-}^{1} \neq Y_{s-}^{2}\right\}} \frac{f_{2}\left(s, Y_{s-}^{1}, Z_{s}^{1}, U_{s}^{1}\right)-f_{2}\left(s, Y_{s-}^{2}, Z_{s}^{1}, U_{s}^{1}\right)}{\left(Y_{s-}^{1}-Y_{s-}^{2}\right)} \\
\beta_{s} & :=\mathbb{1}_{\left\{Z_{s}^{1} \neq Z_{s}^{2}\right\}} \frac{f_{2}\left(s, Y_{s-}^{2}, Z_{s}^{1}, U_{s}^{1}\right)-f_{2}\left(s, Y_{s-}^{2}, Z_{s}^{2}, U_{s}^{1}\right)}{\left\|Z_{s}^{1}-Z_{s}^{2}\right\|^{2}}\left(Z_{s}^{1}-Z_{s}^{2}\right)
\end{aligned}
$$

and $R_{t}:=\exp \left(\int_{0}^{t} \alpha_{s} \mathrm{~d} s\right)$ are bounded due to the Lipschitz assumption on $f_{2}$. As in [Roy06], applying Itô's formula to $R \widehat{Y}$ between $\tau \wedge t$ and $\tau \wedge T$ for some stopping times $\tau$ yields

$$
\begin{aligned}
(R \widehat{Y})_{\tau \wedge t}= & (R \widehat{Y})_{\tau \wedge T}+\int_{\tau \wedge t}^{\tau \wedge T} R_{s}\left(f_{1}\left(s, Y_{s-}^{1}, Z_{s}^{1}, U_{s}^{1}\right)-f_{2}\left(s, Y_{s-}^{2}, Z_{s}^{2}, U_{s}^{2}\right)\right) \mathrm{d} s \\
& -\int_{\tau \wedge t}^{\tau \wedge T} R_{s} \widehat{Z}_{s} \mathrm{~d} B_{s}-\int_{\tau \wedge t}^{\tau \wedge T} \int_{E} R_{s} \widehat{U}_{s}(e) \widetilde{\mu}(\mathrm{d} s, \mathrm{~d} e)-\int_{\tau \wedge t}^{\tau \wedge T} R_{s} \alpha_{s} \widehat{Y}_{s-} \mathrm{d} s .
\end{aligned}
$$

Set $M:=\int R \widehat{Z} \mathrm{~d} B+(R \widehat{U}) * \widetilde{\mu}$ and $N:=\int \beta \mathrm{d} B+\bar{\gamma} * \widetilde{\mu}$. Then $\mathrm{d} \mathbb{Q}:=\mathcal{E}(N)_{T} \mathrm{~d} \mathbb{P}$ defines an absolutely continuous probability by the martingale property of the stochastic exponential $\mathcal{E}(N) \geq 0$; cf. [HWY92, Lem.9.40]. By Girsanov $L:=M-\langle M, N\rangle$ is a local $\mathbb{Q}$-martingale, and the inequality

$$
\begin{aligned}
f_{1}\left(s, Y_{s-}^{1}, Z_{s}^{1}, U_{s}^{1}\right)- & f_{2}\left(s, Y_{s-}^{2}, Z_{s}^{2}, U_{s}^{2}\right) \leq \alpha_{s} \widehat{Y}_{s-}+\beta_{s} \widehat{Z}_{s}+\int_{E} \bar{\gamma}_{s}(e) \widehat{U}_{s}(e) \zeta_{s}(e) \lambda(\mathrm{d} e) \mathbb{P} \otimes \mathrm{d} s \text {-a.e. } \\
& \text { implies }(R \widehat{Y})_{\tau \wedge t} \leq(R \widehat{Y})_{\tau \wedge T}-\left(L_{T}^{\tau}-L_{t}^{\tau}\right)
\end{aligned}
$$

Localizing $L$ along a sequence of stopping times $\tau_{n} \uparrow \infty$ and taking conditional expectations, we obtain $\mathbb{E}_{\mathbb{Q}}\left((R \widehat{Y})_{t \wedge \tau^{n}} \mid \mathcal{F}_{t}\right) \leq \mathbb{E}_{\mathbb{Q}}\left((R \widehat{Y})_{\tau^{n} \wedge T} \mid \mathcal{F}_{t}\right)$ for each $n \in \mathbb{N}$. Dominated convergence yields the estimate $R_{t} \widehat{Y}_{t} \leq \mathbb{E}_{\mathbb{Q}}\left(R_{T} \widehat{\xi} \mid \mathcal{F}_{t}\right) \leq 0$ and thus $Y_{t}^{1} \leq Y_{t}^{2}$.

Remark 3.2. $\quad$ 1. Switching roles of $f_{1}$ and $f_{2}$, one gets that if $f_{1}$ is Lipschitz in $y, z$ and satisfies (3.1) instead of $f_{2}$, then $\xi_{1} \leq \xi_{2}$ and $f_{1}\left(t, Y_{t-}^{2}, Z_{t}^{2}, U_{t}^{2}\right) \leq f_{2}\left(t, Y_{t-}^{2}, Z_{t}^{2}, U_{t}^{2}\right)$ imply $Y_{t}^{1} \leq Y_{t}^{2}$.

2. The result of Proposition 3.1 remains valid (with a similar proof) if one requires that the $Y$-components of JBSDE solutions to compare are in $\mathcal{S}^{2}$ instead of $\mathcal{S}^{\infty}$, and the stochastic exponential $\mathcal{E}(\beta \bullet B+\bar{\gamma} * \widetilde{\mu})$ is in $\mathcal{S}^{2}$. However, as it is stated, Proposition 3.1 is exactly what we will need to apply in the sequel to derive, e.g., Proposition 4.3 and Theorem 4.13.

Example 3.3. Sufficient conditions for $\mathcal{E}(\bar{\gamma} * \widetilde{\mu})$ to be a martingale are, for instance,

1. $\Delta(\bar{\gamma} * \widetilde{\mu})>-1$ and $\mathbb{E}\left(\exp \left(\langle\bar{\gamma} * \widetilde{\mu}\rangle_{T}\right)\right)=\mathbb{E}\left(\exp \left(\int_{0}^{T} \int_{E}\left|\bar{\gamma}_{s}(e)\right|^{2} \nu(\mathrm{d} s, \mathrm{~d} e)\right)\right)<\infty ;$ see [PS08, Thm.9]. This holds i.p. if $\int_{E}\left|\bar{\gamma}_{s}(e)\right|^{2} \zeta(s, e) \lambda(\mathrm{d} e)<$ const. $<\infty \mathbb{P} \otimes \mathrm{d}$ s-a.e. and $\bar{\gamma}>-1$.

2. $\Delta(\bar{\gamma} * \widetilde{\mu}) \geq-1+\delta$ for $\delta>0$ and $\bar{\gamma} * \widetilde{\mu}$ is a $\mathrm{BMO}(\mathbb{P})$-martingale due to Kazamaki [Kaz79]. 
3. $\Delta(\bar{\gamma} * \widetilde{\mu}) \geq-1$ and $\bar{\gamma} * \widetilde{\mu}$ is a uniformly integrable martingale and $\mathbb{E}\left(\exp \left(\langle\bar{\gamma} * \widetilde{\mu}\rangle_{T}\right)\right)<\infty$; see [LM78, Thm.I.8]. Such a condition is satisfied when $\bar{\gamma}$ is bounded and $|\bar{\gamma}| \leq \psi, \mathbb{P} \otimes \mathrm{d} t \otimes \lambda$-a.e. for a function $\psi \in L^{2}(\lambda)$ and $\zeta \equiv 1$. The latter is what is required for instance in the comparison Thm.4.2 of [QS13].

Note that under above conditions, also the stochastic exponential $\mathcal{E}\left(\int \beta \mathrm{d} B+\bar{\gamma} * \widetilde{\mu}\right)$ for $\beta$ bounded and predictable is a martingale, as it is easily seen by Novikov's criterion.

Let us also refer to [CE15, Sections 19 and A.9] for related so-called balance conditions on generators for JBSDE comparison by change of measure arguments.

In the statement of Proposition 3.1, the dependence of the process $\bar{\gamma}$ on the BSDE solutions is not needed for the proof as the same result holds if $\bar{\gamma}$ is just a predictable process such that the estimate on the generator $f_{2}$ and the martingale property (3.1) hold. The further functional dependence is needed for the sequel, as required in the following

Definition 3.4. We say that an $\overline{\mathbb{R}}$-valued generator function $f$ satisfies condition $\left(\mathbf{A}_{\boldsymbol{\gamma}}\right)$ if there is a $\mathcal{P} \otimes \mathcal{B}\left(\mathbb{R}^{d+3}\right) \otimes \mathcal{B}(E)$-measurable function $\gamma: \Omega \times[0, T] \times \mathbb{R}^{d+3} \times E \rightarrow(-1, \infty)$ given by $\left(\omega, t, y, z, u, u^{\prime}, e\right) \mapsto \gamma_{t}^{y, z, u, u^{\prime}}(e)$ such that for all $\left(Y, Z, U, U^{\prime}\right) \in \mathcal{S}^{\infty} \times \mathcal{L}^{2}(B) \times\left(\mathcal{L}^{2}(\widetilde{\mu})\right)^{2}$ with $|U|_{\infty}<\infty,\left|U^{\prime}\right|_{\infty}<\infty$ it holds for $\bar{\gamma}:=\gamma^{Y_{-}, Z, U, U^{\prime}}$

$$
f_{t}\left(Y_{t-}, Z_{t}, U_{t}\right)-f_{t}\left(Y_{t-}, Z_{t}, U_{t}^{\prime}\right) \leq \int_{E} \bar{\gamma}_{t}(e)\left(U_{t}(e)-U_{t}^{\prime}(e)\right) \zeta(t, e) \lambda(\mathrm{d} e), \mathbb{P} \otimes \mathrm{d} t \text {-a.e. }
$$

and $\mathcal{E}\left(\int \beta \mathrm{d} B+\bar{\gamma} * \widetilde{\mu}\right)$ is a martingale for every bounded and predictable $\beta$.

We will say that $f$ satisfies condition $\left(\mathbf{A}_{\gamma}^{\prime}\right)$ if the above holds for all bounded $U$ and $U^{\prime}$ with additionally $U * \widetilde{\mu}$ and $U^{\prime} * \widetilde{\mu}$ in $\operatorname{BMO}(\mathbb{P})$.

Clearly, existence and applicability of a suitable comparison result for solutions to JBSDEs implies their uniqueness. In other words, if there exists a bounded solution for a generator being Lipschitz w.r.t. $y$ and $z$ which satisfies $\left(\mathbf{A}_{\boldsymbol{\gamma}}\right)$ or $\left(\mathbf{A}_{\gamma}^{\prime}\right)$, we obtain that such a solution is unique.

Example 3.5. The natural candidate for $\gamma$ for generators $f$ of the form (2.6) is given by

$$
\gamma_{s}^{y, z, u, u^{\prime}}(e)=\frac{g_{s}(y, z, u, e)-g_{s}\left(y, z, u^{\prime}, e\right)}{u-u^{\prime}} \mathbb{1}_{A}(e) \mathbb{1}_{\left\{u \neq u^{\prime}\right\}}
$$

which is $\mathcal{P} \otimes \mathcal{B}\left(\mathbb{R}^{d+3}\right) \otimes \mathcal{B}(E)$-measurable since $g$ is. Assuming absolute continuity of $g$ in $u$, we can express $\gamma_{s}^{y, z, u, u^{\prime}}(e)=\int_{0}^{1} \frac{\partial}{\partial u} g_{s}\left(y, z, t u+(1-t) u^{\prime}, e\right) \mathrm{d} t \mathbb{1}_{A}(e)$, by noting that

$$
\left(u-u^{\prime}\right) \int_{0}^{1} \frac{\partial}{\partial u} g_{s}\left(y, z, t u+(1-t) u^{\prime}, e\right) \mathrm{d} t \mathbb{1}_{A}(e)=\int_{0}^{1} \frac{\partial}{\partial t}\left[\left(g_{s}\left(y, z, t u+(1-t) u^{\prime}, e\right)\right)\right] \mathrm{d} t \mathbb{1}_{A}(e) .
$$

For generators of type (2.7) the $\gamma$ simply is $\gamma_{s}^{y, z, u, u^{\prime}}(e)=\int_{0}^{1} \frac{\partial}{\partial u} g_{s}\left(t u+(1-t) u^{\prime}, e\right) \mathrm{d} t \mathbb{1}_{A}(e)$.

Definition 3.6. We say that a generator $f$ satisfies condition $\left(\mathbf{A}_{\mathbf{f i n}}\right)$ or $\left(\mathbf{A}_{\mathbf{i n f}}\right)$ (on a set $\left.D\right)$ if

1. $\left(\mathbf{A}_{\mathbf{f i n}}\right): f$ is of the form (2.6) with $\lambda(A)<\infty$, is Lipschitz continuous w.r.t. $y$ and $z$ uniformly in $(t, \omega, u)$, and the map $u \mapsto g(t, y, z, u, e)$ is absolutely continuous (in $u$ ) for all $(\omega, t, y, z, e)$ (in $D \subseteq \Omega \times[0, T] \times \mathbb{R} \times \mathbb{R}^{d} \times E$ ), i.e. $g(t, y, z, u, e)=g(0)+\int_{0}^{u} g^{\prime}(t, y, z, x, e) \mathrm{d} x$, with density function $g^{\prime}$ being strictly greater than -1 (on D) and locally bounded (in u) from above, uniformly in $(\omega, t, y, z, e)$.

2. $\left(\mathbf{A}_{\mathbf{i n f i}}\right)$ : $f$ is of the form (2.7), is Lipschitz continuous w.r.t. $y$ and $z$ uniformly in $(t, \omega, u)$, and the map $u \mapsto g_{t}(u, e)$ is absolutely continuous (in $\left.u\right)$ for all $(\omega, t, e)$ (in D), i.e. $g(t, u, e)=$ $g(0)+\int_{0}^{u} g^{\prime}(t, x, e) \mathrm{d} x$, with density function $g^{\prime}$ being such that for all $c \in(0, \infty)$ there exists $K(c) \in \mathbb{R}$ and $\delta(c) \in(0,1)$ with $-1+\delta(c) \leq g^{\prime}(x)$ and $\left|g^{\prime}(x)\right| \leq K(c)|x|$ for all $x$ with $|x| \leq c$.

Remark 3.7. Note that under condition $\left(\mathbf{A}_{\mathbf{i n f}}\right)$ the density function $g^{\prime}$ is necessarily locally bounded, in particular with $\left|g^{\prime}(x)\right| \leq K(c) c=: \bar{K}(c)<\infty$ for all $x \in[-c, c]$. Observe that the conditions are not requiring the function $g$ to be convex and moreover refrain from requiring it to be continuously differentiable in $u$. Both can be helpful in application examplres, see Section 5.1.2. 
Example 3.8. Sufficient conditions for condition $\left(\mathbf{A}_{\boldsymbol{\gamma}}\right)$ and $\left(\mathbf{A}_{\boldsymbol{\gamma}}^{\prime}\right)$ are

1. $\gamma$ is a $\mathcal{P} \otimes \mathcal{B}\left(\mathbb{R}^{d+3}\right) \otimes \mathcal{B}(E)$-measurable function satisfying the inequality in (3.4) and

$$
C_{1}(1 \wedge|e|) \leq \gamma_{t}^{y, z, u, u^{\prime}}(e) \leq C_{2}(1 \wedge|e|)
$$

on $E=\mathbb{R}^{l} \backslash\{0\}(l \in \mathbb{N})$, for some $C_{1} \in(-1,0]$ and $C_{2}>0$. In this case $\exp \left(\left\langle\int \beta \mathrm{d} B+\bar{\gamma} * \widetilde{\mu}\right\rangle_{T}\right)$ $i$ clearly bounded and the jumps of $\int \beta \mathrm{d} B+\bar{\gamma} * \widetilde{\mu}$ are bigger than -1 . Hence $\mathcal{E}\left(\int \beta \mathrm{d} B+\bar{\gamma} * \widetilde{\mu}\right)$ is a positive martingale [PS08, Thm.9]. Thus Definition 3.4 generalizes the original $\left(\mathbf{A}_{\gamma}\right)$ condition introduced by [Roy06] for Poisson random measures.

2. $\left(\mathbf{A}_{\mathbf{f i n}}\right)$ is sufficient for $\left(\mathbf{A}_{\boldsymbol{\gamma}}\right)$. This follows from Example 3.3.1, (3.5) and $\lambda(A)<\infty$.

3. ( $\left.\mathbf{A}_{\mathbf{i n f i}}\right)$ is sufficient for $\left(\mathbf{A}_{\gamma}^{\prime}\right)$. To see this, let $u, u^{\prime}$ be bounded by $c$ and $\gamma$ be the natural candidate in Example 3.5. Then $\left|\gamma_{s}^{y, z, u, u^{\prime}}(e)\right| \leq \int_{u^{\prime}}^{u}\left|g^{\prime}(x)\right| \mathrm{d} x /\left(u-u^{\prime}\right) \leq K(c)\left(|u|+\left|u^{\prime}\right|\right)$. Hence $\int \beta \mathrm{d} B+\bar{\gamma} * \widetilde{\mu}$ is a BMO-martingale by the BMO-property of $U * \widetilde{\mu}$ and $U^{\prime} * \widetilde{\mu}$ with some lower bound $-1+\delta$ for its jumps. And $\mathcal{E}\left(\int \beta \mathrm{d} B+\bar{\gamma} * \widetilde{\mu}\right)$ is a martingale by part 2 of Example 3.3.

4. Condition ( $\left.\mathbf{A}_{\mathbf{f i n}}\right)$ above is satisfied if, e.g., $f$ is of the form (2.6) with $\lambda(A)<\infty$, is Lipschitz continuous w.r.t. $y$ and $z$, and the map $u \mapsto g(t, y, z, u, e)$ is continuously differentiable for all $(\omega, t, y, z, e)$ (in $D)$ such that the derivative is strictly greater than -1 (on $D \subseteq$ $\Omega \times[0, T] \times \mathbb{R} \times \mathbb{R}^{d} \times E$ ) and locally bounded (in u) from above, uniformly in $(\omega, t, y, z, e)$.

5. Condition ( $\left.\mathbf{A}_{\mathbf{i n f i}}\right)$ is valid if for instance $f$ is of the form (2.7), is Lipschitz continuous w.r.t. $y$ and $z$, and the map $u \mapsto g_{t}(u, e)$ is twice continuously differentiable for all $(\omega, t, e)$ with the derivatives being locally bounded uniformly in $(\omega, t, e)$, the first derivative being (locally) bounded away from -1 with a lower bound $-1+\delta$ for some $\delta>0$, and $\frac{\partial g}{\partial u}(t, 0, e) \equiv 0$.

As an application of the above, we can now provide simple conditions for comparison in terms of concrete properties of the generator function, which are easier to verify than the more general but abstract conditions on the existence of a suitable function $\gamma$ as in Proposition 3.1 or the general conditions by [CE10]. Note that no convexity is required in the $z$ or $u$ argument of the generator. The result will be applied later to prove existence and uniqueness of JBSDE solutions.

Theorem 3.9 (Comparison Theorem). A comparison result between bounded BSDE solutions in the sense of Proposition 3.1 holds true in each of the following cases:

1. (finite activity) $f_{2}$ satisfies $\left(\mathbf{A}_{\mathbf{f i n}}\right)$.

2. (infinite activity) $f_{2}$ satisfies $\left(\mathbf{A}_{\text {infi }}\right)$ and $U^{1} * \widetilde{\mu}$ and $U^{2} * \widetilde{\mu}$ are $\mathrm{BMO}(\mathbb{P})$-martingales for the corresponding JBSDE solutions $\left(Y^{1}, Z^{1}, U^{1}\right)$ and $\left(Y^{2}, Z^{2}, U^{2}\right)$.

Proof. This follows directly from Proposition 3.1 and Example 3.8, noting that representation (3.5) in connection with condition $\left(\mathbf{A}_{\mathbf{f i n}}\right)$ resp. $\left(\mathbf{A}_{\mathbf{i n f}}\right)$ meets the sufficient conditions in Example 3.3.

Unlike classical a-priori estimates that offer some $L^{2}$-norm estimates for the BSDE solution in terms of the data, the next result gives a simple $L^{\infty}$-estimate for the $Y$-component of the solution. Such will be useful for the derivation of BSDE solution bounds and for truncation arguments.

Proposition 3.10. Let $(Y, Z, U) \in \mathcal{S}^{\infty} \times \mathcal{L}^{2}(B) \times \mathcal{L}^{2}(\widetilde{\mu})$ be a solution to the BSDE $(\xi, f)$ with $\xi \in L^{\infty}\left(\mathcal{F}_{T}\right), f$ be Lipschitz continuous w.r.t. $(y, z)$ with Lipschitz constant $K_{f}^{y, z}$ and satisfying $\left(\mathbf{A}_{\gamma}\right)$ with $f .(0,0,0)$ bounded. Then $\left|Y_{t}\right| \leq \exp \left(K_{f}^{y, z}(T-t)\right)\left(|\xi|_{\infty}+(T-t)|f .(0,0,0)|_{\infty}\right)$ for $t \leq T$.

Proof. Set $\left(Y^{1}, Z^{1}, U^{1}\right)=(Y, Z, U),\left(\xi^{1}, f^{1}\right)=(\xi, f),\left(Y^{2}, Z^{2}, U^{2}\right)=(0,0,0)$ and $\left(\xi^{2}, f^{2}\right)=(0, f)$. Then following the proof of Proposition 3.1, equation (3.3) becomes

$$
(R Y)_{\tau \wedge t} \leq(R Y)_{\tau \wedge T}+\int_{\tau \wedge t}^{\tau \wedge T} R_{s} f_{s}(0,0,0) \mathrm{d} s-\left(L_{T}^{\tau}-L_{t}^{\tau}\right), \quad t \in[0, T]
$$


for all stopping times $\tau$ where $L:=M-\langle M, N\rangle$ is in $\mathcal{M}_{\text {loc }}(\mathbb{Q}), M:=\int R Z \mathrm{~d} B+(R U) * \widetilde{\mu}$ is in $\mathcal{M}^{2}, N:=\int \beta \mathrm{d} B+\bar{\gamma} * \widetilde{\mu}$ with $\bar{\gamma}:=\gamma^{0,0, U, 0}$ and the probability measure $\mathbb{Q} \approx \mathbb{P}$ is given by $\mathrm{d} \mathbb{Q}:=$ $\mathcal{E}(N)_{T} \mathrm{~d} \mathbb{P}$. Localizing $L$ along some sequence $\tau^{n} \uparrow \infty$ of stopping times yields $\mathbb{E}_{\mathbb{Q}}\left((R Y)_{\tau^{n} \wedge t} \mid \mathcal{F}_{t}\right) \leq$ $\mathbb{E}_{\mathbb{Q}}\left((R Y)_{\tau^{n} \wedge T}+\int_{\tau \wedge t}^{\tau \wedge T} R_{s} f_{s}(0,0,0) \mathrm{d} s \mid \mathcal{F}_{t}\right)$. By dominated convergence, we conclude that $\mathbb{P}$-a.e

$$
Y_{t} \leq \mathbb{E}_{\mathbb{Q}}\left(\frac{R_{T}}{R_{t}} \xi+\int_{t}^{T} \frac{R_{s}}{R_{t}} f_{s}(0,0,0) \mathrm{d} s \mid \mathcal{F}_{t}\right) \leq \mathrm{e}^{K_{f}^{y, z}(T-t)}\left(|\xi|_{\infty}+(T-t)|f .(0,0,0)|_{\infty}\right) .
$$

Analogously, if we define $\bar{N}:=\int \beta \mathrm{d} B+\overline{\widetilde{\gamma}} * \widetilde{\mu}$ with $\overline{\widetilde{\gamma}}:=\gamma^{0,0,0, U}$, and $\overline{\mathbb{Q}}$ equivalent to $\mathbb{P}$ via $\mathrm{d} \overline{\mathbb{Q}}:=\mathcal{E}(\bar{N})_{T} \mathrm{~d} \mathbb{P}$, we deduce that $\bar{L}:=M-\langle M, \bar{N}\rangle$ is in $\mathcal{M}_{\text {loc }}(\overline{\mathbb{Q}})$ and

$$
(R Y)_{\tau \wedge t} \geq(R Y)_{\tau \wedge T}+\int_{\tau \wedge t}^{\tau \wedge T} R_{s} f_{s}(0,0,0) \mathrm{d} s-\left(\bar{L}_{T}^{\tau}-\bar{L}_{t}^{\tau}\right), \quad t \in[0, T],
$$

for all stopping times $\tau$. This yields the required lower bound.

Again, we can specify explicit conditions on the generator function that are sufficient to ensure the more abstract assumptions of the previous result.

Proposition 3.11. Let $(Y, Z, U) \in \mathcal{S}^{\infty} \times \mathcal{L}^{2}(B) \times \mathcal{L}^{2}(\widetilde{\mu})$ be a solution to the BSDE $(\xi, f)$ with $\xi$ in $L^{\infty}\left(\mathcal{F}_{T}\right), f$ being Lipschitz continuous w.r.t. $(y, z)$ with Lipschitz constant $K_{f}^{y, z}$ such that $f .(0,0,0)$ is bounded. Assume that one of the following conditions holds:

1. (finite activity) $f$ satisfies $\left(\mathbf{A}_{\text {fin }}\right)$.

2. (infinite activity) $f$ satisfies $\left(\mathbf{A}_{\mathbf{i n f}}\right)$ and $U * \widetilde{\mu}$ is a $\mathrm{BMO}(\mathbb{P})$-martingale.

Then $\left|Y_{t}\right| \leq \exp \left(K_{f}^{y, z}(T-t)\right)\left(|\xi|_{\infty}+(T-t)\left|f_{s}(0,0,0)\right|_{\infty}\right)$ holds for all $t \leq T$, in particular $|Y|_{\infty} \leq \exp \left(K_{f}^{y, z} T\right)\left(|\xi|_{\infty}+T\left|f_{s}(0,0,0)\right|_{\infty}\right)$.

Proof. This follows directly from Proposition 3.10 and Example 3.8, since $f$ satisfies condition $\left(\mathbf{A}_{\boldsymbol{\gamma}}\right)$ (resp. $\left(\mathbf{A}_{\gamma}^{\prime}\right)$ ) using equation (3.5).

In the last part of this section we apply our comparison theorem for more concrete generators. To this end, we consider a generator $f$ being truncated at bounds $a<b$ (depending on time only) as

$$
\widetilde{f}_{t}(y, z, u):=f_{t}(\kappa(t, y), z, \kappa(t, y+u)-\kappa(t, y)),
$$

with $\kappa(t, y):=(a(t) \vee y) \wedge b(t)$. Next, we show that if a generator satisfies $\left(\mathbf{A}_{\gamma}\right)$ within the truncation bounds, then the truncated generator satisfies $\left(\mathbf{A}_{\gamma}\right)$ everywhere.

Lemma 3.12. Let $f$ satisfy (3.4) for $Y, U$ such that $a(t) \leq Y_{t-}, Y_{t-}+U_{t}(e), Y_{t-}+U_{t}^{\prime}(e) \leq b(t)$, $t \in[0, T]$ and let $\gamma$ satisfy one of the conditions of Example 3.3 for the martingale property of $\mathcal{E}(\bar{\gamma} * \widetilde{\mu})$. Then $\widetilde{f}$ satisfies (3.4). Especially, if $f$ satisfies $\left(\mathbf{A}_{\mathrm{fin}}\right)$ on the set where $a(t) \leq y, y+u \leq b(t)$ then $\widetilde{f}$ is Lipschitz in $(y, z)$, locally Lipschitz in $u$ and satisfies $\left(\mathbf{A}_{\gamma}\right)$.

Proof. Using monotonicity of $x \mapsto \kappa(t, x)$, we get that $\widetilde{f}_{t}\left(Y_{t-}, Z_{t}, U_{t}\right)-\widetilde{f}_{t}\left(Y_{t-}, Z_{t}, U_{t}^{\prime}\right)$ equals

$$
\begin{aligned}
& f_{t}\left(\kappa\left(t, Y_{t-}\right), Z_{t}, \kappa\left(t, Y_{t-}+U_{t}\right)-\kappa\left(t, Y_{t-}\right)\right)-f_{t}\left(\kappa\left(t, Y_{t-}\right), Z_{t}, \kappa\left(t, Y_{t-}+U_{t}^{\prime}\right)-\kappa\left(t, Y_{t-}\right)\right) \\
& \quad \leq \int_{E} \bar{\gamma}_{t}(e)\left(\kappa\left(t, Y_{t-}+U_{t}(e)\right)-\kappa\left(t, Y_{t-}+U_{t}^{\prime}(e)\right)\right) \zeta(t, e) \lambda(\mathrm{d} e) \\
& \quad \leq \int_{E} \bar{\gamma}_{t}(e)\left(\mathbb{1}_{\left\{\bar{\gamma} \geq 0, U \geq U^{\prime}\right\}}+\mathbb{1}_{\left\{\bar{\gamma}<0, U<U^{\prime}\right\}}\right)\left(U_{t}(e)-U_{t}^{\prime}(e)\right) \zeta(t, e) \lambda(\mathrm{d} e) .
\end{aligned}
$$

Setting $\overline{\gamma^{*}}:=\bar{\gamma}\left(\mathbb{1}_{\left\{\bar{\gamma} \geq 0, U \geq U^{\prime}\right\}}+\mathbb{1}_{\left\{\bar{\gamma}<0, U<U^{\prime}\right\}}\right)$ we see that the stochastic exponential $\mathcal{E}\left(\int \beta \mathrm{d} B+\overline{\gamma^{*}} * \widetilde{\mu}\right)$ is a martingale for all bounded and predictable processes $\beta$ and $\widetilde{f}$ satisfies (3.4). The latter claim easily follows from the fact that if $f$ satisfies $\left(\mathbf{A}_{\text {fin }}\right)$ on $a(t) \leq y, y+u \leq b(t)$ then $f$ satisfies (3.4) on $a(t) \leq Y_{t-}, Y_{t-}+U_{t}(e), Y_{t-}+U_{t}^{\prime}(e) \leq b(t)$ using Example 3.8.2. The Lipschitz properties of $\tilde{f}$ follow from the fact that $\kappa$ is a contraction and $f$ is Lipschitz within the truncation bounds. 
Concrete $L^{\infty}$-bounds for bounded solutions to $\operatorname{BSDE}(\xi, f)$ with suitable $\widehat{f}$-part are provided by

Proposition 3.13. Let $f$ be a generator of the form (2.6) with $\left|\widehat{f}_{t}(y, z)\right| \leq K_{1}+K_{2}|y|$ for some $K_{1}, K_{2} \geq 0, g_{t}(y, z, 0, e) \equiv 0$ and $\xi \in L^{\infty}\left(\mathcal{F}_{T}\right)$ with $c_{1} \leq \xi \leq c_{2}$ for some $c_{1}, c_{2} \in \mathbb{R}$. Assume that there are solutions $a$ and $b$ to the ODEs $y^{\prime}(t)=K_{1}+K_{2}|y(t)|, y(T)=c_{1}$ and $y^{\prime}(t)=-\left(K_{1}+K_{2}|y(t)|\right), y(T)=c_{2}$ respectively, such that $a \leq b$ on $[0, T]$. If the truncated generator $\widetilde{f}$ in (3.6) satisfies $\left(\mathbf{A}_{\boldsymbol{\gamma}}\right)$ and is Lipschitz in $(y, z)$, then any solution $(\widetilde{Y}, \widetilde{Z}, \widetilde{U}) \in \mathcal{S}^{\infty} \times \mathcal{L}^{2}(B) \times \mathcal{L}^{2}(\widetilde{\mu})$ to the $\operatorname{JBSDE}(\xi, \widetilde{f})$ also solves the $J B S D E(\xi, f)$ and satisfies $a(t) \leq \widetilde{Y}_{t} \leq b(t), t \in[0, T]$.

Proof. We set $Y_{t}:=\kappa\left(t, \widetilde{Y}_{t}\right), Z_{t}:=\widetilde{Z}_{t}, U_{t}(e):=\kappa\left(t, \widetilde{Y}_{t-}+\widetilde{U}_{t}(e)\right)-\kappa\left(t, \widetilde{Y}_{t-}\right)$ and

$$
f_{t}^{i}(y, z, u):=\widehat{f}_{t}^{i}(\kappa(t, y), z)+\int_{E} g_{t}(\kappa(t, y), z, \kappa(t, y+u)-\kappa(t, y), e) \zeta(t, e) \lambda(\mathrm{d} e)
$$

with $\widehat{f}_{t}^{1}(y, z):=-\left(K_{1}+K_{2}|y|\right), \widehat{f}_{t}^{2}(y, z):=\widehat{f}_{t}(y, z)$ and $\widehat{f}_{t}^{3}(y, z):=K_{1}+K_{2}|y|$. By the assumptions on the ODEs, we have that $(a(t), 0,0)$ solves the BSDE $\left(c_{1}, f^{1}\right)$ and $(b(t), 0,0)$ solves the BSDE $\left(c_{2}, f^{3}\right)$. Taking into account that $\widetilde{f}^{1} \leq \tilde{f}^{2} \leq \tilde{f}^{3}, c_{1} \leq \xi \leq c_{2}$ and $\tilde{f}^{2}$ satisfies $\left(\mathbf{A}_{\gamma}\right)$, comparison theorem Proposition 3.1 yields $a(t) \leq \widetilde{\widetilde{Y}}_{t} \leq b(t)$. Hence, $Y$ and $\widetilde{Y}$ are indistinguishable, $U=\widetilde{U}$ in $\mathcal{L}^{2}(\widetilde{\mu})$ and $(\widetilde{Y}, \widetilde{Z}, \widetilde{U})$ solves the $\operatorname{BSDE}(\xi, f)$.

In the next section, we apply these results to two situations: Using Corollary 4.4, we give an alternative proof of Thm.3.5 of [Bec06] via a comparison principle instead of an argument with stopping times. Moreover, the estimates in Corollary 4.6 are applied to solve the power utility maximization problem via a JBSDE approach in Section 5.2.

\section{Existence and uniqueness of bounded solutions}

This section studies BSDE with jumps by the monotone stability approach. Building on (straighforward) results for finite activity, the infinite activity case is treated by monotone approximations.

\subsection{The case of finite activity}

Definition 4.1. A generator function $f$ satisfies condition $\left(\mathbf{B}_{\boldsymbol{\gamma}}\right)$, if it is Lipschitz continuous in $(y, z)$, locally Lipschitz continuous in $u$ (in the sense that $u \mapsto f_{t}(y, z,-c \vee u \wedge c)$ is Lipschitz continuous for any $c \in(0, \infty)), f .(0,0,0)$ is bounded, and $f$ satisfies condition $\left(\mathbf{A}_{\boldsymbol{\gamma}}\right)$.

The next result readily leads to Proposition 4.3, for $A$ in $(2.6)$ with $\lambda(A)<\infty$.

Proposition 4.2. Let $\xi \in L^{\infty}\left(\mathcal{F}_{T}\right)$ and $f$ satisfies $\left(\mathbf{B}_{\boldsymbol{\gamma}}\right)$. Then there exists a unique solution $(Y, Z, U)$ in $\mathcal{S}^{\infty} \times \mathcal{L}^{2}(B) \times \mathcal{L}^{2}(\widetilde{\mu})$ to the BSDE $(\xi, f)$. Moreover for all $t \in[0, T],\left|Y_{t}\right|$ is bounded by $\exp \left(K_{f}^{y, z}(T-t)\right)\left(|\xi|_{\infty}+(T-t)|f .(0,0,0)|_{\infty}\right)$.

Proof. Consider the Lipschitz generator $f_{t}^{c}(y, z, u):=f_{t}(y, z,(u \vee(-c)) \wedge c)$ with $c>0$ and Lipschitz constant $K_{f^{c}}$. By classical fixed point arguments and a-priori estimates (cf. e.g. [Bec06, Prop.3.2, $3.3])$ there is a unique solution $\left(Y^{c}, Z^{c}, U^{c}\right) \in \mathcal{S}^{2} \times \mathcal{L}^{2}(B) \times \mathcal{L}^{2}(\widetilde{\mu})$ to the $\operatorname{BSDE}\left(\xi, f^{c}\right)$; it satisfies

$$
\left|Y_{t}^{c}\right| \leq C \mathbb{E}\left(|\xi|^{2}+\int_{t}^{T}\left|f_{s}^{c}(0,0,0)\right|^{2} \mathrm{~d} s \mid \mathcal{F}_{t}\right) \leq C\left(|\xi|_{\infty}^{2}+T|f .(0,0,0)|_{\infty}^{2}\right)<\infty
$$

for some constant $C=C\left(T, K_{f^{c}}\right)$. Now Proposition 3.10 implies that $\left|Y_{t}^{c}\right|$ is dominated by $\exp \left(K_{f}^{y, z}(T-t)\right)\left(|\xi|_{\infty}+(T-t)|f .(0,0,0)|_{\infty}\right)$ for all $c>0$. Choosing $c \geq 2 \exp \left(K_{f}^{y, z} T\right)\left(|\xi|_{\infty}+\right.$ $\left.T|f .(0,0,0)|_{\infty}\right)$ we get that $\left(Y^{c}, Z^{c}, U^{c}\right)$ with $Y^{c} \in \mathcal{S}^{\infty}$ solves the BSDE $(\xi, f)$ since $U^{c}$ is bounded by $c$. Uniqueness follows by comparison.

This leads to a preliminary result on bounded solutions if jumps are of finite activity.

Proposition 4.3. Let $\xi \in L^{\infty}\left(\mathcal{F}_{T}\right)$ and let $f$ satisfy $\left(\mathbf{A}_{\text {fin }}\right)$ (recall Definition 3.6) with $f .(0,0,0)$ bounded. Then there exists a unique solution $(Y, Z, U)$ in $\mathcal{S}^{\infty} \times \mathcal{L}^{2}(B) \times \mathcal{L}^{2}(\widetilde{\mu})$ to the $B S D E(\xi, f)$. Moreover for all $t \in[0, T],\left|Y_{t}\right|$ is bounded by $\exp \left(K_{f}^{y, z}(T-t)\right)\left(|\xi|_{\infty}+(T-t)|f .(0,0,0)|_{\infty}\right)$. 
Proof. Noting that local Lipschitz continuity in $u$ follows from the absolute continuity of $g$ in $u$ with locally bounded density function, the claim follows from Propositions 3.11 and 4.2.

Corollary 4.4. Let $\xi \in L^{\infty}\left(\mathcal{F}_{T}\right)$ and let $f$ be a generator satisfying $\left(\mathbf{A}_{\mathbf{f i n}}\right)$, with $g_{t}(y, z, 0, e) \equiv 0$ and $\left|\widehat{f}_{t}(y, z)\right| \leq K_{1}+K_{2}|y|$ for some $K_{1}, K_{2} \geq 0$. Set

$$
b(t)= \begin{cases}\left(|\xi|_{\infty}+\frac{K_{1}}{K_{2}}\right) \exp \left(K_{2}(T-t)\right)-\frac{K_{1}}{K_{2}}, & K_{2} \neq 0 \\ |\xi|_{\infty}+K_{1}(T-t), & K_{2}=0\end{cases}
$$

Then there exists a unique solution $(Y, Z, U) \in \mathcal{S}^{\infty} \times \mathcal{L}^{2}(B) \times \mathcal{L}^{2}(\widetilde{\mu})$ to the BSDE $(\xi, f)$ and moreover $\left|Y_{t}\right| \leq b_{t}$ for $t \in[0, T]$. Finally $\int Z \mathrm{~d} B$ and $U * \widetilde{\mu}$ are $\mathrm{BMO}(\mathbb{P})$-martingales.

Proof. By Lemma 3.12 and Proposition 4.3, there is a unique solution $(Y, Z, U)$ in the space $\mathcal{S}^{\infty} \times \mathcal{L}^{2}(B) \times \mathcal{L}^{2}(\widetilde{\mu})$ to the $\operatorname{BSDE}(\xi, \widetilde{f})$. By Proposition 3.13, it also solves the $\operatorname{BSDE}(\xi, f)$ and $-b(t) \leq Y_{t} \leq b(t), \forall t \in[0, T]$. Uniqueness follows from the fact that one can apply the comparison Theorem 3.9 for generators satisfying $\left(\mathbf{A}_{\mathbf{f i n}}\right)$. The BMO property follows from Lemma 2.3.

Remark 4.5. Corollary 4.4 is similar to Thm.3.5 in [Bec06], but its proof is different: It relies on previous comparison results for JBSDEs instead of stopping arguments. The stochastic integrals of the BSDE solution are BMO-martingales under the assumptions for Lemma 2.3, which hold e.g. under the conditions for [Bec06, Thm. 3.6]

Corollary 4.6. Let $\xi \in L^{\infty}\left(\mathcal{F}_{T}\right)$ with $\xi \geq C$ for some constant $C>0, K \geq 0$ and set $a(t):=$ $C \exp (-K(T-t))$ and $b(t)=|\xi|_{\infty} \exp (K(T-t)), \forall t \in[0, T]$. Assume $f$ satisfies $\left(\mathbf{A}_{\text {fin }}\right)$ for $c \leq y, y+u \leq d$ for all $c, d \in \mathbb{R}$ with $0<c<d$, and that $\left|\widehat{f}_{t}(y, z)\right| \leq K|y|$ and $g_{t}(y, z, 0, e)=0$. Then there exists a unique solution $(Y, Z, U) \in \mathcal{S}^{\infty} \times \mathcal{L}^{2}(B) \times \mathcal{L}^{2}(\widetilde{\mu})$ to the BSDE $(\xi, f)$ with $Y \geq \epsilon$ for some $\epsilon>0$. Moreover, it holds $a(t) \leq Y_{t} \leq b(t)$ and $\int Z \mathrm{~d} B$ and $U * \widetilde{\mu}$ are $\mathrm{BMO}(\mathbb{P})$-martingales.

Proof. This can be shown with a similar argument for the uniqueness as above: Let $\left(Y^{\prime}, Z^{\prime}, U^{\prime}\right)$ be another solution to the $\operatorname{BSDE}(\xi, f)$ with $Y^{\prime} \geq \epsilon$ for some $\epsilon>0$. Then $f$ satisfies $\left(\mathbf{A}_{\text {fin }}\right)$ for $a(t) \wedge \epsilon \leq y, y+u \leq b(t) \vee\left|Y^{\prime}\right|_{\infty}$; hence the solutions coincide by comparison.

Example 4.7. As a special case of Corollary 4.6 to be applied in Section 5.2, setting $K:=$ $\left(\gamma|\varphi|_{\infty}^{2}\right) /\left(2(1-\gamma)^{2}\right)$ for some $\gamma \in(0,1)$ and some predictable and bounded process $\varphi$ we define

$$
\begin{aligned}
f_{t}(y, z, u) & :=\widehat{f}_{t}(y, z)+\int_{E} g_{t}(y, u, e) \zeta(t, e) \lambda(\mathrm{d} e) \\
& :=\frac{\gamma}{2(1-\gamma)^{2}}\left|\varphi_{t}\right|^{2} y+\int_{E}\left(\frac{1}{1-\gamma}\left((u(e)+y)^{1-\gamma} y^{\gamma}-y\right)-u(e)\right) \zeta(t, e) \lambda(\mathrm{d} e) .
\end{aligned}
$$

From $\frac{\partial g}{\partial y}(t, y, u, e)=\left(\frac{u+y}{y}\right)^{1-\gamma}+\frac{\gamma}{1-\gamma}\left(\frac{u+y}{y}\right)^{-\gamma}-\frac{1}{1-\gamma}$, we see that $f$ is Lipschitz in $y$ within the truncation bounds. Moreover, $g$ is continuously differentiable with bounded derivatives and we have $\frac{\partial g}{\partial u}(t, y, u, e)=\left(\frac{u+y}{y}\right)^{-\gamma}-1>-1$, for $c \leq y, y+u \leq d$.

\subsection{The case of infinite activity}

For linear generators of the form $f_{t}(y, z, u):=\alpha_{t}^{0}+\alpha_{t} y+\beta_{t} z+\int_{E} \gamma_{t}(e) u(e) \zeta(t, e) \lambda(\mathrm{d} e)$, with predictable coefficients $\alpha^{0}, \alpha, \beta$ and $\gamma$, JBSDE solutions can be represented by an adjoint process. In our context of bounded solutions, one needs rather weak conditions on the adjoint process. This will be used later on in Section 5. The idea of proof is standard, cf. [Ken15, Lem.1.23] for details.

Lemma 4.8. Let $f$ be a linear generator of the form above and let $\xi$ be in $L^{\infty}\left(\mathcal{F}_{T}\right)$.

1. Assume that $(Y, Z, U) \in \mathcal{S}^{\infty} \times \mathcal{L}^{2}(B) \times \mathcal{L}^{2}(\widetilde{\mu})$ solves the BSDE $(\xi, f)$. Suppose that the adjoint process $\left(\Gamma_{s}^{t}\right)_{s \in[t, T]}:=\left(\exp \left(\int_{t}^{s} \alpha_{u} \mathrm{~d} u\right) \mathcal{E}\left(\int \beta \mathrm{d} B+\gamma * \widetilde{\mu}\right)_{t}^{s}\right)_{s \in[t, T]}$ is in $\mathcal{S}^{1}$ for any $t \leq T$ and $\alpha^{0}$ is bounded. Then $Y$ is represented as $Y_{t}=\mathbb{E}\left[\Gamma_{T}^{t} \xi+\int_{t}^{T} \Gamma_{s}^{t} \alpha_{s}^{0} \mathrm{~d} s \mid \mathcal{F}_{t}\right]$. 
2. Let $\alpha^{0}, \alpha, \beta$ and $\widetilde{\gamma}_{t}:=\int_{E}\left|\gamma_{t}(e)\right|^{2} \zeta(t, e) \lambda(\mathrm{d} e), t \in[0, T]$, be bounded and $\gamma \geq-1$. Then there is a unique solution in $\mathcal{S}^{\infty} \times \mathcal{L}^{2}(B) \times \mathcal{L}^{2}(\widetilde{\mu})$ to the $\operatorname{BSDE}(\xi, f)$ and Part 1. applies.

Our aim is to prove existence and uniqueness beyond Proposition 4.3 for infinite activity of jumps, that means $\lambda(A)$ may be infinite in (2.6). To show Theorems 4.11 and 4.13 , we use a monotone stability approach of [Kob00]: By approximating a generator $f$ of the form (2.7) (with $A$ such that $\lambda(A)=\infty$ ) by a sequence $\left(f^{n}\right)_{n \in \mathbb{N}}$ of the form (2.7) (with $A_{n}$ such that $\lambda\left(A_{n}\right)<\infty$ ) for which solutions' existence is guaranteed, one gets that the limit of these solutions exist and it solves the BSDE with the original data. As in [Kob00], the monotone approximation approach is perceived as being not easy in execution, a main problem usually being to prove strong convergence of the stochastic integral parts for the BSDE. By Proposition 4.9 convergence works for small terminal condition $\xi$. That is why we can not apply this Proposition directly to data $\left(\xi, f^{n}\right)_{n \in \mathbb{N}}$. Instead we sum (converging) solutions for small $1 / N$-fractions of the desired terminal condition. This is inspired by the iterative ansatz from [Mor10] for a particular generator. For our generator family, we adapt and elaborate proofs, using e.g. a $\mathcal{S}^{1}$-closeness argument for the proof of the strong approximation step. Compared to [Mor10], the analysis for our general family of JBSDEs adds clarity and structural insight into what is really needed. It extends the scope of the BSDE stability approach [Kob00, Mor10], in particular with regards to non-Lipschitz dependencies in the jump-integrand, while the proof shows comparable ease for the (usually laborious) strong approximation step in the setup under consideration. Differently to e.g. [EMN16, Mor10, Yao17], no exponential transforms or convolutions are needed here, as our generators are "quadratic" in $U$ but not in $Z$. Despite similarities at first sight, a closer look reveals that Theorem 4.11 is different from [KTPZ15, Thm.5.4], both in the method of proof and in scope: They prove existence for small terminal conditions by following the fixed point approach by [Tev08], whereas we show stability for small terminal conditions (Proposition 4.9) and apply a different pasting procedure, approximating not only terminal data but also generators. Here wellposedness of the approximating JBSDEs is obtained directly from classical theory by using comparison and estimates from Section 3, which enable us to argue within uniform a-priori bounds for the approximating sequence. Examples in Section 5 demonstrate that also the scope of our results is different.

In more detail, the task for the next Theorem 4.11 is to construct generators $\left(f^{k, n}\right)_{1 \leq k \leq N, n \in \mathbb{N}}$ and solutions $\left(Y^{k, n}, Z^{k, n}, U^{k, n}\right)$ to the BSDEs with data $\left(\xi / N, f^{k, n}\right)$ for $N$ large enough such that $\left(Y^{k, n}, Z^{k, n}, U^{k, n}\right)$ converges if $n \rightarrow \infty$ and $\left(Y^{n}, Z^{n}, U^{n}\right):=\sum_{k=1}^{N}\left(Y^{k, n}, Z^{k, n}, U^{k, n}\right)$ solves the $\operatorname{BSDE}\left(\xi, f^{n}\right)$. In this case $\left(Y^{n}, Z^{n}, U^{n}\right)$ converges and its limit is a solution candidate for the $\operatorname{BSDE}(\xi, f)$. For this program, we next show a stability result for JBSDE.

Proposition 4.9. Let $\left(\xi^{n}\right) \subset L^{\infty}\left(\mathcal{F}_{T}\right)$ with $\xi^{n} \rightarrow \xi$ in $L^{2}\left(\mathcal{F}_{T}\right)$ and $\left(f^{n}\right)_{n \in \mathbb{N}}$ be a sequence of generators with $f^{n}(0,0,0)=0, \forall n$, having property $\left(B_{\gamma^{n}}\right)$ such that $K_{f}^{y, z}:=\sup _{n \in \mathbb{N}} K_{f^{n}}^{y, z}<\infty$. Denote by $\left(Y^{n}, Z^{n}, U^{n}\right) \in \mathcal{S}^{\infty} \times \mathcal{L}^{2}(B) \times \mathcal{L}^{2}(\widetilde{\mu})$ the solution to the $B S D E\left(\xi, f^{n}\right)$ with $Y^{n}$ bounded by $|\xi|_{\infty} \exp \left(K_{f^{n}}^{y, z} T\right)$ and set $\tilde{c}:=|\xi|_{\infty} \exp \left(K_{f}^{y, z} T\right)$. Assume that $Y^{n}$ converges pointwise, $\left(Z^{n}, U^{n}\right) \rightarrow$ $(Z, U)$ converges weakly in $\mathcal{L}^{2}(B) \times \mathcal{L}^{2}(\widetilde{\mu})$ and $\left|f_{t}^{n}(0,0, u)\right| \leq \widehat{K}|u|_{t}^{2}+\widehat{L}_{t}$ for all $n$ and $u$ with $|u| \leq 2 \tilde{c}$, $\widehat{K} \in \mathbb{R}_{+}$and $\widehat{L} \in L^{1}(\mathbb{P} \otimes \mathrm{d} t)$. Then $\left(Z^{n}, U^{n}\right)$ converges to $(Z, U)$ strongly in $\mathcal{L}^{2}(B) \times \mathcal{L}^{2}(\widetilde{\mu})$, if $|\xi|_{\infty} \equiv \tilde{c} \exp \left(-K_{f}^{y, z} T\right) \leq \exp \left(-K_{f}^{y, z} T\right) /\left(80 \max \left\{K_{f}^{y, z}, \widehat{K}\right\}\right)$.

Proof. We note that $\left(Y^{n}, Z^{n}, U^{n}\right)$ is uniquely defined by Proposition 4.2. To prove strong convergence of $\left(Z^{n}\right)_{n \in \mathbb{N}}$ and $\left(U^{n}\right)_{n \in \mathbb{N}}$ we consider $\delta Y:=Y^{n}-Y^{m}, \delta Z:=Z^{n}-Z^{m}, \delta U:=U^{n}-U^{m}$ and apply Itô's formula for general semimartingales to $(\delta Y)^{2}$ to obtain

$$
\begin{aligned}
\left(\delta Y_{0}\right)^{2}= & \left(\delta Y_{T}\right)^{2}+\int_{0}^{T} 2 \delta Y_{s-}\left(f_{s}^{n}\left(Y_{s-}^{n}, Z_{s}^{n}, U_{s}^{n}\right)-f_{s}^{m}\left(Y_{s-}^{m}, Z_{s}^{m}, U_{s}^{m}\right)\right) \mathrm{d} s \\
& -\int_{0}^{T}\left\|\delta Z_{s}\right\|^{2} \mathrm{~d} s-2 \int_{0}^{T} \delta Y_{s-} \delta Z_{s} \mathrm{~d} B_{s}-\int_{0}^{T} \int_{E}\left(\delta Y_{s-}+\delta U_{s}(e)\right)^{2}-\left(\delta Y_{s-}\right)^{2} \widetilde{\mu}(\mathrm{d} s, \mathrm{~d} e) \\
& -\int_{0}^{T} \int_{E}\left(\delta Y_{s-}+\delta U_{s}(e)\right)^{2}-\left(\delta Y_{s-}\right)^{2}-2 \delta Y_{s-} \delta U_{s}(e) \nu(\mathrm{d} s, \mathrm{~d} e) .
\end{aligned}
$$


Noting that the stochastic integrals are martingales one concludes that

$$
\begin{aligned}
& \mathbb{E}\left(\int_{0}^{T} 2 \delta Y_{s-}\left(f_{s}^{n}\left(Y_{s-}^{n}, Z_{s}^{n}, U_{s}^{n}\right)-f_{s}^{m}\left(Y_{s-}^{m}, Z_{s}^{m}, U_{s}^{m}\right)\right) \mathrm{d} s\right) \\
& =\mathbb{E}\left(\int_{0}^{T} \int_{E} \delta U_{s}(e)^{2} \nu(\mathrm{d} s, \mathrm{~d} e)\right)+\mathbb{E}\left(\int_{0}^{T}\left\|\delta Z_{s}\right\|^{2} \mathrm{~d} s\right)-\mathbb{E}\left(\left(\delta Y_{T}\right)^{2}\right)+\mathbb{E}\left(\left(\delta Y_{0}\right)^{2}\right) .
\end{aligned}
$$

Using the inequalities $a \leq a^{2}+1 / 4,(a+b)^{2} \leq 2\left(a^{2}+b^{2}\right),(a+b+c)^{2} \leq 3\left(a^{2}+b^{2}+c^{2}\right)$, the Lipschitz property of $f^{n}$ in $y$ and $z$ and the estimate for $f_{t}^{n}(0,0, u)$, we have

$$
\begin{aligned}
& \left|f_{s}^{n}\left(Y_{s-}^{n}, Z_{s}^{n}, U_{s}^{n}\right)-f_{s}^{m}\left(Y_{s-}^{m}, Z_{s}^{m}, U_{s}^{m}\right)\right| \\
& \quad \leq K_{f^{n}}^{y, z}\left(\left|Y_{s-}^{n}\right|+\left\|Z_{s}^{n}\right\|\right)+K_{f^{m}}^{y, z}\left(\left|Y_{s-}^{m}\right|+\left\|Z_{s}^{m}\right\|\right)+\widehat{K}\left|U_{s}^{n}\right|_{s}^{2}+\widehat{L}_{s}+\widehat{K}\left|U_{s}^{m}\right|_{s}^{2}+\widehat{L}_{s} \\
& \quad \leq K_{1}+2 \widehat{L}_{s}+K_{2}\left(\left\|\delta Z_{s}\right\|^{2}+\left\|Z_{s}^{n}-Z_{s}\right\|^{2}+\left\|Z_{s}\right\|^{2}+\left|\delta U_{s}\right|_{s}^{2}+\left|U_{s}^{n}-U_{s}\right|_{s}^{2}+\left|U_{s}\right|_{s}^{2}\right),
\end{aligned}
$$

where $K_{1}:=K_{f}^{y, z}(2 \tilde{c}+1 / 2) \in \mathbb{R}, K_{2}:=5 \max \left\{K_{f}^{y, z}, \widehat{K}\right\}$ and $|\cdot|_{t}$ is defined in (2.5). Combing inequalities (4.1) and (4.2) yields

$$
\begin{array}{r}
\mathbb{E}\left(\int_{0}^{T}\left\|\delta Z_{s}\right\|^{2}+\left|\delta U_{s}\right|_{s}^{2} \mathrm{~d} s\right) \leq 2 \mathbb{E}\left(\int _ { 0 } ^ { T } | \delta Y _ { s - } | \left(K_{1}+2 \widehat{L}_{s}+K_{2}\left(\left\|\delta Z_{s}\right\|^{2}+\left\|Z_{s}^{n}-Z_{s}\right\|^{2}+\left\|Z_{s}\right\|^{2}\right.\right.\right. \\
\left.\left.\left.+\left|\delta U_{s}\right|_{s}^{2}+\left|U_{s}^{n}-U_{s}\right|_{s}^{2}+\left|U_{s}\right|_{s}^{2}\right)\right) \mathrm{~d} s\right)+\mathbb{E}\left(\left(\xi^{n}-\xi^{m}\right)^{2}\right) .
\end{array}
$$

Let us recall that the predictable projection of $Y$, denoted by $Y^{\mathrm{p}}$, is defined as the unique predictable process $X$ such that $X_{\tau}=\mathbb{E}\left(Y_{\tau} \mid \mathcal{F}_{\tau-}\right)$ on $\{\tau<\infty\}$ for all predictable times $\tau$. For $Y^{n}$ it holds $\left(Y^{n}\right)^{\mathrm{p}}=Y_{-}^{n}$. This follows from [JS03, Prop.I.2.35.] using that $Y^{n}$ is càdlàg, adapted and quasileft-continuous, as $\Delta Y_{\tau}=\Delta U * \widetilde{\mu}_{\tau}=0$ on $\{\tau<\infty\}$ holds for all predictable times $\tau$ thanks to the absolute continuity of the compensator $\nu$. Noting that $1-2 K_{2}\left|\delta Y_{s-}\right| \geq 1-4 K_{2} \tilde{c} \geq 3 / 4$ and setting $Y:=\lim _{n \rightarrow \infty} Y^{n}$ we deduce by the weak convergence of $\left(Z^{n}\right)_{n \in \mathbb{N}}$ and $\left(U^{n}\right)_{n \in \mathbb{N}}, Y_{-}^{n}=\left(Y^{n}\right)^{\mathrm{p} \uparrow}(Y)^{\mathrm{p}}$ as $n \rightarrow \infty$ and by Lebesgue's dominated convergence theorem

$$
\begin{aligned}
& \frac{3}{4} \mathbb{E}\left(\int_{0}^{T}\left\|Z_{s}^{n}-Z_{s}\right\|^{2}+\left|U_{s}^{n}-U_{s}\right|_{s}^{2} \mathrm{~d} s\right) \\
& \leq \frac{3}{4} \liminf _{m \rightarrow \infty} \mathbb{E}\left(\int_{0}^{T}\left\|Z_{s}^{n}-Z_{s}^{m}\right\|^{2}+\left|U_{s}^{n}-U_{s}^{m}\right|_{s}^{2} \mathrm{~d} s\right) \\
& \leq \liminf _{m \rightarrow \infty} 2 \mathbb{E}\left(\int_{0}^{T}\left|\delta Y_{s-}\right|\left(K_{1}+2 \widehat{L}_{s}+K_{2}\left(\left\|Z_{s}^{n}-Z_{s}\right\|^{2}+\left\|Z_{s}\right\|^{2}+\left|U_{s}^{n}-U_{s}\right|_{s}^{2}+\left|U_{s}\right|_{s}^{2}\right)\right) \mathrm{d} s\right) \\
& \quad \quad+\mathbb{E}\left(\left(\xi^{m}-\xi^{n}\right)^{2}\right) \\
& =2 \mathbb{E}\left(\int_{0}^{T}\left|Y_{s-}^{n}-\left(Y_{s}\right)^{\mathrm{p}}\right|\left(K_{1}+2 \widehat{L}_{s}+K_{2}\left(\left\|Z_{s}^{n}-Z_{s}\right\|^{2}+\left\|Z_{s}\right\|^{2}+\left|U_{s}^{n}-U_{s}\right|_{s}^{2}+\left|U_{s}\right|_{s}^{2}\right)\right) \mathrm{d} s\right) \\
& \quad+\mathbb{E}\left(\left(\xi-\xi^{n}\right)^{2}\right) .
\end{aligned}
$$

Noting $3 / 4-2 K_{2}\left|Y_{s-}^{n}-\left(Y_{s}\right)^{\mathrm{p}}\right| \geq 3 / 4-4 K_{2} \tilde{c} \geq 1 / 2$, one obtains with dominated convergence

$$
\begin{aligned}
& \frac{1}{2} \limsup _{n \rightarrow \infty} \mathbb{E}\left(\int_{0}^{T}\left\|Z_{s}^{n}-Z_{s}\right\|^{2}+\left|U_{s}^{n}-U_{s}\right|_{s}^{2} \mathrm{~d} s\right) \\
& \quad \leq \limsup _{n \rightarrow \infty} 2 \mathbb{E}\left(\int_{0}^{T}\left|Y_{s-}^{n}-\left(Y_{s}\right)^{\mathrm{p}}\right|\left(K_{1}+2 \widehat{L}_{s}+\left\|Z_{s}\right\|^{2}+\left|U_{s}\right|_{s}^{2}\right) \mathrm{d} s\right)+\mathbb{E}\left(\left(\xi^{n}-\xi\right)^{2}\right)=0 .
\end{aligned}
$$

We will need the following result which is a slight variation of [Kob00, Lem.2.5].

Lemma 4.10. Let $\left(Z^{n}\right)_{n \in \mathbb{N}}$ be convergent in $\mathcal{L}^{2}(B)$ and $\left(U^{n}\right)_{n \in \mathbb{N}}$ convergent in $\mathcal{L}^{2}(\widetilde{\mu})$. Then there exists a subsequence $\left(n_{k}\right)_{k \in \mathbb{N}}$ such that $\sup \left\|Z^{n_{k}}\right\| \in L^{2}(\mathbb{P} \otimes \mathrm{d} t)$ and $\sup \left|U_{t}^{n_{k}}\right|_{t} \in L^{2}(\mathbb{P} \otimes \mathrm{d} t)$. 
Proof. The result for $\left(Z^{n}\right)_{n \in \mathbb{N}}$ is from [Kob00] and the argument for $\left(U^{n}\right)_{n \in \mathbb{N}}$ is analogous.

Theorem 4.11 (Monotone stability, infinite activity). Let $\xi \in L^{\infty}\left(\mathcal{F}_{T}\right)$ and let $\left(f^{n}\right)_{n}$ be a sequence of generators satisfying condition $\left(B_{\gamma^{n}}\right)$ with $K_{f}^{y, z}:=\sup _{n \in \mathbb{N}} K_{f^{n}}^{y, z}<\infty$. Assume that

1. there is $(\widehat{Y}, \widehat{Z}, \widehat{U})$ in $\mathcal{S}^{\infty} \times \mathcal{L}^{2}(B) \times \mathcal{L}^{2}(\widetilde{\mu})$ with $\widehat{U}$ bounded and $f_{t}^{n}\left(\widehat{Y}_{t-}, \widehat{Z}_{t}, \widehat{U}_{t}\right) \equiv 0$ for all $n$,

2. for all $u \in L^{0}(\mathcal{B}(E), \lambda)$ with $|u| \leq|\widehat{U}|_{\infty}+2|\xi|_{\infty} \exp \left(K_{f}^{y, z} T\right)$ there exists $\widehat{K} \in \mathbb{R}_{+}$and a process $\widehat{L} \in L^{1}(\mathbb{P} \otimes \mathrm{d} t)$ such that $\left|f_{t}^{n}(0,0, u)\right| \leq \widehat{K}|u|_{t}^{2}+\widehat{L}_{t}$ for each $n \in \mathbb{N}$,

3. the sequence $\left(f^{n}\right)_{n \in \mathbb{N}}$ converges pointwise and monotonically to a generator $f$,

4. there is a $\mathrm{BMO}(\mathbb{P})$-martingale $M$ such that for all truncated generators $f_{t}^{n, \hat{c}}(y, z, u):=$ $f_{t}^{n}((y \vee(-\hat{c})) \wedge \hat{c}, z,(u \vee(-2 \hat{c})) \wedge(2 \hat{c}))$ with $\hat{c}:=|\widehat{Y}|_{\infty}+\left(|\widehat{U}|_{\infty} / 2\right)+\exp \left(K_{f}^{y, z} T\right)|\xi|_{\infty}$ holds $\int_{t}^{T} f_{s}^{n, \hat{c}}\left(Y_{s-}, Z_{s}, U_{s}\right) \mathrm{d} s \leq\langle M\rangle_{T}-\langle M\rangle_{t}$ or $-\int_{t}^{T} f_{s}^{n, \hat{c}}\left(Y_{s-}, Z_{s}, U_{s}\right) \mathrm{d} s \leq\langle M\rangle_{T}-\langle M\rangle_{t}$ for all $n \in \mathbb{N},(Y, Z, U) \in \mathcal{S}^{\infty} \times \mathcal{L}^{2}(B) \times \mathcal{L}^{2}(\widetilde{\mu})$, and

5. for all $(Y, Z, U) \in \mathcal{S}^{\infty} \times \mathcal{L}^{2}(B) \times \mathcal{L}^{2}(\widetilde{\mu})$ and $\left(U^{n}\right)_{n \in \mathbb{N}} \in \mathcal{L}^{2}(\widetilde{\mu})$ with $U^{n} \rightarrow U$ in $L^{2}(\widetilde{\mu})$ it holds $f^{n}\left(Y_{-}, Z, U^{n}\right) \longrightarrow f\left(Y_{-}, Z, U\right)$ in $L^{1}(\mathbb{P} \otimes \mathrm{d} t)$.

Then

i) there exists a solution $(Y, Z, U) \in \mathcal{S}^{\infty} \times \mathcal{L}^{2}(B) \times \mathcal{L}^{2}(\widetilde{\mu})$ for the $B S D E(\xi, f)$, with $\int Z \mathrm{~d} B$ and $U * \widetilde{\mu}$ being $\mathrm{BMO}(\mathbb{P})$-martingales, and

ii) this solution is unique if additionally $f$ satisfies condition $\left(\mathbf{A}_{\gamma}^{\prime}\right)$.

Proof. Let us first outline the overall program of the proof. We want to construct generators $\left(f^{k, n}\right)_{1 \leq k \leq N, n \in \mathbb{N}}$ and solutions $\left(Y^{k, n}, Z^{k, n}, U^{k, n}\right)$ to the BSDEs $\left(\xi / N, f^{k, n}\right)$ for $N$ sufficiently large (to employ Proposition 4.9 and get that $\left(\left(Y^{k, n}, Z^{k, n}, U^{k, n}\right)\right)_{n \in \mathbb{N}}$ converges and $\left(Y^{n}, Z^{n}, U^{n}\right):=$ $\sum_{k=1}^{N}\left(Y^{k, n}, Z^{k, n}, U^{k, n}\right)$ solves the $\left.\operatorname{BSDE}\left(\xi, f^{n}\right)\right)$. We show that if for some $k<N$ and all $1 \leq l \leq k$ and $n \in \mathbb{N}$ we have already constructed generators $\left(f^{l, n}\right)_{1 \leq l \leq k, n \in \mathbb{N}}$ such that there exists solutions $\left(\left(Y^{l, n}, Z^{l, n}, U^{l, n}\right)\right)_{n \in \mathbb{N}}$ to the BSDEs $\left(\xi / N, f^{l, n}\right)$ converging for $n \rightarrow \infty$, with $\left|Y^{l, n}\right|_{\infty} \leq \exp \left(K_{f}^{y, z} T\right)|\xi|_{\infty} / N=: \tilde{c}$, then for $\bar{Y}^{k, n}:=\widehat{Y}+\sum_{l=1}^{k} Y^{l, n}$ with $\bar{Z}^{k, n}$ and $\bar{U}^{k, n}$ defined analogously and

$$
f_{t}^{k+1, n}(y, z, u):=f_{t}^{n}\left(y+\bar{Y}_{t-}^{k, n}, z+\bar{Z}_{t}^{k, n}, u+\bar{U}_{t}^{k, n}\right)-f_{t}^{n}\left(\bar{Y}_{t-}^{k, n}, \bar{Z}_{t}^{k, n}, \bar{U}_{t}^{k, n}\right)
$$

there are solutions $\left(Y^{k+1, n}, Z^{k+1, n}, U^{k+1, n}\right) \in \mathcal{S}^{\infty} \times \mathcal{L}^{2}(B) \times \mathcal{L}^{2}(\widetilde{\mu})$ to the BSDEs $\left(\xi / N, f^{k+1, n}\right)$, converging (in $n$ ) and satisfying $\left|Y^{k+1, n}\right|_{\infty} \leq \tilde{c}$. Starting initially with the triple $\left(Y^{0, n}, Z^{0, n}, U^{0, n}\right)$ defined by $\left(Y^{0, n}, Z^{0, n}, U^{0, n}\right):=(\widehat{Y}, \widehat{Z}, \widehat{U})$, formula $(4.3)$ gives an inductive construction of the generators $f^{k, n}$ and triples $\left(Y^{k, n}, Z^{k, n}, U^{k, n}\right) \in \mathcal{S}^{\infty} \times \mathcal{L}^{2}(B) \times \mathcal{L}^{2}(\widetilde{\mu})$ solving the $\operatorname{BSDE}\left(\xi / N, f^{k, n}\right)$ and converging for $n \rightarrow \infty$ with $\left|Y^{k, n}\right|_{\infty} \leq \tilde{c}$ for each $n \in N$ and $1 \leq k \leq N$.

Note that $f^{k+1, n}$ is Lipschitz continuous in $y$ and $z$ with Lipschitz constant $K_{f^{n}}^{y, z}$, locally Lipschitz in $u$ and satisfies condition $\left(A_{\gamma^{k+1, n}}\right)$ with

$$
\gamma_{s}^{k+1, n}\left(y, z, u, u^{\prime}, e\right):=\gamma_{s}^{n}\left(y+\bar{Y}_{s-}^{k, n}, z+\bar{Z}_{s}^{k, n}, u+\bar{U}_{s}^{k, n}(e), u^{\prime}+\bar{U}_{s}^{k, n}(e), e\right)
$$

and $f_{t}^{k+1, n}(0,0,0) \equiv 0$. Hence by the existence and uniqueness result for the finite activity case (see Proposition 4.2), there exists a unique solution $\left(Y^{k+1, n}, Z^{k+1, n}, U^{k+1, n}\right)$ to the BSDE $\left(\xi / N, f^{k+1, n}\right)$ such that $Y^{k+1, n}$ is bounded by $\tilde{c}$.

To apply Proposition 4.9 , we have to check that the sequence $\left(Y^{k+1, n}\right)_{n \in \mathbb{N}}$ converges pointwise, that $\left(Z^{k+1, n}, U^{k+1, n}\right)_{n \in \mathbb{N}}$ converges weakly in $\mathcal{L}^{2}(B) \times \mathcal{L}^{2}(\widetilde{\mu})$ and that $f^{k+1, n}(0,0, u)$ can be locally bounded by an affine function in $|u|^{2}$. Having telescoping sums in (4.3) implies that $\left(\bar{Y}^{l, n}, \bar{Z}^{l, n}, \bar{U}^{l, n}\right)$ solves the BSDE $\left(\widehat{Y}_{T}+l \xi / N, f^{n}\right)$. By the comparison result of Proposition 3.1, the sequences $\left(\bar{Y}^{k, n}\right)_{n \in \mathbb{N}}$ and $\left(\bar{Y}^{k+1, n}\right)_{n \in \mathbb{N}}$ are monotonic (and bounded) in $n$ so that finite limits $\lim _{n \rightarrow \infty} Y^{k+1, n}=\lim _{n \rightarrow \infty} \bar{Y}^{k+1, n}-\lim _{n \rightarrow \infty} \bar{Y}^{k, n}$ exists, $\mathbb{P} \otimes \mathrm{d} t$-a.e.. By Lemma 2.3, the sequences 
$\left(\bar{Z}^{k, n}, \bar{U}^{k, n}\right)_{n \in \mathbb{N}}$ and $\left(\bar{Z}^{k+1, n}, \bar{U}^{k+1, n}\right)_{n \in \mathbb{N}}$ are bounded in $\mathcal{L}^{2}(B) \times \mathcal{L}^{2}(\widetilde{\mu})$; hence $\left(Z^{k+1, n}, U^{k+1, n}\right)$ is weakly convergent in $\mathcal{L}^{2}(B) \times \mathcal{L}^{2}(\widetilde{\mu})$ along a subsequence which we still index by $n$ for simplicity. Due to the Lipschitz continuity of $f^{n}$ and Assumption 2., we get for all $|u| \leq 2 \tilde{c}$ that

$$
\begin{aligned}
\left|f_{t}^{k+1, n}(0,0, u)\right| & \leq\left|f_{t}^{n}\left(\bar{Y}_{t-}^{k, n}, \bar{Z}_{t}^{k, n}, u+\bar{U}_{t}^{k, n}\right)-f_{t}^{n}\left(\bar{Y}_{t-}^{k, n}, \bar{Z}_{t}^{k, n}, \bar{U}_{t}^{k, n}\right)\right| \\
& \leq 2 K_{f^{n}}^{y, z}\left(\left|\bar{Y}_{t-}^{k, n}\right|+\left\|\bar{Z}_{t}^{k, n}\right\|\right)+\widehat{K}\left(\left|u+\bar{U}_{t}^{k, n}\right|_{t}^{2}+\left|\bar{U}_{t}^{k, n}\right|_{t}^{2}\right)+2 \widehat{L}_{t} \\
& \leq 2 \widehat{K}|u|_{t}^{2}+\widetilde{L}_{t},
\end{aligned}
$$

where $\widetilde{L}_{t}=2 K_{f}^{y, z}\left(\hat{c}+\sup _{n \in \mathbb{N}}\left\|\bar{Z}_{t}^{k, n}\right\|^{2}+1 / 4\right)+3 \widehat{K} \sup _{n \in \mathbb{N}}\left|\bar{U}_{t}^{k, n}\right|_{t}^{2}+2 \widehat{L}_{t}$. Here we used that by induction hypothesis $\left(\bar{Z}^{k, n}, \bar{U}^{k, n}\right)_{n}$ is convergent so that $\sup _{n \in \mathbb{N}}\left(\left\|\bar{Z}_{t}^{k, n}\right\|^{2}+\left|\bar{U}_{t}^{k, n}\right|_{t}^{2}\right)$ is $\mathbb{P} \otimes \mathrm{d} t$-integrable by Lemma 4.10 along a subsequence which again for simplicity we still index by $n$. This implies that $\widetilde{L} \in L^{1}(\mathbb{P} \otimes \mathrm{d} t)$, and therefore by Proposition 4.9 , the sequence $\left(Z^{n}, U^{n}\right):=\left(\bar{Z}^{N, n}, \bar{U}^{N, n}\right)$ converges in $\mathcal{L}^{2}(B) \times \mathcal{L}^{2}(\widetilde{\mu})$ to some $(Z, U)$ in $\mathcal{L}^{2}(B) \times \mathcal{L}^{2}(\widetilde{\mu})$ while $\left(Y^{n}\right):=\left(\bar{Y}^{N, n}\right)$ converges to some $Y$. Hence, $f^{n}\left(Y_{-}^{n}, Z^{n}, U^{n}\right)-f^{n}\left(Y_{-}, Z, U^{n}\right)$ converges to 0 in $L^{1}(\mathbb{P} \otimes \mathrm{d} t)$ and by Assumption 5. we have $f^{n}\left(Y_{-}^{n}, Z^{n}, U^{n}\right) \rightarrow f\left(Y_{-}, Z, U\right)$ in $L^{1}(\mathbb{P} \otimes \mathrm{d} t)$. The stochastic integrals $\left(Z^{n}-Z^{m}\right) \cdot B$ and $\left(U^{n}-U^{m}\right) * \widetilde{\mu}$ belong to $\mathcal{S}^{2} \subset \mathcal{S}^{1}$ by Doob's inequality, with $\mathcal{S}^{1}$-norms being bounded by a multiple of $\left\|Z^{n}-Z^{m}\right\|_{\mathcal{L}^{2}(B)}$ and $\left\|U^{n}-U^{m}\right\|_{\mathcal{L}^{2}(\widetilde{\mu})}$ respectively. Since $\left|Y^{n}-Y^{m}\right|_{\mathcal{S}^{1}}$ is dominated by

$$
\left\|f^{n}\left(Y_{-}^{n}, Z^{n}, U^{n}\right)-f^{m}\left(Y_{-}^{m}, Z^{m}, U^{m}\right)\right\|_{L^{1}(\mathbb{P} \otimes \mathrm{d} t)}+C\left(\left\|Z^{n}-Z^{m}\right\|_{\mathcal{L}^{2}(B)}+\left\|U^{n}-U^{m}\right\|_{\mathcal{L}^{2}(\widetilde{\mu})}\right)
$$

for some constant $C>0$ with the bound tending to 0 as $n, m \rightarrow 0$, we can take $Y$ in $\mathcal{S}^{1}$ due to completeness of $\mathcal{S}^{1}$; see [DM82, VII. 3,64].

Finally, $(Y, Z, U)$ solves the $\operatorname{BSDE}(\xi, f)$ since the approximating solutions $\left(Y^{n}, Z^{n}, U^{n}\right)_{n \in \mathbb{N}}$ of the $\operatorname{BSDE}\left(\xi, f^{n}\right)_{n \in \mathbb{N}}$ converge to some $(Y, Z, U) \in \mathcal{S}^{\infty} \times \mathcal{L}^{2}(B) \times \mathcal{L}^{2}(\widetilde{\mu})$ and $f^{n}\left(Y_{-}^{n}, Z^{n}, U^{n}\right)$ tends to $f\left(Y_{-}, Z, U\right)$ in $L^{1}(\mathbb{P} \otimes \mathrm{d} t)$. Hence, we have $\int_{0}^{t} f_{s}^{n}\left(Y_{s-}^{n}, Z_{s}^{n}, U_{s}^{n}\right) \mathrm{d} s \rightarrow \int_{0}^{t} f_{s}\left(Y_{s-}, Z_{s}, U_{s}\right) \mathrm{d} s$, $\int_{0}^{t} Z_{s}^{n} \mathrm{~d} B_{s} \rightarrow \int_{0}^{t} Z_{s} \mathrm{~d} B_{s}$ and $U^{n} * \widetilde{\mu}_{t} \rightarrow U * \widetilde{\mu}_{t} \mathbb{P}$-a.s. (along a subsequence) for all $0 \leq t \leq T$.

The next corollary to Theorem 4.11 provides conditions under which the $Z$-component of the JBSDE solution vanishes. Such is useful for applications in a pure-jump context (see e.g. Section 5.1.2 or [CFJ16]) with weak PRP by $\widetilde{\mu}$ alone (cf. Example 2.1, Parts 1.,3.,4.), without a Brownian motion. Clearly an independent Brownian motion can always be added by enlarging the probability space, but it is then natural to ask for a JBSDE solution with trivial $Z$-component, adapted to the original filtration. Instead of re-doing the entire argument leading to Theorem 4.11 but now for JBSDEs solely driven by a random measure $\widetilde{\mu}$ with generators without a $z$-argument, the next result gives a direct argument to this end. An example where the corollary is applied is given in Section 5.1.2.

Corollary 4.12. Let $\mu=\mu^{X}$ be the random measure associated to a pure-jump process $X$, such that the compensated random measure $\widetilde{\mu}$ alone has the weak PRP (see (2.2)) with respect to the usual filtration $\mathbb{F}^{X}$ of $X$. Let $B$ be a d-dimensional Brownian motion independent of $X$. With respect to $\mathbb{F}:=\mathbb{F}^{B, X}$, let $f,\left(f^{n}\right)_{n}, \xi$ satisfy the assumptions of Theorem 4.11 with $\widehat{Z}=0$ and $f$ satisfying $\left(\mathbf{A}_{\gamma}^{\prime}\right)$. Let $\xi$ be in $L^{\infty}\left(\mathcal{F}_{T}^{X}\right)$ and $f, f^{n}$ be $\mathcal{P}\left(\mathbb{F}^{X}\right) \otimes \mathcal{B}\left(\mathbb{R}^{d+1}\right) \otimes \mathcal{B}\left(L^{0}(\mathcal{B}(E))\right)$-measurable. Then the JBSDE $(\xi, f)$ admits a unique solution $(Y, Z, U)$ in $\mathcal{S}^{\infty} \times \mathcal{L}^{2}(B) \times \mathcal{L}^{2}(\widetilde{\mu})$, and we have that $Y$ is $\mathbb{F}^{X}$-adapted, $Z=0$, and $U$ can be taken as measurable with respect to $\widetilde{\mathcal{P}}\left(\mathbb{F}^{X}\right)$.

Proof. Let $B^{\prime}$ be a (1-dimensional) Brownian motion independent of $(B, X)$. Then $\bar{B}:=\left(B, B^{\prime}\right)$ is a $(d+1)$-dimensional Brownian motion independent of $X$. Let $\mathbb{F}^{\prime}:=\mathbb{F}^{B^{\prime}, X}$ and $\overline{\mathbb{F}}:=\mathbb{F}^{\bar{B}, X}$ denote the usual filtrations of $\left(B^{\prime}, X\right)$ and $(\bar{B}, X)$. As in Example 2.1.3., $(B, \widetilde{\mu}),\left(B^{\prime}, \widetilde{\mu}\right)$ and $(\bar{B}, \widetilde{\mu})$ each admits the weak PRP w.r.t. $\mathbb{F}, \mathbb{F}^{\prime}$ and $\overline{\mathbb{F}}$ respectively. Now consider the generator function $\widetilde{f}$ that does not depend on $z$ and is defined by $\widetilde{f}_{t}(y, u):=f_{t}(y, 0, u)$. Because $\widehat{Z}=0$, the conditions for Theorem 4.11 are met by $\widetilde{f}^{n}:=f^{n}(\cdot, 0, \cdot)$. In addition, $\widetilde{f}$ satisfies condition $\left(\mathbf{A}_{\gamma}^{\prime}\right)$ since $f$ does. Since $\xi$ is $\mathcal{F}_{T}^{X}$-measurable and $\widetilde{f}$ is $\mathcal{P}\left(\mathbb{F}^{X}\right) \otimes \mathcal{B}(\mathbb{R}) \otimes \mathcal{B}\left(L^{0}(\mathcal{B}(E))\right)$-measurable, then by Theorem 4.11 the JBSDE $(\xi, \widetilde{f})$ simultaneously admits unique solutions $(Y, Z, U),\left(Y^{\prime}, Z^{\prime}, U^{\prime}\right)$ and $(\bar{Y}, \bar{Z}, \bar{U})$ in the respective $\mathcal{S}^{\infty} \times \mathcal{L}^{2}(\cdot) \times \mathcal{L}^{2}(\widetilde{\mu})$-spaces for each of the filtrations $\mathbb{F}, \mathbb{F}^{\prime}$ and $\overline{\mathbb{F}}$. Noting that both 
$\mathbb{F}$ and $\mathbb{F}^{\prime}$ are sub-filtrations of $\overline{\mathbb{F}}$, we get by uniqueness of $(\bar{Y}, \bar{Z}, \bar{U})$ that $Z \cdot B=Z^{\prime} \cdot B^{\prime}=\bar{Z} \cdot \bar{B}$ and that $Y$ is $\mathbb{F}^{X}$-adapted. The former implies $Z=Z^{\prime}=0$ by the strong orthogonality of $B$ and $B^{\prime}$. The claim follows, by noting that the JBSDE gives the (unique) canonical decomposition of the special semimartingale $Y$ and using weak predictable martingale representation in $\mathbb{F}^{X}$.

A natural ansatz to approximate an $f$ of the form $(2.7)$ with $\lambda(A)=\infty$ is by taking

$$
f_{t}^{n}(y, z, u):=\widehat{f}_{t}(y, z)+\int_{A^{n}} g_{t}(u(e), e) \zeta(t, e) \lambda(\mathrm{d} e),
$$

for an increasing sequence $\left(A_{n}\right)_{n \in \mathbb{N}} \uparrow A$ of measurable sets with $\lambda\left(A_{n}\right)<\infty$ (as $\lambda$ is $\sigma$-finite).

Theorem 4.13 (Wellposedness, infinite activity of jumps). Let the generator $f$ of the JBSDE be of the form (2.7) and let $\xi$ be in $L^{\infty}\left(\mathcal{F}_{T}\right)$. Let $\widehat{f}$ be Lipschitz continuous with respect to $(y, z)$ uniformly in $(\omega, t, u)$, and let $u \mapsto g(t, u, e)$ be absolutely continuous in $u$, for all $(\omega, t, e)$, with its density function $g^{\prime}(t, u, e)$ being strictly greater than -1 and locally bounded (in u) from above.

Assume that

1. there exists $(\widehat{Y}, \widehat{Z}, \widehat{U}) \in \mathcal{S}^{\infty} \times \mathcal{L}^{2}(B) \times \mathcal{L}^{2}(\widetilde{\mu})$ with $|\widehat{U}|_{\infty}<\infty, \widehat{f}_{t}\left(\widehat{Y}_{t}, \widehat{Z}_{t}\right) \equiv 0, g_{t}\left(\widehat{U}_{t}(e), e\right) \equiv 0$,

2. the function $g$ is locally bounded in $|u|^{2}$ uniformly in $(\omega, t, e)$, i.e. locally in $u$ (for any bounded neighborhood $N$ of 0 ) there exists a $K>0$ such that $\left|g_{t}(u, e)\right| \leq K|u|^{2}$ (for all $u \in N$ ),

3. and there exists $D: \mathbb{R} \mapsto \mathbb{R}$ continuous such that either $g \geq 0$ and $\widehat{f}_{t}(y, z) \geq D(y)$ for $|y| \leq \hat{c}:=|\widehat{Y}|_{\infty}+\left(|\widehat{U}|_{\infty} / 2\right)+|\xi|_{\infty} \exp \left(K_{\widehat{f}}^{y, z} T\right)$, or $g \leq 0$ and $\widehat{f}_{t}(y, z) \leq D(y)$ for $|y| \leq \hat{c}$.

Then

i) there exists a solution $(Y, Z, U) \in \mathcal{S}^{\infty} \times \mathcal{L}^{2}(B) \times \mathcal{L}^{2}(\widetilde{\mu})$ to the JBSDE and for each solution triple the stochastic integrals $\int Z \mathrm{~d} B$ and $U * \widetilde{\mu}$ are BMO-martingales, and

ii) this solution is unique if moreover the function $g$ satisfies condition $\left(\mathbf{A}_{\mathbf{i n f}}\right)$.

Finally, the same statements hold if condition 1 . is replaced by assuming that $f$ is not depending on $y$, i.e. $f_{t}(y, z, u)=f_{t}(z, u)$, and that $\widehat{f}$ is bounded.

Proof. We check that the assumptions of Theorem 4.11 are satisfied. Clearly conditions 1 . and 2. are sufficient for assumptions 1 . and 2. in Theorem 4.11. The $f^{n}$ given by (4.4) satisfy conditions $\left(B_{\gamma^{n}}\right)$ (cf. Example 3.8 and note $\lambda\left(A_{n}\right)<\infty$ ) and the sequence $\left(f^{n}\right)$ is either monotone increasing or monotone decreasing, depending on the sign of $g$. For the next assumption $4 ., f^{n, \hat{c}}$ is bounded from above (or resp. below) by $\sup _{|y| \leq \hat{c}} D(y)$ (respectively $\inf _{|y| \leq \hat{c}} D(y)$ ). To show that also condition 5. of Theorem 4.11 holds, we prove that $g_{t}\left(U_{t}^{n}(e), e\right) \mathbb{1}_{A_{n}}(e)$ converge to $g_{t}\left(U_{t}(e), e\right)$ in $L^{1}(\mathbb{P} \otimes \nu)$ as $n \rightarrow \infty$ for $U^{n} \rightarrow U$ in $\mathcal{L}^{2}(\widetilde{\mu})$, recalling (2.1). We set $B_{n}:=\left(g_{t}\left(U_{t}^{n}(e), e\right)-g_{t}\left(U_{t}(e), e\right)\right) \mathbb{1}_{A_{n}}(e)$ and $C_{n}:=g_{t}\left(U_{t}(e), e\right) \mathbb{1}_{A_{n}^{c}}(e)$. Both sequences $\left(B_{n}\right)_{n \in \mathbb{N}}$ and $\left(C_{n}\right)_{n \in \mathbb{N}}$ converge to $0 \mathbb{P} \otimes \nu$-a.e. since $U^{n} \rightarrow U$ in $L^{2}(\mathbb{P} \otimes \nu), g$ is locally Lipschitz in $u$ and $A_{n}^{c} \downarrow \emptyset$. Moreover, they are bounded by integrable random variables. In particular, $B_{n}$ is bounded by $\widehat{K}\left(\sup _{n \in \mathbb{N}}\left|U_{t}^{n}(e)\right|^{2}+\left|U_{t}(e)\right|^{2}\right)$ for some $\widehat{K}>0$ which is integrable along a subsequence due to Lemma 4.10. Hence applying the dominated convergence theorem yields the desired result.

In the alternative case without the Assumption 1., existence is still guaranteed. Indeed, let $f_{t}(y, z, u)=f_{t}(z, u)$ and $\widehat{f}$ be bounded. Denoting $\widetilde{f}_{t}(z, u):=f_{t}(z, u)-f_{t}(0,0)$ and $\widetilde{\xi}:=\xi+$ $\int_{0}^{T} f_{t}(0,0) \mathrm{d} t$, there exists a unique solution $(\widetilde{Y}, Z, U)$ in $\mathcal{S}^{\infty} \times \mathcal{L}^{2}(B) \times \mathcal{L}^{2}(\widetilde{\mu})$ to the BSDE $(\widetilde{\xi}, \widetilde{f})$ with $\int Z \mathrm{~d} B$ and $U * \widetilde{\mu}$ being BMO-martingales by the first version of this theorem and noting that $g_{t}(0, e) \equiv 0$ and $f_{t}(0,0)=\widehat{f}_{t}(0)$ is bounded. Taking $Y_{t}:=\widetilde{Y}_{t}-\int_{0}^{t} \bar{f}_{s}(0,0) \mathrm{d} s$, we obtain that $(Y, Z, U)$ solves the BSDE with the data $(\xi, f)$. If moreover the function $f$ satisfies $\left(\mathbf{A}_{\text {infi }}\right)$, then $f$ satisfies $\left(\mathbf{A}_{\gamma}^{\prime}\right)$ (cf. Example 3.8.3.) and hence uniqueness follows from applicability of the comparison argument in Proposition 3.1.

Example 4.14. A function $g$ is locally bounded in $|u|^{2}$ in the sense of condition 2. in Theorem 4.13 if, for instance, $u \mapsto g_{t}(u, e)$ is twice differentiable for any $(\omega, t, e)$, with the second derivative in $u$ being locally bounded uniformly in $(\omega, t, e)$, and $g_{t}(0, e) \equiv g_{t}^{\prime}(0, e) \equiv 0$ vanishing. 
Example 4.15. An example for a generator that satisfies the assumption of Theorem 4.13 but has super-exponential growth is $f$ of the form (2.7) with $\widehat{f} \equiv 0$ and $g_{t}(u)=\exp \left(\left|u^{+}\right|^{2}\right)-1$. Here exists, in general, no $\gamma \in(0, \infty)$ such that $-\frac{1}{\gamma}\left(e^{-u / \gamma}+u / \gamma-1\right) \leq g_{t}(u) \leq \frac{1}{\gamma}\left(e^{u / \gamma}-u / \gamma-1\right)$ holds for all $u$ and $t$. Thus, the example appears not to satisfy exponential growth assumptions as formulated, e.g., in [AM16][Assumption (H), Thm.1], [EMN16][2.condition, Def.5.6] or [KTPZ15][Assumption 3.1].

Note that convexity is not required for our theorems on comparison, existence and uniqueness for JBSDEs. Many relevant applications are convex in nature but not all, see examples in Section 5.1.2.

\section{Examples and applications: optimal control in finance}

Results for JBSDEs in the literature commonly rely on combinations of several, often quite technical, assumptions. But their scope can be difficult to judge at first sight without examples, and to verify them may be not easy. This section discusses key applications that JBDEs have found in mathematical finance, and it illustrates by concrete examples the applicability and the scope of the theory from previous sections. The examples do also help to shed some light on connections and differences to related literature. Counter examples might caution against potential pitfalls.

The applications in Section 5.1 are about exponential utility maximization, possibly with an additive liability or non-convex constraints. This problem is closely related to the entropic risk measure and to (exponential) utility indifference valuation; It has indeed been a standard motivation for much of the (quadratic, non-Lipschitz) JBSDE theory, cf. [Bec06, BEK09, Mor09, Bec10, LS14, KTPZ15]. A result on existence of a solution for the specific JBSDE of this application has been presented in [Mor10], being more general in some aspects (jump-diffusion stock price) but less so in others (multiple assets, time-inhomogeneous $\mu$ ). Section 5.2 shows how a change of coordinates can transform a JBSDE, which arises from an optimal control problem for power utility maximization but appears to be out of scope at first, into a JBSDE for which theory of Section 4 can be applied to derive optimal controls and fully characterize the solution to the control problem by JBSDE solutions, like in [HIM05, HLT17], by using martingale optimality principles. To our best knowledge, the considered power-utility problem with jumps and a multiplicative liability is solved for the first time in this spirit. Finally, Section 5.3 derives JBSDE solutions for the no-good-deal valuation problem in incomplete markets, which is posed over a multiplicatively stable sub-family of arbitrage-free pricing measures. Also here, where the (non-linear) JBSDE generator is even Lipschitz, the slight generalization of Proposition 3.1 to the classical comparison result by [Roy06] is useful; Indeed, the process $\gamma$ in (5.17) is such that the martingale condition (3.1) for Proposition 3.1 can be readily verified, while the same appears not clear for condition $\left(\mathbf{A}_{\boldsymbol{\gamma}}\right)$ in [Roy06, Thm.2.3].

Sections 5.1.1, 5.2 and 5.3 consider models for a financial market within the framework of Section 2, consisting of one savings account with zero interest rate (for simplicity) and $k$ risky assets $(k \leq d)$, whose discounted prices evolve according to the stochastic differential equation

$$
\mathrm{d} S_{t}=\operatorname{diag}\left(S_{t}^{i}\right)_{1 \leq i \leq k} \sigma_{t}\left(\varphi_{t} \mathrm{~d} t+\mathrm{d} B_{t}\right)=: \operatorname{diag}\left(S_{t}\right) \mathrm{d} R_{t}, \quad t \in[0, T],
$$

with $S_{0} \in(0, \infty)^{k}$, where the market price of risk $\varphi$ is a predictable $\mathbb{R}^{d}$-valued process, with $\varphi_{t} \in \operatorname{Im} \sigma_{t}^{T}=\left(\operatorname{Ker} \sigma_{t}\right)^{\perp}$ for all $t \leq T$, and $\sigma$ is a predictable $\mathbb{R}^{k \times d}$-valued process such that $\sigma$ is of full rank $k$ (i.e. $\operatorname{det}\left(\sigma_{t} \sigma_{t}^{T}\right) \neq 0 \mathbb{P} \otimes \mathrm{d} t$-a.e.) and integrable w.r.t. $\widehat{B}:=B+\int_{0}^{\cdot} \varphi_{t} \mathrm{~d} t$. We take the market price of risk $\varphi$ to be bounded $\mathbb{P} \otimes \mathrm{d} t$-a.e.. The market is free of arbitrage in the sense that the set $\mathcal{M}^{\mathrm{e}}$ of equivalent local martingale measures for $S$ is non-empty. In particular, $\mathcal{M}^{\mathrm{e}}$ contains the minimal martingale measure

$$
\mathrm{d} \widehat{\mathbb{P}}:=\mathcal{E}(-\varphi \cdot B)_{T} \mathrm{~d} \mathbb{P}=\exp \left(-\varphi \cdot B_{T}-\frac{1}{2} \int_{0}^{T}\left|\varphi_{t}\right|^{2} \mathrm{~d} t\right) \mathrm{d} \mathbb{P}
$$

under which $\widehat{B}$ is a Brownian motion and $S$ is a local martingale by Girsanov's theorem. Clearly, the market (5.1) is incomplete in general (even if $k=d$ and $\sigma$ is invertible, when the random measure is not trivial, filtration then being non-Brownian), cf. Example 2.1. 


\subsection{Exponential utility maximization}

For a market with stock prices as in (5.1), consider the expected utility maximization problem

$$
v_{t}(x)=\underset{\theta \in \Theta}{\operatorname{ess} \sup _{0}} \mathbb{E}\left(u\left(X_{T}^{\theta, t, x}-\xi\right) \mid \mathcal{F}_{t}\right), \quad t \leq T, x \in \mathbb{R},
$$

for the exponential utility function $u(x):=-\exp (-\alpha x)$ with absolute risk aversion parameter $\alpha>0$, with some additive liability $\xi$ and for wealth processes $X^{\theta, t, x}$ of admissible trading strategies $\theta$ as defined below. We are going to show, how the value process $v$ and optimal trading strategy $\theta^{*}$ for the problem (5.3) can be fully described by JBSDE solutions for two distinct problem cases.

\subsubsection{Case with continuous price processes of risky assets}

The set of available trading strategies $\Theta$ consists of all $\mathbb{R}^{d}$-valued, predictable, S-integrable processes $\theta$ for which the following two conditions are satisfied: $\mathbb{E}\left(\int_{0}^{T}\left|\theta_{t}\right|^{2} \mathrm{~d} t\right)$ is finite, and the family $\left\{\exp \left(-\alpha \int_{0}^{\tau} \theta_{t} \mathrm{~d} \widehat{B}_{t}\right) \mid \tau\right.$ stopping time, $\left.\tau \leq T\right\}$ of random variables is uniformly integrable under $\mathbb{P}$. Starting from initial capital $x \in \mathbb{R}$ at some time $t \leq T$, the wealth process corresponding to investment strategy $\theta \in \Theta$ is given by $X_{s}^{\theta}=X_{s}^{\theta, t, x}=x+\int_{t}^{s} \theta_{u} \mathrm{~d} \widehat{B}_{u}, s \in[t, T]$.

For this subsection, we assume $k=d$ (so $f$ will not be quadratic in $z$ ). Let $(Y, Z, U$ ) in $\mathcal{S}_{\widehat{\mathbb{P}}}^{\infty} \times \mathcal{L}_{\widehat{\mathbb{P}}}^{2}(\widehat{B}) \times \mathcal{L}_{\widehat{\mathbb{P}}}^{2}(\widetilde{\mu})$ be the unique solution to the $\operatorname{BSDE} Y_{t}=\xi+\int_{t}^{T} f_{s}\left(Y_{s-}, Z_{s}, U_{s}\right) \mathrm{d} s-$ $\int_{t}^{T} Z_{s} \mathrm{~d} \widehat{B}_{s}-\int_{t}^{T} \int_{E} U_{s}(e) \widetilde{\mu}(\mathrm{d} s, \mathrm{~d} e)$ under the minimal local martingale measure $\widehat{\mathbb{P}}$ for the generator

$$
f_{t}(y, z, u):=-\frac{\left|\varphi_{t}\right|^{2}}{2 \alpha}+\int_{E} \frac{\exp (\alpha u(e))-\alpha u(e)-1}{\alpha} \zeta(t, e) \lambda(\mathrm{d} e)
$$

which does exist by Theorem 4.13. Under $\mathbb{P}$ the BSDE is of the form

$$
Y_{t}=\xi+\int_{t}^{T} f_{s}\left(Y_{s-}, Z_{s}, U_{s}\right)-\varphi_{s} Z_{s} \mathrm{~d} s-\int_{t}^{T} Z_{s} \mathrm{~d} B_{s}-\int_{t}^{T} \int_{E} U_{s}(e) \widetilde{\mu}(\mathrm{d} s, \mathrm{~d} e) .
$$

To prove optimality by a martingale principle one constructs, cf. [HIM05], a family of processes $\left(V^{\theta}\right)_{\theta \in \Theta}$ such that three conditions are satisfied: (i) $V_{t}^{\theta}=V_{t}$ is a fixed $\mathcal{F}_{t}$-measurable bounded random variable invariant over $\theta \in \Theta$, (ii) $V_{T}^{\theta}=-\exp \left(-\alpha\left(X_{T}^{\theta}-\xi\right)\right)=-\exp \left(-\alpha\left(x+\int_{t}^{T} \theta_{s} \mathrm{~d} \widehat{B}_{s}-\xi\right)\right)$, and (iii) $V^{\theta}$ is a supermartingale for all $\theta \in \Theta$ and there exists a $\theta^{*} \in \Theta$ such that $V_{s}^{\theta^{*}}(s \in[t, T])$ is a $\mathbb{P}$-martingale. Then $\theta^{*}$ is the optimal strategy and $\left(V_{s}^{\theta^{*}}\right)_{s \in[t, T]}$ is the value process of the control problem (5.3). Indeed, $\mathbb{E}\left(V_{T}^{\theta} \mid \mathcal{F}_{t}\right) \leq V_{t}^{\theta}=V_{t}^{\theta^{*}}=\mathbb{E}\left(V_{T}^{\theta^{*}} \mid \mathcal{F}_{t}\right)$ for each $\theta \in \Theta$ implies $v_{t}(x)=\operatorname{ess}_{\sup _{\theta \in \Theta}} \mathbb{E}\left(V_{T}^{\theta} \mid \mathcal{F}_{t}\right)=V_{t}^{\theta^{*}}$. An ansatz $V^{\theta}=u\left(X^{\theta}-Y\right)$ yields

$$
\begin{aligned}
& V_{s}^{\theta}=V_{t}^{\theta} \exp \left(\frac{\alpha^{2}}{2} \int_{t}^{s}\left|\theta_{r}-Z_{r}-\frac{\varphi_{r}}{\alpha}\right|^{2} \mathrm{~d} r\right) \mathcal{E}(M)_{t}^{s} \quad \text { for all } s \in[t, T], \text { with } \\
& M_{t}=-\alpha \int_{0}^{t} \theta_{r}-Z_{r} \mathrm{~d} \widehat{B}_{r}+\int_{0}^{t} \int_{E} \exp \left(\alpha U_{r}(e)-1\right) \tilde{\mu}(\mathrm{d} r, \mathrm{~d} e) \text { and } \mathcal{E}(M)_{t}^{s}:=\frac{\mathcal{E}(M)_{s}}{\mathcal{E}(M)_{t}} .
\end{aligned}
$$

Therefore, $V^{\theta}$ is a supermartingale for all $\theta \in \Theta$ and a martingale for $\theta^{*}=Z+\varphi / \alpha$ due to the fact that $\mathcal{E}(M)$ is a (local) martingale of the form

$$
\mathcal{E}(M)_{s}=\exp \left(-\frac{\alpha^{2}}{2} \int_{0}^{s}\left|\theta_{u}-Z_{u}-\varphi_{u} / \alpha\right|^{2} \mathrm{~d} u\right) \exp \left(-\alpha\left(Y_{0}+\int_{0}^{s} \theta_{u} \mathrm{~d} \widehat{B}_{u}-Y_{s}\right)\right) .
$$

Using the boundedness of $Y$, one readily obtains by arguments like in [HIM05, Mor10] that $\mathcal{E}(M)$ is uniformly integrable and hence a martingale (see e.g. eqn. (4.19) in [Bec06]). This yields

Example 5.1. Let $k=d$ and $\lambda(E) \leq \infty$. Let $(Y, Z, U) \in \mathcal{S}_{\widehat{\mathbb{P}}}^{\infty} \times \mathcal{L}_{\widehat{\mathbb{P}}}^{2}(\widehat{B}) \times \mathcal{L}_{\widehat{\mathbb{P}}}^{2}(\widetilde{\mu})$ be the unique solution to the $B S D E(\xi, f)$ under $\widehat{\mathbb{P}}$ for generator $f$ from (5.4). Then the strategy $\theta^{*}=Z+\varphi / \alpha$ is optimal for the control problem (5.3) and achieves at any time $t \leq T$ the maximal expected exponential utility $v_{t}(x)=-\exp \left(-\alpha\left(x-Y_{t}\right)\right)=V_{t}^{\theta^{*}}$. 
The exponential utility maximization problem is closely linked to the popular entropic convex risk measure, to which we will further relate in Example 5.3. Moreover, the solution to the utility maximization problem is intimately linked to the indifference valuation (also known as reservation price or compensating variation in economics) for a contingent claim $\xi$ in incomplete markets under exponential utility preferences, see [Bec10]. Indeed, denoting by $Y^{\xi}=Y$ the solution to the JBSDE from Example 5.1 for terminal data $\xi$, one can show that $Y^{\xi}-Y^{0}$ yields the utility indifference valuation process, see [MS05, Bec06].

\subsubsection{Case with discontinuous risky asset price processes}

We further illustrate the extend to which results by [Mor09, Mor10], who has pioneered the stability approach to BSDE with jumps specifically for exponential utility, fit into our framework and demonstrate by concrete examples some notable differences in scope in relation to complementary approaches. To this end, let us consider the same utility problem but now in a financial market with pure-jump asset price processes, possibly of infinite activity (as e.g. in the CGMY model of [CGMY02]), and with constraints on trading strategies. We note that a pure-jump setting appears as a natural setup for our JBSDE results, which admit for generators that are (roughly said) 'quadratic' in the $u$-argument but not in $z$-argument, differently from, e.g., [Mor10, KTPZ15, LS14, AM16, Yao17].

Let $\mu=\mu^{L}$ be the random measure associated to a pure-jump Lévy process $L$ with Lévy measure $\lambda(\mathrm{d} e)$, on $E=\mathbb{R}^{1} \backslash\{0\}$. Let $\mathbb{F}=\mathbb{F}^{L}$ be the usual filtration generated by $L$. The compensated random measure $\widetilde{\mu}=\widetilde{\mu}^{L}:=\mu^{L}-\nu$, with $\nu(\mathrm{d} t, \mathrm{~d} e)=\lambda(\mathrm{d} e) \mathrm{d} t$ of $L$ alone has the weak PRP w.r.t. the filtration $\mathbb{F}$ (see Example 2.1.1.). Note that $\mu$ could be of infinite activity, i.e. $\lambda(E) \leq \infty$, for instance for $L$ being a Gamma process. In contrast to the setup of Section 5.1.1, we consider now a financial market whose single risky asset prices evolves in a non-continuous fashion, being given by a pure-jump process

$$
\mathrm{d} S_{t}=S_{t-}\left(\beta_{t} \mathrm{~d} t+\int_{E} \psi_{t}(e) \widetilde{\mu}(\mathrm{d} t, \mathrm{~d} e) \quad \text { for } t \in[0, T], \text { with } S_{0} \in(0, \infty)\right.
$$

where $\beta$ is predictable and bounded, and $\psi>-1$ is $\widetilde{\mathcal{P}}$-measurable, in $L^{2}(\mathbb{P} \otimes \lambda \otimes \mathrm{d} t) \cap L^{\infty}(\mathbb{P} \otimes \lambda \otimes \mathrm{d} t)$ and satisfies $\int_{E}\left|\psi_{t}(e)\right|^{2} \lambda(\mathrm{d} e) \leq$ const. $\mathbb{P} \otimes \mathrm{d} t$-a.e.. The set $\Theta$ of admissible trading strategies consists of all $\mathbb{R}$-valued predictable $S$-integrable processes $\theta \in L^{2}(\mathbb{P} \otimes \mathrm{d} t)$, such that $\theta_{t}(\omega) \in C$ for all $(t, \omega)$, for a fixed compact set $C \subset \mathbb{R}$ of trading constraint containing 0 . Interpreting trading strategies $\theta$ as amount of wealth invested into the risky asset yields wealth process $X^{\theta, t, x}$ from initial capital $x$ at time $t$ as

$$
X_{s}^{\theta, t, x}=X_{t}^{\theta, t, x}+\int_{t}^{s} \theta_{u} \frac{\mathrm{d} S_{u}}{S_{u-}}=x+\int_{t}^{s} \theta_{u}\left(\beta_{u} \mathrm{~d} u+\int_{E} \psi_{u}(e) \widetilde{\mu}(\mathrm{d} u, \mathrm{~d} e)\right), \quad s \geq t .
$$

Because of the compactness of $C$ and the fact that $\psi \in L^{2}(\mathbb{P} \otimes \lambda \otimes \mathrm{d} t) \cap L^{\infty}(\mathbb{P} \otimes \lambda \otimes \mathrm{d} t)$, admissible strategies are bounded and for all $\theta \in \Theta$ one can verify that $\left\{\exp \left(-\alpha X_{\tau}^{\theta}\right) \mid \tau\right.$ an $\mathbb{F}$-stopping time $\}$ is uniformly integrable; arguments being like in [Mor10, Lem.1]. Consider the JBSDE

$$
-\mathrm{d} Y_{t}=f\left(t, U_{t}\right) \mathrm{d} t-\int_{E} U_{t}(e) \widetilde{\mu}(\mathrm{d} t, \mathrm{~d} e), \quad Y_{T}=\xi,
$$

with terminal condition $\xi \in L^{\infty}\left(\mathcal{F}_{T}\right)$ and generator $f$ defined pointwise by

$$
f(t, u):=\inf _{\theta \in C}\left(-\theta \beta_{t}+\int_{E} g_{\alpha}\left(u(e)-\theta \psi_{t}(e)\right) \lambda(\mathrm{d} e)\right), \quad t \in[0, T]
$$

for the function $g_{\alpha}: \mathbb{R} \rightarrow \mathbb{R}$ with $g_{\alpha}(u):=\left(\mathrm{e}^{\alpha u}-\alpha u-1\right) / \alpha$. We have the following

Proposition 5.2. Let $(Y, U) \in \mathcal{S}^{\infty} \times \mathcal{L}^{2}(\widetilde{\mu})$ be the unique solution to the JBSDE (5.5). Then the strategy $\theta^{*}$ such that $\theta_{t}^{*}$ achieves the infimum in (5.6) for $f\left(t, U_{t}\right)$ is optimal for the control problem (5.3) and achieves at any $t \in[0, T]$ the maximal expected exponential utility $v_{t}(x)=$ $-\exp \left(-\alpha\left(x-Y_{t}\right)\right)=V_{t}^{\theta^{*}}$. 
Proof. Using the martingale optimality principle one obtains, like in the cited literature and analogously to Section 5.1.1, that if $(Y, U) \in \mathcal{S}^{\infty} \times \mathcal{L}^{2}(\widetilde{\mu})$ is a solution to the JBSDE (5.5) then the solution to the utility maximization problem (5.3) is indeed given by $v_{t}(x)=u\left(x-Y_{t}\right)$ (recall that $u$ denotes the exponential utility function) with the strategy $\theta^{*}$ where $\theta_{t}^{*}(\omega)$ achieves the infimum $f\left(\omega, t, U_{t}(\omega)\right)$ in (5.6) for all $(\omega, t)$ being optimal (it exists by measurable selection [Roc76]). To complete the derivation of this example, it thus just remains to show that the JBSDE (5.5) indeed admits a unique solution, with trivial $Z$-component $Z=0$. This is shown by applying Theorem 4.11 and Corollary 4.12 since $\xi \in L^{\infty}\left(\mathcal{F}_{T}^{L}\right)$ and the generator $f$ does not have a $z$-argument and is $\mathbb{F}^{L}$-predictable in $(t, \omega)$. It is straightforward, albeit somewhat tedious, to verify that the conditions 1-5 and $\left(B_{\gamma^{n}}\right), n \in \mathbb{N}$, for Theorem 4.11 are indeed satisfied for the sequence of $\mathbb{F}^{L}$-predictable generators functions

$$
f^{n}(t, u):=\inf _{\theta \in C}\left(-\theta \beta_{t}+\int_{A_{n}} g_{\alpha}\left(u(e)-\theta \psi_{t}(e)\right) \lambda(\mathrm{d} e)\right)
$$

where $\left(A_{n}\right)_{n}$ is a sequence of measurable sets with $A_{n} \uparrow E$ and $\lambda\left(A_{n}\right)<\infty$ for all $n \in \mathbb{N}$, typically $A_{n}=(-\infty,-1 / n] \cup[1 / n,+\infty)$. Let us refer to [Ken15, Example 1.32] for details of this verification, but explain here how to proceed further with the proof.

By the first claim of Theorem 4.11 (together with Corollary 4.12) one then gets existence of a solution $(Y, U) \in \mathcal{S}^{\infty} \times \mathcal{L}^{2}(\widetilde{\mu})$ to the $\operatorname{JBSDE}(5.5)$, such that $U * \widetilde{\mu}$ is a BMO-martingale. To obtain uniqueness by applying the second claim, we need to check that $f$ satisfies condition $\left(\mathbf{A}_{\gamma}^{\prime}\right)$ : To this end, we define $\gamma_{t}^{u, u^{\prime}}(e):=\sup _{\theta \in C} \gamma_{t}^{\theta, u, u^{\prime}}(e) \mathbb{1}_{\left\{u \geq u^{\prime}\right\}}+\inf _{\theta \in C} \gamma_{t}^{\theta, u, u^{\prime}}(e) \mathbb{1}_{\left\{u<u^{\prime}\right\}}$, for $\gamma_{t}^{\theta, u, u^{\prime}}(e):=\int_{0}^{1} g_{\alpha}^{\prime}\left(l\left(u-\theta \psi_{t}(e)\right)+(1-l)\left(u^{\prime}-\theta \psi_{t}(e)\right)\right) \mathrm{d} l$. Then (by Examples 3.5 and 3.8-2.) we get $f\left(t, U_{t}\right)-f\left(t, U_{t}^{\prime}\right) \leq \int_{E} \gamma_{t}^{U, U^{\prime}}(e)\left(U_{t}(e)-U_{t}^{\prime}(e)\right) \lambda(\mathrm{d} e)$ for all $U, U^{\prime}$ with $|U|_{\infty}<\infty,\left|U^{\prime}\right|_{\infty}<\infty$. Now let $u, u^{\prime}$ be bounded by $c>0$; Since $g_{\alpha}^{\prime}(0)=0$, applying the mean-value theorem to $g_{\alpha}^{\prime}$ in the expression of $\gamma^{\theta, u, u^{\prime}}$ gives $\left|\gamma_{t}^{\theta, u, u^{\prime}}(e)\right| \leq \sup _{|x| \leq \tilde{c}}\left|g_{\alpha}^{\prime \prime}(x)\right|\left(|u|+\left|u^{\prime}\right|+|\theta|\left|\psi_{t}(e)\right|\right)$ for all $\theta \in C$, where $\tilde{c}:=c+\|\psi\|_{\infty} \operatorname{diam}(C)$. This implies (for $c=|U|_{\infty} \vee\left|U^{\prime}\right|_{\infty}<\infty$ )

$$
\sup _{\theta \in C}\left|\gamma_{t}^{\theta, U, U^{\prime}}(e)\right| \leq \sup _{|x| \leq \tilde{c}}\left|g_{\alpha}^{\prime \prime}(x)\right|\left(\left|U_{t}(e)\right|+\left|U_{t}^{\prime}(e)\right|+\operatorname{diam}(C)\left|\psi_{t}(e)\right|\right) \text {. }
$$

Since $\left|\inf _{\theta} \gamma^{\theta}\right| \leq \sup _{\theta}\left|\gamma^{\theta}\right|,\left|\sup _{\theta} \gamma^{\theta}\right| \leq \sup _{\theta}\left|\gamma^{\theta}\right|$ and $\psi * \widetilde{\mu}$ is a BMO-martingale (as $\psi$ is bounded and $\int_{E}\left|\psi_{t}(e)\right|^{2} \lambda(\mathrm{d} e) \leq$ const., $\mathbb{P} \otimes \mathrm{d}$ t-a.e. by assumption), then $\gamma^{U, U^{\prime}} * \widetilde{\mu}$ is a BMO-martingale if $U * \widetilde{\mu}$ and $U^{\prime} * \widetilde{\mu}$ are, thanks to $|U|_{\infty}<\infty$ and $\left|U^{\prime}\right|_{\infty}<\infty$. Hence $f$ satisfies $\left(\mathbf{A}_{\gamma}^{\prime}\right)$.

Example 5.3. (entropic convex risk measure) Let us consider the special case $\beta=\psi \equiv 0$ and $S \equiv 1$, i.e. the exponential utility problem without trading opportunities in a risky asset. One gets the important and well known example of the (dynamic) entropic risk measure $Y_{t}=(1 / \alpha) \log \mathbb{E}\left(\exp (\alpha \xi) \mid \mathcal{F}_{t}\right)$ whose JBSDE description can be identified directly by exponential transformation. In the setup of the present subsection, this JBSDE is covered by the application study of [Mor10] and also by our comparison and wellposedness theorems, without any further conditions on the pure-jump Lévy process. In contrast, let us demonstrate that the same scope is not already offered by the seminal comparison Thm.2.5 of [Roy06] because her key condition $\left(\mathbf{A}_{\boldsymbol{\gamma}}\right)$ is not satisfied, which is also supposed for results in [KTPZ15, as Assumpt.6.1 for wellposedness in Thm.6.3(i) and for comparison in Prop.6.4] (and is further used for applications in [KTPZ16]): Indeed for $\alpha:=1$, just consider a compound Poisson process $L$ (being of finite activity) with uniformly distributed jump heights, taking $\lambda(\mathrm{d} x):=\mathbb{1}_{(0,1]} \mathrm{d} x$ $(x \in E=\mathbb{R} \backslash\{0\})$. Clearly, the generator $f(u)=\int_{E} \exp (u(x))-u(x)-1 \lambda(\mathrm{d} x)=: \int_{E} g(u(x)) \lambda(\mathrm{d} x)$ is not Lipschitz in $u \in L^{2}(\lambda)$. With $u^{ \pm}(x):=\frac{1}{2}\left( \pm x^{-3 / 2}+n x\right) \mathbb{1}_{(1 / n, 1]}(x)$ in $L^{2}(\lambda)$ for $n \in \mathbb{N}$, we get $\int_{0}^{1}\left(\mathrm{e}^{u^{+}}-\mathrm{e}^{u^{-}}-u^{+}+u^{-}\right) \mathrm{d} \lambda \rightarrow \infty$ for $n \rightarrow \infty$ while $\int_{0}^{1}\left(u^{+}-u^{-}\right)(x) \cdot(1 \wedge|x|) \mathrm{d} x \leq \int_{0}^{1} x^{-1 / 2} \mathrm{~d} x<\infty$ for all $n$, noting that $\mathrm{e}^{u^{+}}-\mathrm{e}^{u^{-}}-u^{+}+u^{-} \geq n x^{-1 / 2} \mathbb{1}_{(1 / n, 1]}$. Thus, there cannot be constants $c_{1} \in(-1,0], c_{2}<\infty$, such that $f(u)-f(v) \leq \int_{E}(u-v)(x) \gamma^{u, v}(x) \lambda(\mathrm{d} x)$ for all $u, v$, with $c_{1}(1 \wedge|x|) \leq \gamma^{u, v}(x) \leq c_{2}(1 \wedge|x|)$; This shows that condition $\left(\mathbf{A}_{\boldsymbol{\gamma}}\right)$ in [Roy06] or Assumption 6.1 in [KTPZ15], are not satisfied here. Indeed, the seminal $\left(\mathbf{A}_{\gamma}\right)$ condition from [Roy06] implies Lipschitz continuity of the generator in $u$.

Similarly, a related condition on the jump measure has been stated in the assumptions of the main theorem in [AM16, Thm. 1, see their inequality (4)]; Their article further assumes 
(like also [Yao17], for instance) finite jump activity in that $\lambda(E)<\infty$ holds (in our notation, they write $\eta$ for $\lambda$ ), what is true in the present example (but is not required for [Roy06], or our previous sections). But in this example there cannot exist constants $c_{1} \in(-1,0], c_{2}<\infty$ such that $f(u)-f\left(u^{\prime}\right) \leq \int_{E}\left(u-u^{\prime}\right)(x) \gamma^{u, u^{\prime}}(x) \lambda(\mathrm{d} x)$ would hold for all $u, u^{\prime} \in L^{2}(\lambda)$, with suitable functions $c_{1} \leq \gamma^{u, u^{\prime}}(x) \leq c_{2}$. Indeed, the latter would imply (using Cauchy-Schwarz inequality) that $f$ is Lipschitz in $u$ on $L^{2}(\lambda)$, what is not true. Hence, the assumptions for Theorem 1 in [AM16] appear not satisfied for the entropic risk example, and its conditions (with inequality (4) assumed for all $\left.u, u^{\prime}\right)$ would imply that the JBSDE generator has to be Lipschitz continuous in $u \in L^{2}(\lambda)$. Note that the aforementioned problem could be resolved if, e.g., an additional $L^{\infty}$-bound is available for the $u$-argument, for instance by an exogenuous a-priori $L^{\infty}$-estimate on the $U$-component for bounded JBSDE solutions (like from Section 3). We note that [AM16] offer results for unbounded JBSDE solutions (see also [HLT17] for exponential utility in the continuous case without jumps).

Example 5.4. We continue with the previous entropic risk example, but now take a standard Poisson process instead, i.e. $\lambda(\mathrm{d} e)=\delta_{\{1\}}(\mathrm{d} e)$ as Dirac point measure at the fixed jump height 1. Then $L^{2}(\lambda)$ is isomorphic to $\mathbb{R}$, and $f(u)=g(u)=\exp (u)-u-1$ for $u \in \mathbb{R}$. Obviously $g^{\prime}$ and $g^{\prime \prime}$ are of exponential growth (in $u$ ) and cannot be bounded globally in $u \in \mathbb{R}$ by an affine function or by constants; hence Assumption 5.1(iii) for [KTPZ15, Thm.5.4] cannot be satisfied. Moreover, also Assumption 4.3(iii) for [KTPZ15, Thms.4.3, 5.4 and 6.3(ii)], noting their Lem.5.4, appears clearly violated since (taking $u^{\prime}=0$ ) there exist no $\psi, c \in \mathbb{R}$ such that $|g(u)-\psi u| \leq c|u|^{2}$ for all $u \in \mathbb{R}$.

Note that, since the function $(u, \theta) \mapsto f^{\theta}(\cdot, u):=-\theta \beta .+\int_{E} g_{\alpha}(u(e)-\theta \psi \cdot(e)) \lambda(\mathrm{d} e)$ is convex, the generator constructed as $f=\inf _{\theta \in C} f^{\theta}(\cdot, u)$ (cf. 5.6) would be convex in $u$ if the constraint set $C$ were assumed to be convex; But for non-convex trading constraints $C$ the generator can be non-convex in general. Similar constructions of generators are typical in this application context, see e.g. [LS14, equation (15)]. Some results on JBSDE in the literature use convexity of the generator function but that can be restrictive for applications. For results in the present paper, convexity is not being assumed. Next, we give a concrete application example where $f$ in (5.6) for the (primal) control problem is indeed non-convex in $u$. The example shows, how non-convex constraints can lead to JBSDE generators which are non-convex in $u$. To this end, let us consider simple trading constraints that are non-convex by taking $C:=\left\{\theta^{0}, \ldots, \theta^{m}\right\} \subset \mathbb{R}$ as a finite set including the zero $\theta^{0}:=0$. Here $f$ of JBSDE (5.5) becomes

$$
f(t, u)=\inf _{k \in\{0, \ldots, m\}}\left(-\theta^{k} \beta_{t}+\int_{E} g_{\alpha}\left(u(e)-\theta^{k} \psi_{t}(e)\right) \lambda(\mathrm{d} e)\right) .
$$

Example 5.5. (An application where the generator is not convex and not continuously differentiable) Continuing with the above generator $f$, now let us take the particular case where $\lambda(\mathrm{d} e)=\delta_{\{1\}}(\mathrm{d} e)$, i.e. $L$ is a standard Poisson process with constant jump height 1 , and let $\alpha=1$ and $\beta=0$. Observing that $L^{2}(\lambda)$ in the case of this simple example is isomorphic to $\mathbb{R}$, we see that

$$
f(t, u)=\min _{k \in\{0, \ldots, m\}}\left(\mathrm{e}^{\left(u-\theta^{k} \psi_{t}\right)}-\left(u-\theta^{k} \psi_{t}\right)-1\right)
$$

is clearly non-convex in $u \in \mathbb{R}$, unless $\psi \equiv 0$ or $C=\{0\}$. Also, we observe that $u \mapsto f(t, u)$ is not continuously differentiable in $u \in \mathbb{R}$ for this application. But the function $f$ in (5.7) is still absolutely continuous in $u$ with density function being strictly greater than -1 , locally bounded in $u$ from above and locally of linear growth. Because this $f$ satisfies condition $\left(\mathbf{A}_{\mathbf{i n i}}\right)$, existence and uniqueness for the corresponding JBSDE (5.5) solution can be obtained by Theorem 4.13.

Similarly as before, one can check that in this example the assumptions of [Roy06, Thm.2.5] and [KTPZ15, Thm.5.4, Thm.6.3(i)-(ii), Prop.6.4] for comparison and wellposedness of JBSDE are not satisfied; The example clearly shows how non-convex constraints can indeed lead to a non-convex $f$, which does not satisfy the conditions for the JBSDE results of [LS14, Thm.A28, Cor.A29, Prop.A30, being used further in the proofs for Thms.4.3, 4.5, all involving convexity assumption "(c)"].

We proceed next with examples beyond exponential utility, that was the topic in [Mor09, Mor10]. 


\subsection{Power utility maximization}

Again for the market with stock price dynamics (5.1), we consider the utility maximization problem

$$
v_{t}(x)=\underset{\theta \in \Theta}{\operatorname{ess} \sup _{\theta}} \mathbb{E}\left(u\left(X_{T}^{\theta, t, x}\right) \xi \mid \mathcal{F}_{t}\right)=\frac{1}{\gamma} \operatorname{ess}_{\theta \in \Theta} \sup _{\theta} \mathbb{E}\left(u\left(X_{T}^{\theta, t, x} \xi^{\prime}\right) \mid \mathcal{F}_{t}\right), \quad t \leq T, x>0,
$$

for power utility $u(x)=x^{\gamma} / \gamma$ with relative risk aversion $1-\gamma>0$ for $\gamma \in(0,1)$, with multiplicative liability $\xi$ (alternatively, $\xi^{\prime}:=(\gamma \xi)^{1 / \gamma}$ can be interpreted as an unknown future tax rate). The wealth process of strategy $\theta$ (denoting fraction of wealth invested) is $X_{s}^{\theta}=X_{s}^{\theta, t, x}=x+\int_{t}^{s} X_{u}^{\theta} \theta_{u} \mathrm{~d} \widehat{B}_{u}=$ $x \mathcal{E}\left(\int \theta \mathrm{d} \widehat{B}\right)_{t}^{s}$ for $s \in[t, T]$, for $\theta \in \Theta$, with the set $\Theta$ of strategies given by all $\mathbb{R}^{d}$-valued, predictable, $S$-integrable processes such that $\theta \bullet B$ is a $\operatorname{BMO}(\mathbb{P})$-martingale, cf. [HWY92].

Proposition 5.6. Let $k=d$. Assume that there is a solution $(Y, Z, U) \in \mathcal{S}^{\infty} \times \mathcal{L}^{2}(B) \times \mathcal{L}^{2}(\widetilde{\mu})$ to the $\operatorname{BSDE}(\xi, f)$ with $f_{t}(y, z, u):=(\gamma /(2-2 \gamma)) y\left|\varphi_{t}+y / z\right|^{2}$ and $\int Z \mathrm{~d} B \in \mathrm{BMO}(\mathbb{P})$ and where $\xi$ is in $L^{\infty}\left(\mathcal{F}_{T}\right)$ with $\xi \geq c$ for some $c>0$. Then $Y \geq c$ holds and $V^{\theta}:=u\left(X^{\theta}\right) Y$ is a supermartingale for all $\theta$ in $\Theta$ and $V^{\theta^{*}}$ is a martingale for $\theta^{*}:=(1-\gamma)^{-1}\left(\varphi+Z / Y_{-}\right) \in \Theta$.

Proof. Clearly, $V^{\theta}$ is adapted. Kazamaki's criterion $\mathcal{E}\left(\int_{0}^{\cdot} \gamma \theta_{u} \mathrm{~d} \widehat{B}_{u}\right)$ is an $r$-integrable martingale for some $r>1$. Hence $\sup _{t \leq s \leq T} \mathcal{E}\left(\int_{0}^{\cdot} \gamma \theta_{u} \mathrm{~d} \widehat{B}_{u}\right)_{t}^{s}$ is integrable by Doob's inequality. By

$$
\mathcal{E}(\theta \cdot \widehat{B})^{\gamma}=\mathcal{E}(\gamma \theta \cdot \widehat{B}) \exp \left(-\frac{1}{2} \gamma(1-\gamma) \int_{0}\left|\theta_{u}\right|^{2} \mathrm{~d} u\right) \leq \mathcal{E}(\gamma \theta \cdot \widehat{B}),
$$

we conclude that $V^{\theta}$ is dominated by $\sup _{t \leq s \leq T} U\left(X_{s}^{\theta}\right)|Y|_{\infty} \in L^{1}(\mathbb{P})$. By Itô's formula, $\mathrm{d} V_{s}^{\theta}$ equals a local martingale plus the finite variation part

$$
u\left(X_{s}^{\theta}\right)\left(-f_{s}\left(Y_{s-}, Z_{s}, U_{s}\right)+\gamma\left(Y_{s-}\left(\theta_{s} \varphi_{s}+\frac{1}{2}(\gamma-1)\left|\theta_{s}\right|^{2}\right)+\theta_{s} Z_{s}\right)\right) \mathrm{d} s .
$$

The latter part is decreasing for all $\theta \in \Theta$ and vanishes at zero for $\theta=\theta^{*}$. So $V^{\theta}$ is a local (super)martingale. Uniform integrability of $V^{\theta}$ yields the (super)martingale property. By the classical martingale optimality principle of optimal control follows that $v_{t}(x)=\operatorname{ess}_{\sup _{\theta \in \Theta}} \mathbb{E}\left(u\left(X_{T}^{\theta} \xi^{1 / \gamma}\right) \mid \mathcal{F}_{t}\right)$ equals $V_{t}^{\theta^{*}}=\gamma^{-1} x^{\gamma} Y_{t}$, and evaluating at $\theta \equiv 0$ yields $\gamma^{-1} x^{\gamma} \mathbb{E}\left(\xi \mid \mathcal{F}_{t}\right) \leq \gamma^{-1} x^{\gamma} Y_{t}$ and hence $Y \geq c$. Note that $\theta^{*}$ is in $\Theta$ since $\varphi$ is bounded, $Y$ is bounded away from 0 and $Z$ is an BMO integrand.

Let $(Y, Z, U)$ be a solution to the $\operatorname{BSDE}(\xi, f)$ with the above data. Since a suitable solution theory for quadratic BSDEs with jumps is not available, we transform coordinates by letting

$$
\widetilde{Y}_{t}:=Y_{t}^{\frac{1}{1-\gamma}}, \quad \widetilde{Z}_{t}:=\frac{1}{1-\gamma} Y_{t-}^{\frac{\gamma}{1-\gamma}} Z_{t} \quad \text { and } \quad \widetilde{U}_{t}:=\left(Y_{t-}+U_{t}\right)^{\frac{1}{1-\gamma}}-Y_{t-}^{\frac{1}{1-\gamma}}
$$

such that $(\tilde{Y}, \widetilde{Z}, \widetilde{U})$ solves the BSDE for data $(\widetilde{\xi}, \widetilde{f})$ with $\widetilde{\xi}=\xi^{1 /(1-\gamma)}$ and $\widetilde{f}_{t}(y, z, u)$ given by

$$
\frac{\gamma\left|\varphi_{t}\right|^{2}}{2(1-\gamma)^{2}} y+\frac{\gamma}{1-\gamma} \varphi_{t} z+\int_{E}\left(\frac{1}{1-\gamma}\left((u(e)+y)^{1-\gamma} y^{\gamma}-y\right)-u(e)\right) \zeta(t, e) \lambda(\mathrm{d} e) .
$$

Looking at the proof of Lemma 2.2, we may assume that $U+Y_{-}$coincides pointwise with $Y_{-}$or $Y$ so that the above transformation is well-defined due to $Y \geq c$. In fact, (5.9) gives a bijection between solutions with positive Y-components to the $\operatorname{BSDEs}(\xi, f)$ and $(\widetilde{\xi}, \widetilde{f})$ in $\mathcal{S}^{\infty} \times \mathcal{L}^{2}(B) \times \mathcal{L}^{2}(\widetilde{\mu})$.

Next, we show the existence of a JBSDE solution for data $(\xi, f)$ with $\xi \geq c$ for some $c>0$. Under the probability measure $\mathrm{d} \widetilde{\mathbb{P}}:=\mathcal{E}\left(\gamma(1-\gamma)^{-1} \varphi \cdot B\right)_{T} \mathrm{~d} \mathbb{P}$ the process $\widetilde{B}=B-\int_{0} \gamma(1-\gamma)^{-1} \varphi_{t} \mathrm{~d} t$ is a Brownian motion and the JBSDE

$$
\widetilde{Y}_{t}=\widetilde{\xi}+\int_{t}^{T} \widetilde{f}_{s}\left(\widetilde{Y}_{s-}, \widetilde{Z}_{s}, \widetilde{U}_{s}\right) \mathrm{d} s-\int_{t}^{T} \widetilde{Z}_{s} \mathrm{~d} B_{s}-\int_{t}^{T} \int_{E} \widetilde{U}_{s}(e) \widetilde{\mu}(\mathrm{d} s, \mathrm{~d} e)
$$

under $\mathbb{P}$ is of the following form under $\widetilde{\mathbb{P}}$

$$
\tilde{Y}_{t}=\widetilde{\xi}+\int_{t}^{T}\left(\widetilde{f}_{s}\left(\widetilde{Y}_{s-}, \widetilde{Z}_{s}, \widetilde{U}_{s}\right)-\frac{\gamma \varphi_{s}}{1-\gamma} \widetilde{Z}_{s}\right) \mathrm{d} s-\int_{t}^{T} \widetilde{Z}_{s} \mathrm{~d} \widetilde{B}_{s}-\int_{t}^{T} \int_{E} \widetilde{U}_{s}(e) \widetilde{\mu}(\mathrm{d} s, \mathrm{~d} e),
$$

noting that $\nu$ is the compensator of $\mu$ under $\mathbb{P}$ and $\widetilde{\mathbb{P}}$ as well. In fact, we have 
Lemma 5.7. Assume $\lambda(E)<\infty$. Then $(\widetilde{Y}, \widetilde{Z}, \widetilde{U}) \in \mathcal{S}^{\infty} \times \mathcal{L}^{2}(B) \times \mathcal{L}^{2}(\widetilde{\mu})$ solves the BSDE $(\widetilde{\xi}, \widetilde{f})$ such that $\int \widetilde{Z} \mathrm{~d} B$ is in $\mathrm{BMO}(\mathbb{P})$ if and only if $(\widetilde{Y}, \widetilde{Z}, \widetilde{U}) \in \mathcal{S}_{\widetilde{\mathbb{P}}}^{\infty} \times \mathcal{L}_{\widetilde{\mathbb{P}}}^{2}(\widetilde{B}) \times \mathcal{L}_{\widetilde{\mathbb{P}}}^{2}(\widetilde{\mu})$ solves the BSDE $\left(\widetilde{\xi}, \widetilde{f}(y, z, u)-\gamma(1-\gamma)^{-1} \varphi z\right)$ such that $\int \widetilde{Z} \mathrm{~d} \widetilde{B}$ is in $\operatorname{BMO}(\widetilde{\mathbb{P}})$.

Proof. Equivalence of $\mathbb{P}$ and $\widetilde{\mathbb{P}}$ imply that $\widetilde{Y} \in \mathcal{S}^{\infty}$ if and only if $\widetilde{Y} \in \mathcal{S}_{\widetilde{\mathbb{P}}}^{\infty}$. Given $\lambda(E)<\infty$, $\widetilde{U} \in \mathcal{L}^{2}(\widetilde{\mu})$ holds if and only if $\widetilde{U} \in \mathcal{L}_{\widetilde{\mathbb{P}}}^{2}(\widetilde{\mu})$ due to the boundedness of $\widetilde{U}$. By [Kaz94, Thm.3.6], the restriction of the Girsanov transform $\Phi: \mathcal{M}_{\mathrm{c}}^{\text {loc }, 0}(\mathbb{P}) \longrightarrow \mathcal{M}_{\mathrm{c}}^{\text {loc }, 0}(\widetilde{\mathbb{P}})$, with $M \mapsto M-\left\langle M, \int_{0} \frac{\gamma \varphi}{1-\gamma} \mathrm{d} B_{s}\right\rangle$, onto $\mathrm{BMO}(\mathbb{P})$ yields a bijection between $\mathrm{BMO}(\mathbb{P})$-martingales and $\mathrm{BMO}(\widetilde{\mathbb{P}})$-martingales. Thus, $\int \widetilde{Z} \mathrm{~d} B$ is in $\operatorname{BMO}(\mathbb{P})$ if and only if $\int \widetilde{Z} \mathrm{~d} \widetilde{B}$ is in $\operatorname{BMO}(\widetilde{\mathbb{P}})$ for $Z=(1-\gamma) \widetilde{Y}_{-}^{\gamma} \widetilde{Z}$ since $\Phi\left(\int \widetilde{Z} \mathrm{~d} B\right)=$ $\int \widetilde{Z} \mathrm{~d} B-\int \gamma(1-\gamma)^{-1} \varphi \widetilde{Z}_{s} \mathrm{~d} s=\int \widetilde{Z} \mathrm{~d} \widetilde{B}$. In particular, $\widetilde{Z} \in \mathcal{L}^{2}(B)$ iff $\widetilde{Z} \in \mathcal{L}_{\widetilde{\mathbb{P}}}^{2}(\widetilde{B})$.

To proceed further, let us note at first that under an equivalent change of measure between $\mathbb{P}$ and $\mathbb{P}$, the weak predictable representations property for $(B, \widetilde{\mu})$ under $\mathbb{P}$ is equivalent to the respective property of $(\widetilde{B}, \widetilde{\mu})$ under $\widetilde{\mathbb{P}}$ for the same filtration, see [HWY92, Theorem 13.22] and recall Example 2.1, Part 2. According to Corollary 4.6, hence there exists a unique solution $(\widetilde{Y}, \widetilde{Z}, \widetilde{U}) \in \mathcal{S}_{\widetilde{\mathbb{P}}}^{\infty} \times \mathcal{L}_{\mathbb{\mathbb { P }}}^{2}(B) \times \mathcal{L}_{\widetilde{\mathbb{P}}}^{2}(\widetilde{\mu})$ with positive $Y$-component to the $\operatorname{BSDE}(5.10)$ with

$$
c^{\frac{1}{1-\gamma}} \exp \left(-\frac{\gamma|\varphi|_{\infty}^{2}}{2(1-\gamma)^{2}}(T-t)\right) \leq \widetilde{Y}_{t} \leq|\xi|_{\infty} \exp \left(\frac{\gamma|\varphi|_{\infty}^{2}}{2(1-\gamma)^{2}}(T-t)\right)
$$

such that $\int \widetilde{Z} \mathrm{~d} \widetilde{B}$ and $\widetilde{U} * \widetilde{\mu}^{\mathbb{P}}$ are $\operatorname{BMO}(\widetilde{\mathbb{P}})$-martingales. By Lemma 5.7 and the statement of Proposition 5.6 that every bounded solution to the $\operatorname{BSDE}(\xi, f)$ is bounded from below away from zero in $Y \geq c>0$, there is a unique solution $(Y, Z, U)$ in $\mathcal{S}^{\infty} \times \mathcal{L}^{2}(B) \times \mathcal{L}^{2}(\widetilde{\mu})$ with $\int Z \mathrm{~d} B \in \operatorname{BMO}(\mathbb{P})$ and it is given by the coordinate transform (5.9). We note that $Y$ (resp. $\widetilde{Y}$ ) can be interpreted as (dual) opportunity process, see [Nut10, Sect.4]. Overall, we obtain the next theorem.

Theorem 5.8. Assume $\lambda(E)<\infty$ and $d=k$. Let $f_{s}(y, z, u)=\gamma(2-2 \gamma)^{-1} y\left|\varphi_{s}+z / y\right|^{2}$ and let $\xi \in L^{\infty}\left(\mathcal{F}_{T}\right)$ with $\xi \geq c$ for some $c>0$. Then there exists a unique solution $(Y, Z, U) \in \mathcal{S}^{\infty} \times \mathcal{L}^{2}(B) \times$ $\mathcal{L}^{2}(\widetilde{\mu})$ with $\int Z \mathrm{~d} B \in \operatorname{BMO}(\mathbb{P})$ to the $B S D E(\xi, f)$. Then the strategy $\theta_{s}^{*}=(1-\gamma)^{-1}\left(\varphi_{s}+Z_{s} / Y_{s-}\right)$ is optimal for the control problem (5.8), achieving $v_{t}(x)=\gamma^{-1} x^{\gamma} Y_{t}=V_{t}^{\theta^{*}}$.

\subsection{Valuation by good-deal bounds}

In incomplete financial markets without arbitrage, there exist infinitely many pricing measures and the bounds imposed on valuation solely by the principle of no-arbitrage are typically far too wide for applications in practice. Good-deal bounds [CSR00] have been introduced in the finance literature to obtain tighter bounds, by ruling out not only arbitrage but also trading opportunities with overly attractive reward-for-risk ratios, so-called good deals. See [BK17b, BK17a] for extensive references and applications under model ambiguity. In [CSR00, BS06] good deals have been defined in terms of too favorable instantaneous Sharpe ratios (rate of excess return per unit rate of volatility) for continuous diffusion processes. This has been generalized to a jump-diffusion setup by [BS06], who describe good-deal bounds as solutions of nonlinear partial-integro differential equations by using (formal) HJB methods. We complement their work here by a rigorous, possibly non-Markovian, description by JBSDEs. See [DP15] for a study of a case where the measure $\lambda$ has finite support.

In our setting, the following description of the set $\mathcal{M}^{\mathrm{e}}$ of martingale measures is routine.

Proposition 5.9. $\mathcal{M}^{\mathrm{e}}$ consists of those measures $\mathbb{Q} \approx \mathbb{P}$ such that $\mathrm{d} \mathbb{Q} / \mathrm{d} \mathbb{P}=\mathcal{E}(\beta \bullet B+\gamma * \widetilde{\mu})$, where $\gamma>-1$ is a $\widetilde{\mathcal{P}}$-measurable and $\widetilde{\mu}$-integral function, and $\beta$ is a predictable process with $\int_{0}^{T}\left|\beta_{s}\right|^{2} \mathrm{~d} s<\infty$, satisfying $\beta=-\varphi+\eta$, such that $\eta \in \operatorname{Ker} \sigma, \mathbb{P} \otimes \mathrm{d} t$-a.e..

We will refer to the tuple $(\gamma, \beta)$ for such a density $d \mathbb{Q} / d \mathbb{P}$ as the Girsanov kernel of $\mathbb{Q}$ relative to $\mathbb{P}$. Clearly, our market is incomplete in general as there exists infinitely many measures in $\mathcal{M}^{\mathrm{e}}$ if $\widetilde{\mu}$ is non-trivial or $k<d$. Björk and Slinko employed an extended Hansen-Jagannathan inequality [BS06, see Sect.2] to bound the instantaneous Sharpe ratio by imposing a bound on market prices 
of risk. More precisely, Thm.2.3 of [BS06] showed that the instantaneous Sharpe ratio $S R_{t}$ in any extension of the market by additional derivative assets (i.e. by any local $\mathbb{Q}$-martingales) satisfies $\left|S R_{t}\right| \leq\left\|\left(\gamma_{t}, \beta_{t}\right)\right\|_{L^{2}\left(\lambda_{t}\right) \times \mathbb{R}^{d}}$ at any time $t$, with a (sharp) upper bound in terms of an $L^{2}$-norm for Girsanov kernels $(\gamma, \beta)$ of pricing measures in $\mathcal{M}^{\mathrm{e}}$, with $\lambda_{t}(\omega)(\mathrm{d} e):=\zeta_{t}(\omega, e) \lambda(\mathrm{d} e)$. As no-good-deal restriction they therefore impose a bound on the kernels of pricing measures

$$
\left\|\left(\gamma_{t}, \beta_{t}\right)\right\|_{L^{2}\left(\lambda_{t}\right) \times \mathbb{R}^{d}}^{2}=\left\|\gamma_{t}\right\|_{L^{2}\left(\lambda_{t}\right)}^{2}+\left|\beta_{t}\right|_{\mathbb{R}^{d}}^{2} \leq K^{2}, \quad t \leq T
$$

by some given constant $K>0$. To complement the analysis of the problem posed by [BS06], we are going to describe the dynamic good deal bounds rigorously by JBSDEs in a more general, possibly non-Markovian, setting with no-good-deal restriction like in (5.11) but, more generally, we allow $K=\left(K_{t}\right)$ to be a positive predictable bounded process instead of a constant.

To this end, for $K$ as above, let the correspondence (set-valued) process $C$ be given by

$$
C_{t}:=\left\{(\gamma, \eta) \in L^{2}\left(\lambda_{t}\right) \times \mathbb{R}^{d} \mid \gamma>-1, \eta \in \operatorname{Ker} \sigma_{t}, \text { and }\|\gamma\|_{L^{2}\left(\lambda_{t}\right)}^{2}+|\eta|_{\mathbb{R}^{d}}^{2}+\left|\varphi_{t}\right|_{\mathbb{R}^{d}}^{2} \leq K_{t}^{2}\right\} .
$$

We will write $(\gamma, \eta) \in C$ to denote that $\eta$ is a predictable process and $\gamma$ is a $\widetilde{\mathcal{P}}$-measurable process with $\left(\gamma_{t}(\omega), \eta_{t}(\omega)\right) \in C_{t}(\omega)$ holding for all $(t, \omega) \in[0, T] \times \Omega$. For $(\gamma, \eta) \in C$, we know (cf. Example 3.3.1) that $\mathcal{E}((-\varphi+\eta) \cdot B+\gamma * \widetilde{\mu})>0$ is a martingale that defines a density process of a probability measure $\mathbb{Q}^{\gamma, \eta}$ which is equivalent to $\mathbb{P}$. The set of such probability measures

$$
\mathcal{Q}^{\text {ngd }}:=\left\{\mathbb{Q}^{\gamma, \eta} \mid(\gamma, \eta) \in C\right\} \subseteq \mathcal{M}^{\mathrm{e}},
$$

defines our set of no-good-deal measures. Beyond boundedness of $\varphi$, assume that $\left|\varphi_{t}\right|_{\mathbb{R}^{d}}+\epsilon<K_{t}$ holds for for some $\epsilon>0$ for all $t \leq T$. Then, in particular, the minimal martingale measure $\widehat{\mathbb{P}}=\mathbb{Q}^{\widehat{\gamma}, \widehat{\eta}}$ is in $\mathcal{Q}^{\text {ngd }} \neq \emptyset$, with $(\widehat{\gamma}, \widehat{\eta}) \equiv(0,0) \in C$. For contingent claims $X \in L^{\infty}(\mathbb{P})$, the processes

$$
\pi_{t}^{u}(X):=\underset{\mathbb{Q} \in \mathcal{Q}^{\text {ngd }}}{\operatorname{ess} \sup _{\mathbb{Q}}} \mathbb{E}_{\mathbb{Q}}\left(X \mid \mathcal{F}_{t}\right) \quad \text { and } \quad \pi_{t}^{l}(X):=\underset{\mathbb{Q} \in \mathcal{Q}^{\text {ngd }}}{\operatorname{ess} \inf } \mathbb{E}_{\mathbb{Q}}\left(X \mid \mathcal{F}_{t}\right), \quad t \leq T,
$$

define the upper and lower good-deal bounds. Noting $\pi^{l}(X)=-\pi^{u}(-X)$, we focus on $\pi^{u}(-X)$. One can check that the good-deal bound process satisfies good dynamic properties, e.g. time-consistency and recursiveness (cf. e.g. [BK17b, Lem.1]). By applying the comparison result of Proposition 3.1, we are going to obtain $\pi^{u}(X)$ as the value process $Y$ of a BSDE with terminal condition $X \in L^{\infty}(\mathbb{P})$. Denoting by $\Pi_{t}(\cdot)$ and $\Pi_{t}^{\perp}(\cdot)$ the orthogonal projections on $\operatorname{Im} \sigma_{t}^{T}$ and Ker $\sigma_{t}$, we have the following lemma (see [Ken15, Lemmas 2.14, 2.22] for details).

Lemma 5.10. For $Z \in \mathcal{L}^{2}(B)$ and $U \in \mathcal{L}^{2}(\widetilde{\mu})$ there exists $\bar{\eta}=\bar{\eta}(Z, U)$ predictable and $\bar{\gamma}=\bar{\gamma}(Z, U)$ $\widetilde{\mathcal{P}}$-measurable such that for $\mathbb{P} \otimes \mathrm{d} t$-almost all $(\omega, t) \in \Omega \times[0, T]$ holds

$$
\bar{\eta}_{t} \Pi_{t}^{\perp}\left(Z_{t}\right)+\int_{E} U_{t}(e) \bar{\gamma}_{t}(e) \zeta_{t}(e) \lambda(\mathrm{d} e)=\max _{(\gamma, \eta) \in \bar{C}}\left(\eta_{t} \Pi_{t}^{\perp}\left(Z_{t}\right)+\int_{E} U_{t}(e) \gamma_{t}(e) \zeta_{t}(e) \lambda(\mathrm{d} e)\right)
$$

where $\bar{C}_{t}=\left\{(\gamma, \eta) \in L^{2}\left(\lambda_{t}\right) \times\left.\mathbb{R}^{d}\left|\gamma \geq-1, \eta \in \operatorname{Ker} \sigma_{t},\|\gamma\|_{L^{2}\left(\lambda_{t}\right)}^{2}+\right| \eta\right|_{\mathbb{R}^{d}} ^{2} \leq K_{t}^{2}-\left|\varphi_{t}\right|_{\mathbb{R}^{d}}^{2}\right\}$ is the closure of $C_{t}$ in $L^{2}\left(\lambda_{t}\right) \times \mathbb{R}^{d}$ for any $t \leq T$.

To $(\bar{\gamma}, \bar{\eta}) \in \bar{C}$ of Lemma 5.10 , we associate a probability measure $\overline{\mathbb{Q}} \ll \mathbb{P}$ defined via $\mathrm{d} \overline{\mathbb{Q}}=$ $\mathcal{E}((-\varphi+\bar{\eta}) \cdot B+\bar{\gamma} * \widetilde{\mu}) \mathrm{d} \mathbb{P}$, which may not be equivalent to $\mathbb{P}$ as $\bar{\gamma}$ may be -1 on a non-negligible set. While $\overline{\mathbb{Q}}$ might not be in $\mathcal{Q}^{\text {ngd }}$ it belongs to the $L^{1}(\mathbb{P})$-closure of $\mathcal{Q}^{\text {ngd }}$ in general, as shown in

Lemma 5.11. For $Z \in \mathcal{L}^{2}(B)$ and $U \in \mathcal{L}^{2}(\widetilde{\mu})$, let $(\bar{\gamma}, \bar{\eta})$ be as in Lemma 5.10. Define the measures $\overline{\mathbb{Q}} \ll \mathbb{P}$ via $\mathrm{d} \overline{\mathbb{Q}}=\mathcal{E}((-\varphi+\bar{\eta}) \cdot B+\bar{\gamma} * \widetilde{\mu}) \mathrm{d} \mathbb{P}$ and $\mathbb{Q}^{n}:=(1 / n) \widehat{\mathbb{P}}+(1-1 / n) \overline{\mathbb{Q}}$ for $n \in \mathbb{N}$. Then the densities $\mathrm{d} \mathbb{Q}^{n} / \mathrm{d} \mathbb{P}$ of the sequence $\left(\mathbb{Q}^{n}\right)_{n \in \mathbb{N}}$ in $\mathcal{Q}^{\text {ngd }}$ converge to the one of $\overline{\mathbb{Q}}$ in $L^{1}(\mathbb{P})$ for $n \rightarrow \infty$. Consequently, $\pi_{t}^{u}(X) \geq \mathbb{E}_{\overline{\mathbb{Q}}}\left(X \mid \mathcal{F}_{t}\right)$ holds for all $t \leq T$.

Proof. Let $n \in \mathbb{N}$. Clearly $\mathbb{Q}^{n} \approx \mathbb{P}$. Moreover $\mathrm{d} \mathbb{Q}^{n} / \mathrm{d} \mathbb{P}=Z^{n}:=(1 / n) \widehat{Z}+(1-1 / n) \bar{Z}$ with $\widehat{Z}:=\mathrm{d} \widehat{\mathbb{Q}} / \mathrm{d} \mathbb{P}=\mathcal{E}(-\varphi \cdot B)$ and $\bar{Z}:=\mathrm{d} \overline{\mathbb{Q}} / \mathrm{d} \mathbb{P}$. Itô formula then yields $Z^{n}=\mathcal{E}\left(\left(-\varphi+\eta^{n}\right) \cdot B+\gamma^{n} * \widetilde{\mu}\right)$ for $\eta^{n}=\alpha \bar{\eta}$ being predictable and $\gamma^{n}=\alpha \bar{\gamma}$ is $\widetilde{\mathcal{P}}$-measurable with $\alpha=(1-1 / n)\left(\bar{Z} / Z^{n}\right) \in[0,1)$ thanks to $\widehat{Z}>0$. Therefore $\eta^{n} \in \operatorname{Ker} \sigma$ and $\gamma^{n}>-1$ due to $\bar{\gamma} \geq-1$. Hence $\left(\eta^{n}, \gamma^{n}\right) \in C$ and so $\mathbb{Q}^{n}=\mathbb{Q}^{\gamma^{n}, \eta^{n}}$ is in $\mathcal{Q}^{\text {ngd }}$. Convergence of $\mathbb{Q}^{n}$ to $\overline{\mathbb{Q}}$ in $L^{1}(\mathbb{P})$ as $n \rightarrow \infty$ is straightforward by definition of $\mathbb{Q}^{n}$ and this implies $\pi_{t}^{u}(X) \geq \mathbb{E}_{\overline{\mathbb{Q}}}\left(X \mid \mathcal{F}_{t}\right)$ for all $t \leq T$. 
The dynamic good-deal bound $\pi^{u}(X)$ of $X \in L^{\infty}(\mathbb{P})$ is given by the solution to the JBSDE

$$
-\mathrm{d} Y_{t}=\left(\left(-\varphi_{t}+\bar{\eta}_{t}\right) Z_{t}+\int_{E} U_{t}(e) \bar{\gamma}_{t}(e) \zeta_{t}(e) \lambda(\mathrm{d} e)\right) \mathrm{d} t-Z_{t} \mathrm{~d} B_{t}-\int_{E} U_{t}(e) \widetilde{\mu}(\mathrm{d} t, \mathrm{~d} e), \quad t \in[0, T],
$$

for terminal condition $Y_{T}=X$, with $\bar{\gamma}=\bar{\gamma}(Z, U), \bar{\eta}=\bar{\eta}(Z, U)$ given by Lemma 5.10, according to

Theorem 5.12. For $X \in L^{\infty}(\mathbb{P})$, the JBSDE above with $(\bar{\gamma}, \bar{\eta})$ from (5.14) has a unique solution $(Y, Z, U)$ in $\mathcal{S}^{\infty} \times \mathcal{L}^{2}(B) \times \mathcal{L}^{2}(\widetilde{\mu})$. Moreover there exists $\overline{\mathbb{Q}} \ll \mathbb{P}$ in the $L^{1}$-closure of $\mathcal{Q}^{\text {ngd }}$ (cf. Lemma 5.11), with density $\mathrm{d} \overline{\mathbb{Q}} / \mathrm{d} \mathbb{P}=\mathcal{E}((-\varphi+\bar{\eta}) \cdot B+\bar{\gamma} * \widetilde{\mu})$ such that the good-deal bound satisfies

$$
\pi_{t}^{u}(X)=\underset{\mathbb{Q} \in \mathcal{Q}^{\text {ngd }}}{\operatorname{ess} \sup } \mathbb{E}_{\mathbb{Q}}\left(X \mid \mathcal{F}_{t}\right)=Y_{t}=\mathbb{E}_{\overline{\mathbb{Q}}}\left(X \mid \mathcal{F}_{t}\right) \quad \text { for } t \leq T
$$

Proof. Consider the family of BSDE generator functions defined for $(z, u) \in \mathbb{R}^{d} \times L^{2}(\zeta$. $\mathrm{d} \lambda)$ by $f^{(\gamma, \eta)}(\cdot, z, u):=(-\varphi \cdot+\eta \cdot) z+\int_{E} u(e) \gamma \cdot(e) \zeta .(e) \lambda(\mathrm{d} e)$ and $f^{(\gamma, \eta)}(\cdot, z, u):=0$ elsewhere, for $(\gamma, \eta) \in \bar{C}$, where coefficients $\left(\gamma_{t}(\omega),-\varphi_{t}(\omega)+\eta_{t}(\omega)\right)$ of $f^{(\gamma, \eta)}$ are bounded in $L^{2}\left(\lambda_{t}(\omega)\right) \times \mathbb{R}^{d}$ by $K_{f}:=$ $\|K\|_{\infty} \in(0, \infty)$ for all $(\gamma, \eta)$ and $(t, \omega)$. By Lemma 5.10, a classical generator function $f$ for

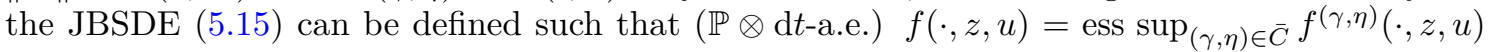
for all $(z, u) \in \mathbb{R}^{d} \times L^{2}(\zeta$. $\mathrm{d} \lambda)$ and $f$ is (a.e.) Lipschitz continuous in $(z, u) \in \mathbb{R}^{d} \times L^{2}\left(\lambda_{t}(\omega)\right)$, with Lipschitz constant $K_{f}$. Indeed, such generator function $f$ can be defined at first (up to a $\mathbb{P} \otimes \mathrm{d} t$-nullset) for countably many $(z, u)$ with $z \in \mathbb{Q}^{d}$ and $u \in\left\{u^{n}, n \in \mathbb{N}\right\}$ dense subset of $L^{2}(\lambda)$ and, noting that $u \zeta_{t}(\omega)^{1 / 2}$ is in $L^{2}(\lambda)$ for $u$ in $L^{2}\left(\lambda_{t}(\omega)\right)$, by Lipschitz-continuous extension for all $(z, u) \in \mathbb{R}^{d} \times L^{2}\left(\lambda_{t}(\omega)\right)$. By setting $f(t, z, u):=0$ elsewhere (for $u \in L^{0}(\mathcal{B}(E), \lambda) \backslash L^{2}\left(\lambda_{t}(\omega)\right)$ ), one can define $f$ as Lipschitz continuous even for $(z, u) \in \mathbb{R}^{d} \times L^{0}(\mathcal{B}(E), \lambda)$.

By classical theory for Lipschitz-JBSDE, equation (5.15) thus has a unique solution $(Y, Z, U)$ in $\mathcal{S}^{2} \times \mathcal{L}^{2}(B) \times \mathcal{L}^{2}(\widetilde{\mu})$ which by boundedness of X satisfies $Y \in \mathcal{S}^{\infty}$ (cf. e.g. [Bec06, Prop.3.2-3.3]). Note that for all $(\gamma, \eta) \in \bar{C}$, clearly $\beta:=-\varphi+\eta$ is bounded and $\int_{E}\left|\gamma_{t}(e)\right|^{2} \zeta_{t}(e) \lambda(\mathrm{d} e)$ is bounded uniformly in $t \leq T$. Hence by Lemma 4.8, the BSDEs with generators $f^{\gamma, \eta}$ also have unique solutions $\left(Y^{\gamma, \eta}, Z^{\gamma, \eta}, U^{\gamma, \eta}\right) \in \mathcal{S}^{\infty} \times \mathcal{L}^{2}(B) \times \mathcal{L}^{2}(\widetilde{\mu})$, which satisfy $Y_{t}^{\gamma, \eta}=\mathbb{E}_{\mathbb{Q}^{\gamma, \eta}}\left(X \mid \mathcal{F}_{t}\right), \mathbb{Q}^{\gamma, \eta}$-a.s., $t \leq T$. Since $f=f^{\bar{\gamma}, \bar{\eta}}$, we also have $Y_{t}=\mathbb{E}_{\overline{\mathbb{Q}}}\left(X \mid \mathcal{F}_{t}\right)$, $\overline{\mathbb{Q}}$-a.s.. By Lemma 5.11 holds $\pi_{t}^{u}(X) \geq \mathbb{E}_{\overline{\mathbb{Q}}}\left(X \mid \overline{\mathcal{F}}_{t}\right)$, $\overline{\mathbb{Q}}$-a.s., for all $t \leq T$. To complete the proof, we show that $\pi_{t}^{u}(X) \leq Y_{t}$. For all $(\gamma, \eta) \in C$ (defining $\left.\mathbb{Q}^{\gamma, \eta} \in \mathcal{Q}^{\text {ngd }}\right)$ we have that $f_{t}\left(Z_{t}, U_{t}\right)=f_{t}^{\bar{\gamma}, \bar{\eta}}\left(Z_{t}, U_{t}\right)$ dominates $f_{t}^{\gamma, \eta}\left(Z_{t}, U_{t}\right)$ for a.e. $t \leq T$. Noting that $f^{\gamma, \eta}$ are Lipschitz in $(z, u)$ with (uniform) Lipschitz constant $K_{f}$ and

$$
f_{t}^{\gamma, \eta}\left(Z_{t}^{\gamma, \eta}, U_{t}\right)-f_{t}^{\gamma, \eta}\left(Z_{t}^{\gamma, \eta}, U_{t}^{\gamma, \eta}\right)=\int_{E} \gamma_{t}(e)\left(U_{t}(e)-U_{t}^{\gamma, \eta}(e)\right) \zeta_{t}(e) \lambda(\mathrm{d} e), \quad t \leq T,
$$

with $\mathcal{E}((-\varphi+\eta) \cdot B+\gamma * \widetilde{\mu})$ being a martingale (see Example 3.3), one can apply comparison as in Proposition 3.1 to get $Y_{t} \geq Y_{t}^{\gamma, \eta}, \mathbb{P}$-a.s., $t \leq T,(\gamma, \eta) \in C$. Hence $Y_{t} \geq \operatorname{ess} \sup _{(\gamma, \eta)} Y_{t}^{\gamma, \eta}=\pi_{t}^{u}(X)$, $t \leq T$, for $(\gamma,-\varphi+\eta)$ ranging over all Girsanov kernels of measures $\mathbb{Q} \in \mathcal{Q}^{\text {ngd }}$.

\section{References}

[AM16] F. Antonelli and C. Mancini. Solutions of BSDEs with jumps and quadratic/locally lipschitz generator. Stochastic Process. Appl., 126(10):3124-3144, 2016.

[Ban15] E. Bandini. Existence and uniqueness for backward stochastic differential equations driven by a random measure, possibly non quasi-left continuous. Electron. Commun. Probab., 20:13 pp., 2015.

[BBP97] G. Barles, R. Buckdahn, and E. Pardoux. BSDE's and integral-partial differential equations. Stochastics, 60:57-83, 1997.

[BC17] E. Bandini and F. Confortola. Optimal control of semi-markov processes with a backward stochastic differential equations approach. Math. Control Signals Systems, 29(1):1, 2017.

[Bec06] D. Becherer. Bounded solutions to backward SDEs with jumps for utility optimization and indifference hedging. Ann. Appl. Probab., 16:2027-2054, 2006. 
[Bec10] D. Becherer. Utility indifference valuation. In R. Cont, editor, Encyclopedia of Quantitative Finance. John Wiley \& Sons, Ltd, Chichester, 2010.

[BEK09] P. Barrieu and N. El Karoui. Pricing, hedging and designing derivatives with risk measures. In R. Carmona, editor, Indifference Pricing, Theory and Applications, pages 77-146. Princeton Univ. Press, 2009

[BEK13] P. Barrieu and N. El Karoui. Monotone stability of quadratic semimartingales with applications to unbounded general quadratic BSDEs. Ann. Probab., 41(3B):1831-1863, 2013.

[BK17a] D. Becherer and K. Kentia. Good deal hedging and valuation under combined uncertainty about drift and volatility. Probab. Uncertain. Quant. Risk, 2(1):13, Dec 2017.

[BK17b] D. Becherer and K. Kentia. Hedging under generalized good-deal bounds and model uncertainty. Math. Meth. Oper. Res., 86(1):171-214, Aug 2017.

[BS06] T. Björk and I. Slinko. Towards a general theory of good-deal bounds. Rev. Financ., 10:221-260, 2006.

[CE10] S.N. Cohen and R.J. Elliott. Comparisons for backward stochastic differential equations on Markov chains and related no-arbitrage conditions. Ann. Appl. Probab., 20:267-311, 2010.

[CE15] S.N. Cohen and R.J. Elliott. Stochastic calculus and applications. Probability and its Applications. Springer, New York, second edition, 2015.

[CF14] F. Confortola and M. Fuhrman. Backward stochastic differential equations associated to jump Markov processes and applications. Stochastic Process. Appl., 124:289-316, 2014.

[CFJ16] F. Confortola, M. Fuhrman, and J. Jacod. Backward stochastic differential equations driven by a marked point process: an elementary approach with an application to optimal control. Ann. Appl. Probab., 26(3):1743-1773, 2016.

[CGMY02] P. Carr, H. Geman, D. Madan, and M. Yor. The fine structure of asset returns: An empirical investigation. Journal of Business, 75(2):305 - 332, 2002.

[CSR00] J. Cochrane and J. Saá Requejo. Beyond arbitrage: Good deal asset price bounds in incomplete markets. J. Polit. Econ., 108:79-119, 2000.

[DM82] C. Dellacherie and P. Meyer. Probabilities and Potenial B: Theory of Martingales. Mathematics Studies. North Holland, Amsterdam, New York, Oxford, 1982.

[DP15] L. Delong and A. Pelsser. Instantaneous mean-variance hedging and Sharpe ratio pricing in a regime-switching financial model. Stoch. Models, 31:67-97, 2015.

[DTE15a] P. Di Tella and H-J. Engelbert. On the predictable representation property of martingales associated with Lévy processes. Stochastics, 87(1):1-15, 2015.

[DTE15b] P. Di Tella and H.-J. Engelbert. The predictable representation property of compensated-covariation stable families of martingales. Theory Probab. Appl., 60:99-130, 2015.

[EFO17] M. Eddahbi, I. Fakhouri, and Y. Ouknine. $\{L\}^{p}(p \geq 2)$-solutions of generalized BSDEs with jumps and monotone generator in a general filtration. Modern Stochastics: Theory and Applications, 4(1):25-63, 2017.

[EMN16] N. El Karoui, A. Matoussi, and A. Ngoupeyou. Quadratic exponential semimartingales and application to BSDEs with jumps. arXiv preprint, arXiv:1603.06191, 2016.

[GL16] C. Geiss and C. Labart. Simulation of BSDEs with jumps by Wiener Chaos expansion. Stochastic Process. Appl., 126(7):2123-2162, 2016.

[GS16a] C. Geiss and A. Steinicke. $L_{2}$-variation of Lévy driven BSDEs with non-smooth terminal conditions. Bernoulli, 22(2):995-1025, 2016.

[GS16b] C. Geiss and A. Steinicke. Malliavin derivative of random functions and applications to Lévy driven BSDEs. Electron. J. Probab., 21:28 pp., 2016.

[GS17] C. Geiss and A. Steinicke. Existence, uniqueness and comparison results for BSDEs with Lévy jumps in an extended monotonic generator setting. To appear in Probab. Uncertain. Quant. Risk, arXiv:1711.01449v2, 2017.

[HIM05] Y. Hu, P. Imkeller, and M. Müller. Utility maximization in incomplete markets. Ann. Appl. Probab., 15:1691-1712, 2005.

[HLT17] Y. Hu, G. Liang, and S. Tang. Exponential utility maximization and indifference valuation with unbounded payoffs. arXiv preprint, arXiv:170\%.00199, 2017. 
[HWY92] S. He, J. Wang, and J. Yan. Semimartingale Theory and Stochastic Calculus. Science Press, CRC Press, New York, 1992.

[JS03] J. Jacod and A. Shiryaev. Limit Theorems for Stochastic Processes. Springer, Berlin, 2003.

[Kaz79] N. Kazamaki. A sufficient condition for the uniform integrability of exponential martingales. Mathematical Reports Toyama University, 2:1 - 11, 1979.

[Kaz94] N. Kazamaki. Continuous Exponential Martingales and BMO. Lecture Notes in Mathematics 1579. Springer, Berlin, 1994.

[Ken15] K. Kentia. Robust aspects of hedging and valuation in incomplete markets and related backward SDE theory. PhD thesis, Humboldt-Universität zu Berlin, 2015. urn:nbn:de:kobv: 11-100237580.

[Kob00] M. Kobylanski. Backward stochastic differential equations and partial differential equations with quadratic growth. Ann. Appl. Probab., 28:558-602, 2000.

[KP16] T. Kruse and A. Popier. BSDEs with monotone generator driven by brownian and poisson noises in a general filtration. Stochastics, 88(4):491-539, 2016.

[KP17] T. Kruse and A. Popier. Lp-solution for BSDEs with jumps in the case $p<2$. Stochastics, 89(8):1201-1227, 2017.

[KTPZ15] N. Kazi-Tani, D. Possamaï, and C. Zhou. Quadratic BSDEs with jumps: a fixed-point approach. Electron. J. Probab., 20(66):1-28, 2015.

[KTPZ16] N. Kazi-Tani, D. Possamaï, and C. Zhou. Quadratic BSDEs with jumps: Related nonlinear expectations. Stoch. Dyn., 16(4):1650012, 32 pp, 2016.

[LM78] D. Lepingle and J. Mémin. Sur l'intégrabilité uniforme des martingales exponentielles. $Z$. Wahrscheinlichkeitstheor. verw. Geb., 42:175-203, 1978.

[LS14] R. J. A. Laeven and M. A. Stadje. Robust portfolio choice and indifference valuation. Math. Oper. Res., 39:1109-1141, 2014.

[MC14] M. Mania and B. Chikvinidze. New proofs of some results on BMO martingales using BSDEs. J. Theoret. Probab., 27:1213-1228, 2014.

[Mor09] M. Morlais. Utility maximization in a jump market model. Stochastics, 81:1-27, 2009.

[Mor10] M. Morlais. A new existence result for quadratic BSDEs with jumps with application to the utility maximization problem. Stochastic Process. Appl., 120:1966-1995, 2010.

[MS05] M. Mania and M. Schweizer. Dynamic exponential utility indifference valuation. Annals Appl. Probab., 15(3):2113-2143, 2005.

[NS00] D. Nualart and W. Schoutens. Chaotic and predictable representations for Lévy processes. Stochastic Process. Appl., 90:109-122, 2000.

[NS01] D. Nualart and W. Schoutens. Backward stochastic differential equations and Feynman-Kac formula for Lévy processes, with applications in finance. Bernoulli, 7(5):761-776, 2001.

[Nut10] M. Nutz. The opportunity process for optimal consumption and investment with power utility. Math. Financ. Econ., 3(3-4):139-159, 2010.

[PP90] E. Pardoux and S. Peng. Adapted solution of a backward stochastic differential equation. System Control Lett., 14:55-61, 1990.

[PPS16] A. Papapantoleon, D. Possamaï, and A. Saplaouras. Existence and uniqueness results for BSDEs with jumps: the whole nine yards. arXiv preprint, arXiv:1607.04214, 2016.

[PS08] P. Protter and K. Shimbo. No arbitrage and general semimartingales. Markov Processes and related Topics: A Festschrift for Thomas G. Kurtz, 4:267-283, 2008.

[QS13] M.-C. Quenez and A. Sulem. BSDEs with jumps, optimization and applications to dynamic risk measures. Stochastic Process. Appl., 123(8):3328-3357, 2013.

[Roc76] R. T. Rockafellar. Integral functionals, normal integrands and measurable selections. In L. Waelbroeck, editor, Nonlinear Operators and Calculus of Variations, Lecture Notes in Mathematics 543, pages 157-207. Springer, Berlin, 1976.

[Roy06] M. Royer. Backward stochastic differential equations with jumps and related non-linear expectations. Stochastic Process. Appl., 116:1358-1376, 2006.

[Tev08] R. Tevzadze. Solvability of backward stochastic differential equations with quadratic growth. Stochastic Process. Appl., 118(3):503-515, 2008. 
[TL94] S. Tang and X. Li. Necessary conditions for optimal control for stochastic systems with random jumps. SIAM J. Control Optim., 32:1447-1475, 1994.

[Yao17] S. Yao. Lp solutions of backward stochastic differential equations with jumps. Stochastic Processes and their Applications, 127(11):3465-3511, 2017. 